Digitized by the Internet Archive in 2010 with funding from UNEP-WCMC, Cambridge 


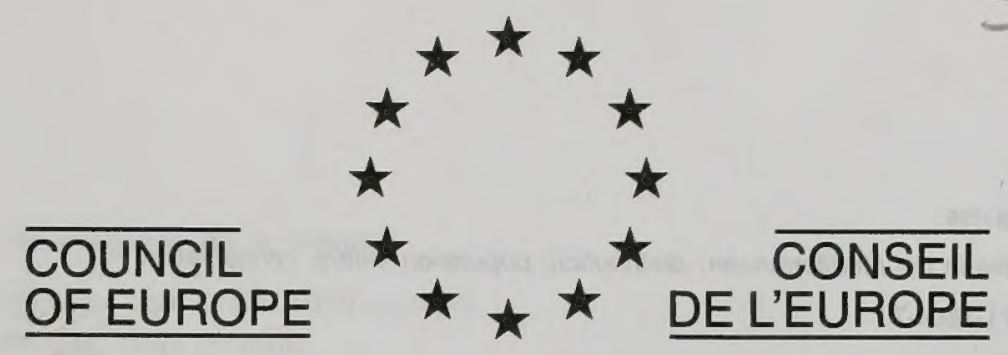

Convention relative à la conservation de la vie sauvage et du milieu naturel de l'Europe

\title{
Les tortues marines en Méditerranée: distribution, populations, protection
}

\author{
Brian Groombridge
}

World Conservation Monitoring Centre

Division de la protection et de la gestion de l'environnement 
Edition anglaise:

Marine turtles in the Mediterranean: distribution, population status, conservation

ISBN 92-871-1864-7

Strasbourg, Conseil de l'Europe, Service de l'édition et de la documentation ISBN 92-871-1871-X

(C) Copyright, Conseil de I'Europe, Strasbourg, 1990 Imprimé en France 


\section{Collection Sauvegarde de la nature}

1. Aménagement des forêts, 1968 (épuisé)

2. Eaux douces, 1968 (épuisé)

3. Animaux menacés, 1969 (épuisé)

4. Le rôle des pouvoirs locaux, 1971 (épuisé)

5. Conservation des sols, 1972 (épuisé)

6. Les régions menacées des Alpes et les mesures de prévention, 1974 (épuisé)

7. Pollution de l'air - Manuel d'expériences, 1975

8. Evolution et conservation des bocages européens, 1975

9. La gestion intégrée du patrimoine faunistique européen, 1975

10. Mammifères menacés en Europe, 1976

11. Les effets de la recréation sur l'écologie des paysages naturels, 1976 (épuisé)

12. Les landes à bruyère de l'Europe occidentale, 1976

13. La dégradation du maquis méditerranéen, 1977 (publication jointe avec l'Unesco) (épuisé)

14. Liste des plantes rares, menacées et endémiques en Europe, 1977 (épuisé)

15. Amphibiens et reptiles menacés en Europe, 1978 (épuisé)

16. Carte (échelle 1:3000 000) de la végétation des Etats membres du Conseil de l'Europe, 1979 (épuisé)

17. Modèle cadre relatif à l'impact sur l'environnement dans l'optique d'un aménagement ou d'une planification intégrée du milieu naturel, 1980

18. Les poissons d'eau douce menacés en Europe, 1980

19. Les tourbières en Europe, 1980

20. Le comportement du public dans les zones protégées, 1981 (épuisé)

21. Les pelouses sèches en Europe, 1981

22. Les forêts alluviales en Europe, 1981 (épuisé)

23. Rhopalocères (papillons diurnes) menacés en Europe, 1981

24. Les oiseaux ayant besoin d'une protection spéciale en Europe, 1981 (épuisé)

25. Inventaire et classification des biocénoses marines benthiques de la Méditerranée, 1982

26. Les fermes urbaines, 1982

27. Liste des plantes rares, menacées et endémiques en Europe (édition 1982), 1983

28. La nature dans la ville, 1983

29. La végétation de l'arc alpin, 1983

30. La végétation halophile en Europe (prés salés), 1984

31. Les zones marines protégées, 1985

32. La végétation des dunes et bordures des plages européennes, 1985

33. Les répercussions écologiques de la construction et de l'exploitation de pistes de ski, 1986

34. Une pédagogie de l'environnement pour le milieu agricole - Expériences européennes, 1987

35. Invertébrés ayant besoin d'une protection spéciale en Europe, 1987

36. Développement de la faune et de la flore en territoire urbain, 1987

37. Conservation des biocénoses marines benthiques de la mer du Nord et de la Baltique, 1987

38. Protection des libellules (Odonates) et de leurs biotopes, 1988

39. Problèmes de conservation du sol, 1988

40. Textes adoptés par le Conseil de l'Europe dans le domaine de la conservation de la vie sauvage et du milieu naturel de l'Europe, 1989

41. Biologie, statut et conservation du Phoque moine (Monachus monachus), 1989

42. Les invertébrés saproxyliques et leur protection, 1989 
42. Les invertébrés saproxyliques et leur protection, 1989

43. Causes possibles du dépérissement des forêts et programmes de recherche en Europe, 1989

44. L'importance biologique et la conservation des hyménoptères en Europe, 1990

45. Statut, besoins de conservation et réintroduction du Lynx (Lynx lynx) en Europe, 1990

46. Protection des poissons d'eau douce menacés en Europe, 1990

47. Statut et conservation du Loup (Canis lupus) dans les Etats membres du Conseil de l'Europe, 1990

48. Les tortues marines en méditerranée: distribution, populations, protection, 1990

\section{Collection Rencontres environnement}

1. La formation environnementale des milieux agricoles, 1987

2. Parcs, plans et population - Les zones protégées et le développement socio-économique, 1987

3. Atelier sur la situation et la protection des forêts anciennes naturelles et semi-naturelles en Europe, 1987

4. Pour une nouvelle gestion de l'environnement dans l'espace rural, 1987

5. Formation des gestionnaires de zones protégées européennes et africaines, 1988

6. Situation et protection de l'Ours brun (Ursus arctos) en Europe, 1988

7. Quel avenir pour notre nature?, 1989

8. Colloque Patrimoine génétique «la conservation des espèces sauvages progénitrices des plantes cultivés», 1990

9. Séminaire sur les musées de la nature: outils pour la connaissance, la valorisation et la préservation du patrimoine naturel européen,1990

\section{Série Aménagement et gestion}

1. La haie, 1987

2. Agriculture et vie sauvage, 1989 


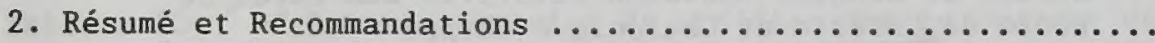

2.1. Résumé

2.2. Recommandations

3. Plages de ponte et distribution des espèces

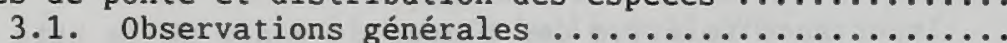

3.2. Albanie

3.3. Algérie

3.4. Chypre

3.5 Egypte

3.6.

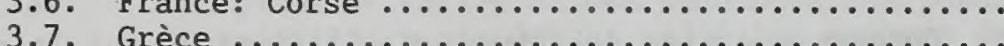

3.8. Israël

3.9. Italie

3.10. Liban

3.11. Libye

3.12. Malte

3.13. Monaco

3.14. Maroc

3.15. Espagne

3.16. Syrie

3.17. Tunisie

3.18. Turquie

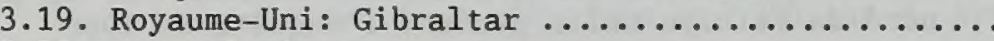

3.20. Yougoslavie

4. Populations reproductrices de Méditerranée

4.1. L'origine des populations reproductrices de

Méditerranée

4.2. Dimensions et tendances des populations

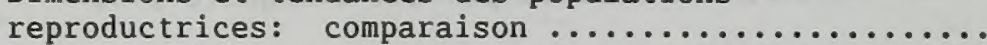

4.3. Menaces qui pèsent sur les populations reproductrices

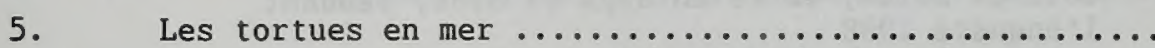

5.1. Va-et-vient entre Méditerranée et Atlantique ...

5.2. Mouvements des tortues en Méditerranée .........

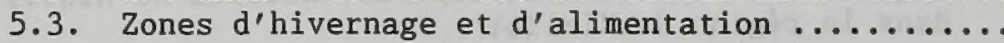

5.4. Les tortures en Méditerranée orientale.........

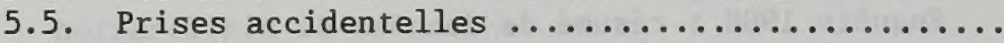

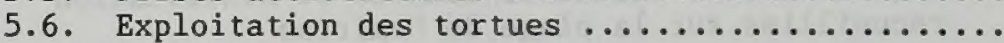

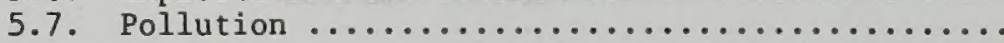

6. Espèces non reproductrices et occasionnelles

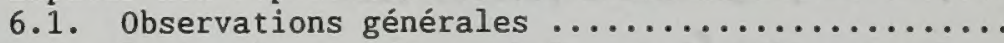

6.2. Tortue à cuir (Dermochelys coriacea)

6.3 Tortue à écailles (Eretmochely imbricata)

6.4. Tortue de Kemp (Lepidochelys kempii) ...........

6.5. Tortue olivâtre (Lepidochelys olivacea)

7. Accords internationaux

8. Remerciements

9. Références 


\section{LISTE DES TABLEAUX}

Tableau 1. Résumé de données choisies relatives aux lieux de ponte de Caretta et Chelonia à Chypre

Tableau 2. Résumé de données choisies relatives aux lieux de ponte de Caretta en Grèce

Tableau 3. Importance relative des plages de ponte de la baie de Laganas, à Zante

Tableau 4. Résumé de données choisies relatives aux plages de ponte de Caretta dans la baie de Laganas, à Zante

Tableau 5. Données choisies relatives au nidification sur la plage de Potamakia, à Cephalonia

Tableau 6. Données sur l'émergence et la nidification des tortues sur la plage de Dalyan, 4 juin-5 juillet 1987

Tableau 7. Les nidifications de Caretta à Dalyan, 1987-1989

Tableau 8. Résumé des données de nidification rassemblées sur la plage de Sarigerme-Dalaman au cours de l'enquête de 1988

Tableau 9. Résumé de données de nidification réunies sur la plage nord de Fethiye-Calis pendant l'enquête de 1988

Tableau 10. Résumé de données de nidification réunies sur la plage de Patara dans le cadre de l'enquête de 1988

Tableau 11. Résumé de données de nidification recueillies sur la plage de Finike-Kumluca pendant l'enquête 1988

Tableau 12. Résumé de données choisies de nidification réunies sur la côte de Belek, entre Antalya et Side, pendant l'enquête 1988

Tableau 13. Enquête 1988 : résumé de données choisies de nidification dans la région de Kizilot

Tableau 14. Enquête 1988 : résumé de données de nidification recueillies sur la plage de Demirtas

Tableau 15. Enquête 1988 : résumé de données de nidification pour la plage de Gazipasa Ciftlik

Tableau 16. Enquête 1988 : résumé de données de nidification au delta de Göksü

Tableau 17. Enquête 1988 : résumé de données choisies de nidification sur la plage de Kazanli

Tableau 18. Enquête 1988 : résumé de données choisies de nidification sur la plage d'Akyatan 
Tableau 19. Enquête 1988 : résumé de données choisies de nidification sur la plage de Samandagi

Tableau 20. Données choisies de nidification au sud-est de la Turquie (delta de Cukurova et golfe d'Iskenderun), 1988

Tableau 21. Résumé de données choisies de nidification au sud-est de la Turquie (delta de Cukurova et golfe d'Iskenderun), entre le 25 mai et le 16 août 1988

Tableau 22. Plages connues de nidification des tortues marines et nombre de reproductrices en Turquie

Tableau 23. Aperçu de la taille et des tendances des populations reproductrices passées et actuelles connues de caouannes (Caretta Caretta) en Méditerranée

Tableau 24. Aperçu de la taille et des tendances des populations reproductrices passées et actuelles connues de chélonées franches (Chelonia mydas) en Méditerranée

Tableau 25. Estimation du nombre total de femelles nichant chaque année en Méditerranée

Tableau 26. Estimation provisoire du nombre de tortues tuées par la pêche en Méditerranée occidentale, sur la base d'entretiens effectués en 1978 par R. Argano

Tableau 27. Aperçu de la prise annuelle de tortues en Médi terranée

Tableau 28. Historique résumé de la prise commerciale de tortues en Méditerranée, sur les principaux sites connus

Tableau 29. Principaux accords internationaux relatifs aux tortues marines de Méditerranée, et pays adhérents 


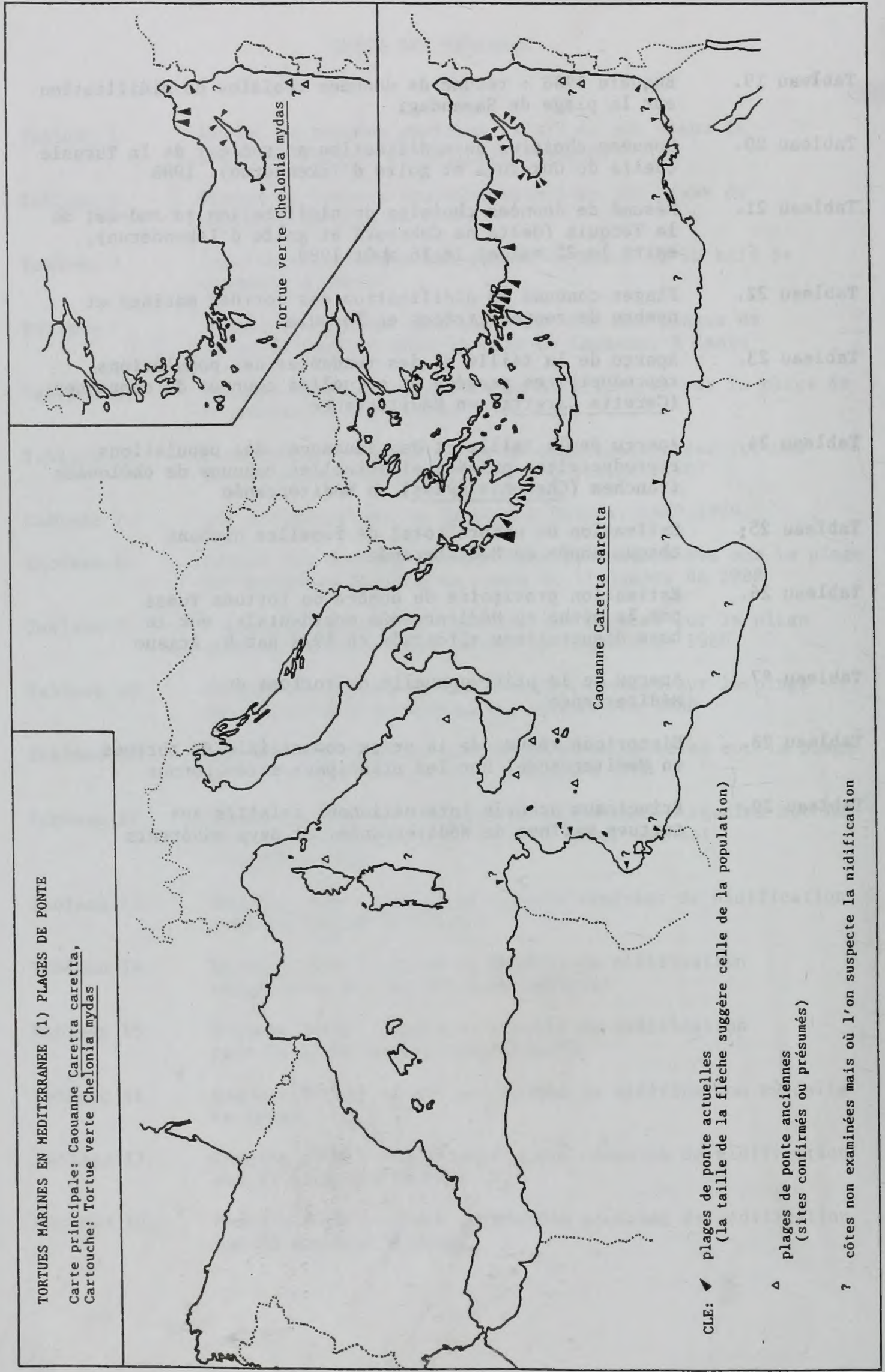


$-9-$

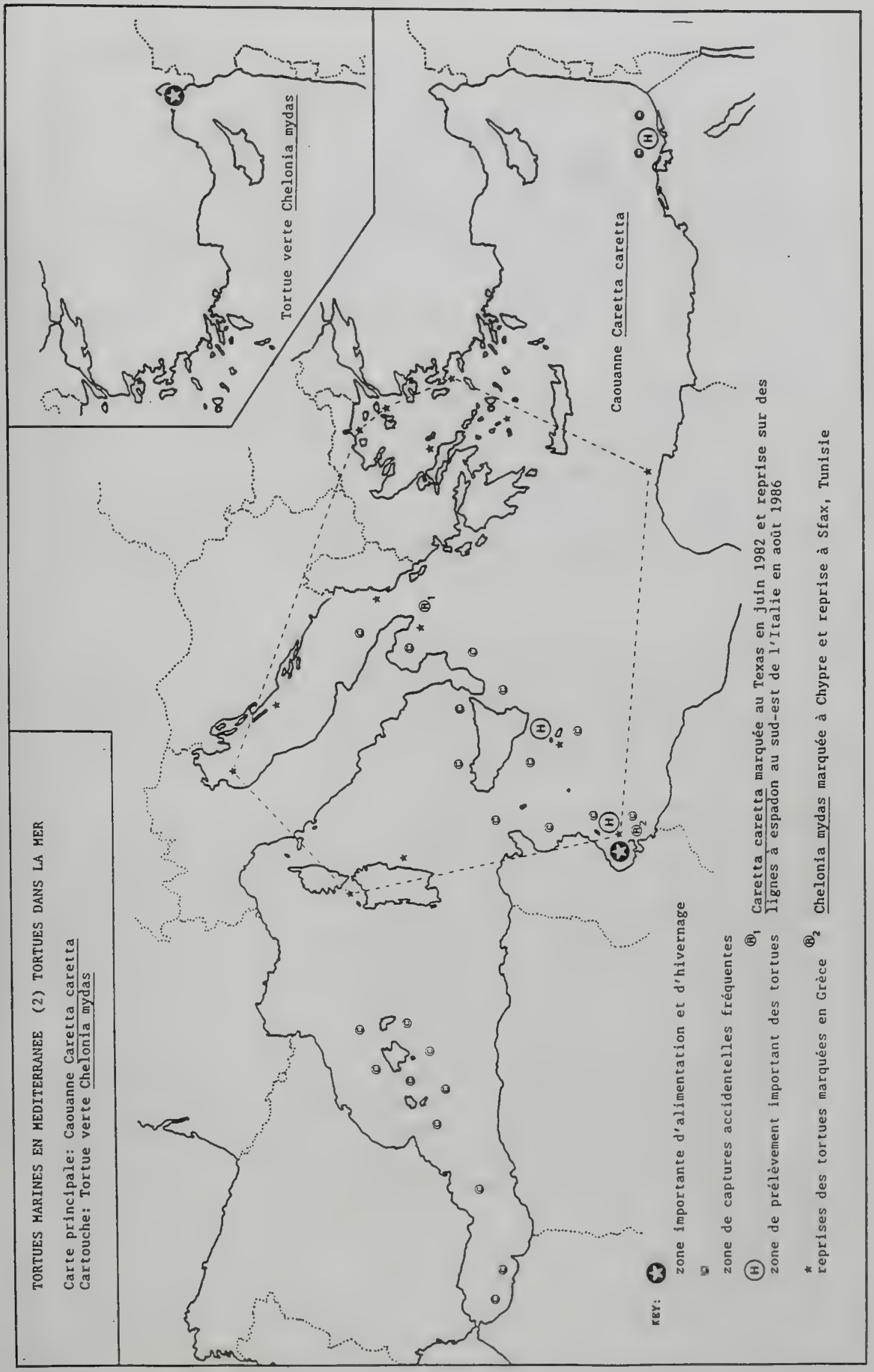





\section{INTRODUCTION}

Le présent rapport, qui couvre la protection et la distribution géographique des tortues marines, leurs plages de ponte et leurs zones d'alimentation en Héditerranée, a été préparé en vertu du contrat $n^{\circ} 32.88$ de la Division de la protection et de la gestion de l'environnement, organe du Secrétariat Général du Conseil de l'Europe. La première version contenait l'information dont on pouvait disposer à la date du 5 décembre 1988. On a révisé cette version en mars 1990, afin d'y incorporer diverses observations et de nouveaux renseignements recueillis sur le terrain au cours de la saison 1988.

Il s'agit essentiellement d'un document récapitulatif qui rassemble et résume $l^{\prime}$ information en vue d'assurer une base de prévision.

On accorde une attention particulière à deux espèces (Caretta caretta et Chelonia mydas) qui nichent régulièrement et en grand nombre en Méditerranée. On peut mener des recherches lentables et prendre des mesures de protection réalistes pour ces espèces, ce qui n'est pas le cas pour les espèces rares, occasionnelles ou accidentelles qui pénètrent dans la région mais généralement sans y nicher. Ce groupe comprend Dermochelys coriacea (qui peut nicher de façon irrégulière), Eretmochelys imbricata et Lepidochelys kempii. Les cinq espèces citées sont reconnues comme menacées dans le monde entier, la caouanne étant classée "vulnérable", et les autres, "en péril" (UICN, 1988).

L'étude ne recouvre que la Méditerranée, la mer Noire étant exclue. On $n^{\prime} y$ aborde pas les questions générales d'écologie, de biologie des populations et de protection des tortues marines: on trouve beaucoup d'information sur ces sujets, avec des bibliographies, dans Bjorndal (1982), Mrosovsky (1983), Groombridge (1982), et Groombridge et Luxmoore (1989). Laurent (1989b) fournit d'autres données sur les tortues marines de la Méditerranée.

La partie principale du rapport consiste en exposés relatifs aux divers pays. Chaque exposé comprend une information donnée sous les rubriques suivantes: observations générales, plages de ponte et nombres, tendances des populations, les tortues en mer, les menaces, les mesures de protection, divers. Lorsque les données manquent pour une rubrique, celle-ci est supprimée. Autant que possible, on a doublé la rubrique relative aux plages de ponte et aux nombres, afin de permettre un traitement séparé de la caouanne et de la chélonée franche. Quelques sections plus longues se divisent en sous-rubriques qui peuvent différer d'un pays à l'autre.

Malgré un effort pour offrir des données numériques sur empreintes et nids, et des estimations du nombre de femelles que l'on peut en déduire, on ne doit pas oublier le caractère fragmentaire desdites données. En particulier, les renseignements relatifs à un site ne peuvent guère être strictement comparables avec ceux qui intéressent un autre site et ont été réunis par des enquêteurs différents. Pour certains secteurs des côtes méditerranéennes, nous ne disposons même pas de données imprécises, de sorte que plusieurs des conclusions auxquelles nous parvenons ici devront être modifiées à la lumière d'études futures. 


\subsection{RESUME}

2.1.1. Deux espèces de tortues marines nichent régulièrement en Méditerranée: la caouanne (Caretta caretta) et la chélonée franche ou tortue verte (Chelonia mydas), la première étant la plus commune et la plus répandue. Trois autres espèces sont présentes mais sans nicher: la tortue cuir (Dermochelys coriacea) est signalée assez régulièrement et niche peut-être à l'occasion; la tortue à écaille (Eretmochelys imbricata), et Lepidochelys kempii qui sont extrêmement rares en Méditerranée et semblent être des migrantes occasionnelles. Aucune enquête n'a été effectuée quant au nidification éventuel de tortues sur les côtes de Libye, d'Egypte et de Syrie, de sorte que l'information disponible sur les plages de ponte est incomplète et qu'il reste possible de découvrir des plages de ponte importantes, notamment sur les rives d'Afrique du Nord. Une bonne partie des côtes d'Afrique du nord et de la Syrie n'a pas été examinée, de sorte que l'information sur les plages de ponte est incomplète et que la découverte d'autres plages importantes à cet égard reste possible.

\subsubsection{Il existe des plages de ponte de la caouanne en Grèce} (notamment à Zante, mais aussi dans le Péloponnèse et ailleurs), en Turquie (côte méridionale), à Chypre et en Libye. On a signalé des pontes, mais en petit nombre, en Tunisie, et il y en aurait également en Algérie et au Maroc, mais nous ne disposons d'aucune donnée à ce sujet. On trouve des couvées occasionnelles et isolées à Lampedusa (Italie), en Sicile, en Israël, et peut-être en Sardaigne; il semble y avoir eu jadis des pontes plus fréquentes en ces divers lieux (très certainement en Israël), et il y en eut aussi en Corse et à Malte, où $l^{\prime}$ on $n^{\prime}$ en signale pas actuellement. Sur la foi des données que nous avons sur les plages de ponte connues, il y a peut-être une moyenne de 2000 femelles caouannes qui nichent annuellement en Méditerranée, la plupart se partageant entre la Grèce et la Turquie.

\subsubsection{La population de Caretta nichant dans la baie de Laganas, à}

Zante (Grèce) est de loin la plus nombreuse colonie reproductrice connue en Méditerranée: elle a accueilli entre 300 et 700 femelles reproductrices, ces dernières saisons. Mais les lieux sont aujourd'hui sérieusement modifiés et troublés par le développement touristique. Les plages de ponte de la partie occidentale de la baie ne sont plus adaptées aujourd'hui en raison du bruit, de l'éclairage, de la circulation des véhicules et en général d'une activité humaine intense, en sorte que le nidification se concentre sur la plage la plus isolée, où la densité est grande. Des décisions récentes de planification et d'affectation des sols, qui auraient dû en principe protéger la population de tortues, n'ont pas été appliquées. Bien que des données récentes sur les nidifications $n^{\prime}$ indiquent pas nettement une diminution constante des nombres, la population est gravement menacée par la modification des plages de ponte et des eaux adjacentes et, à défaut de mesures urgentes, la poursuite de la dégradation semble inévitable.

2.1.4. Les nidifications de caouannes sont très fréquents sur la côte méridionale de la Turquie. Presque tous les lieux de ponte turcs sont menacés par le développement touristique actuel ou projeté; on réexamine actuellement les projets en fonction du besoin de protection des tortues. Dalyan-Iztuzu, qui fait partie de la dizaine de plages de ponte d'importance analogue, a probablement accueilli une centaine de femelles reproductrices en 1987 . Des projets de grand accroissement du développement touristique, à Dalyan, ont été modifiés en 1988. Le 
gouvernement turc a déclaré Dalyan-Iztuzu et Fethiye "zones de protection spéciale", en 1988, et a étendu la même protection, en 1990, à d'importantes plages de Caretta à Patara et au delta de Göksü (en plus d'Ekinchik, point de nidification mineur).

2.1.5. La population de caouannes (C. caretta) nichant à Chypre est relativement faible et dispersée, avec des petites colonies réparties sur plusieurs sites. La plupart des plages du sud de l'île sont aujourd'hui largement bâties. Il reste d'importantes plages dans le secteur de Lara et autour de Polis. Une législation récente a encore renforcé la protection des tortues à Lara, et ce secteur fait partie de la zone récemment érigée en "Parc national d'Akamas". On a entrepris à Lara un effort de protection en y transférant de nombreuses couvées, afin de réduire la mortalité naturelle par prédation ou inondation. Au nord de l'ille, de nombreux sites sont peu perturbés, mais le tourisme s'y développe rapidement, et il existe déjà des projets de construction autour de plages d'une certaine importance; trois des territoires récemment désignés comme secteurs protégés comprennent des plages de ponte.

2.1.6. La tortue verte, Chelonia mydas, autant que nous sachions, ne niche que dans l'extrême sud-est turc (principalement à Kazanli et Akyatan, dans le delta de Cukurova), ainsi qu'à Chypre (on a trouvé un nid isolé en Israël). Il pourrait avoir des nidifications sur les côtes égyptiennes. Il se faisait une pêche commerciale intense de l'espèce au large de la côte septentrionale de ce qui est devenu Israël (entre les deux guerres) et au sud-est de la Turquie (après la deuxième guerre mondiale). Ces deux pêcheries ont capturé des milliers de chélonées franches; la quasi extinction de la population israélienne de l'espèce, et apparemment aussi de la population turque, sont attribuées pour une large part à une surexploitation, aggravée plus récemment par la construction. On ne connaît pas précisément le nombre de reproductrices en Turquie mais des données préliminaires récentes suggèrent qu'environ 300-350 femelles ont niché en 1988, ce qui est très nettement inférieur au chiffre de 1000 ou plus avancé en 1979-1982. Cette population de C. mydas, qui est de loin la plus grande de la Méditerranée, doit être considérée comme extrêmement menacée. De même, on ne connaît qu'imparfaitement le nombre de reproductrices de Chypre, mais on a calculé récemment qu'environ 25 femelles pouvaient nicher chaque année dans la zone de Lara, et peut-être un nombre semblable sur les plages du nord de l'île.

2.1.7. Partout les populations reproductrices pour lesquelles on possède des données sont soit gravement menacées - le plus souvent et le plus évidemment par le tourisme international et la construction sur les côtes -, soit réputées en déclin depuis le début de ce siècle. Presque toutes les grandes populations reproductrices connues, notamment dans la baie de Laganas, au sud de Zante, et le long de la côte méridionale turque, sont plus ou moins gravement menacées par le développement touristique. De rares populations locales, par exemple les chélonées nichant près de Dipkarpaz au nord de Chypre, ou les caouannes dans la baie de Kiparissia, dans le Péloponnèse, semblent relativement peu troublées sur leur plage de ponte.

2.1.8. Aucune des populations reproductrices de tortues marines de la Méditerranée n'est grande selon les normes mondiales. Dans l'ensemble, l'état de conservation des populations de tortues marines qui nichent dans le bassin méditerranéen va de mauvais, dans le cas de la caouenne, à très mauvais, dans le cas de la chelonée franche. Leurs perspectives de survie à long terme ne sont pas favorables, à moins que ne soient prises rapidement des mesures de protection et de gestion rigoureuses. 
2.1.9. Il y a peut-être quelque 50.000 prises accidentelles chaque année; un nombre important de tortues sont prises plus d'une fois. La mortalité qui en découle est incertaine, mais elle pourrait intéresser plus de 10.000 individus, ce qui équivaut à plusieurs fois

l'estimation du contingent annuel de nidifications en Méditerranée. On dispose jusqu'ici de peu de données sur la Méditerranée orientale, mais la plus grande partie, de loin, des prises incidentes connues se produisent dans les pêcheries d'espadon à l'aplet, autour des Iles Baléares et au sud de l'Italie. Selon la fréquence avec laquelle les tortues de l'Atlantique pénètrent en Méditerranée, une bonne part des victimes des prises incidentes peut consister en tortues d'origine autre que méditerranéenne.

2.1.10. Les tortues de la Méditerranée souffrent également de la pollution, mais il s'agit ici d'un phénomène que l'on a encore peu étudié. Certaines plages, notamment à Chypre et dans le sud-est de la Turquie, sont très polluées par des déchets plastiques flottants et du goudron. On a trouvé de nombreuses caouannes, autour de Malte et dans les eaux voisines, avec du goudron sur la tête, dans la cavité buccale et dans l'intestin. Un grand nombre de caouennes mortes échouées sur les plages du sud de l'Italie seraient victimes de la pollution. On sait peu de chose de la fréquence et des effets, pour les tortues, d'autres polluants, tels que les métaux lourds et les résidus de pesticides. On pense que les tortues du sud-est de la Turquie sont très exposées aux métaux lourds et au fort ruissellement de pesticides provenant de la culture intensive des plaines du sud d'Adana.

2.1.11. Les tortues marines font $l^{\prime}$ objet $d^{\prime}$ une importante exploitation à des fins alimentaires ou médicinales en Tunisie, où la prise annuelle est estimée à 5.000 animaux (principalement Caretta); à une échelle plus modeste dans les eaux autour Malte, la Sicile et la Sardaigne, et dans une mesure inconnue en Egypte. Il semble que toutes les tortues soient prises à l'occasion de la pêche en mer, et non sur les plages de ponte. L'exploitation actuelle en Méditerranée centrale doit contribuer beaucoup à la gravité des menaces qui pèsent sur ces espèces dans la région. Une diminution et un contrôle des prises de tortues sont essentiels. Autant que nous sachions, l'utilisation de la tortue ne revêt une importance socio-économique particulière qu'en Tunisie, où la chair de cet animal est meilleur marché que le poisson, et en Egypte.

\subsubsection{Les tortues marines qui nichent dans l'Atlantique sont} certainement capables de pénétrer en Méditerranée par le détroit de Gibraltar, et l'on soupçonne qu'une forte proportion des caouannes que l'on voit en mer autour des Baléares et en Méditerranée centrale proviennent de l'Atlantique. Une caouanne d'un an lâchée au Texas a été recapturée dans les eaux italiennes quatre ans plus tard. On ignore tout à fait si les tortues migrent dans l'autre direction et sortent en nombre de la Méditerranée; le régime des courants dans le détroit et la configuration d'ensemble du bassin pourraient rendre une telle migration difficile.

2.1.13. Nous $\mathrm{n}^{\prime}$ avons qu'une information incomplète sur la distribution des tortues en dehors de la saison de ponte; la plupart des observations sont le fait de pêcheurs, généralement à la recherche de poisson ou de crustacés, de sorte que les données reflètent autant la distribution des observateurs que celle des tortues. Le golfe de Gabès, vers le sud de la Tunisie, est certainement un important site d'alimentation et d'hivernation de la tortue. La plupart des 
recaptures de caouannes baguées en Grèce ont lieu à Gabès, généralement pendant les mois d'hiver. Le golfe de Mersin et celui d'Iskenderun, au sud-est de la Turquie, constituent des zones d'alimentation et d'hivernage pour Chelonia. On y trouve toute I'année des chélonées franches, immatures ou adultes. On a trouvé des zones d'hibernation ou d'hivernage régulier de caouannes en Adriatique centrale et septentrionale.

\subsection{RECOMMANDATIONS}

2.2.1. Il faut assurer de toute urgence une protection suffisante aux principales plages de ponte et aux zones marines proches utilisées par les tortues reproductrices.

Si ces lieux ne sont pas convenablement protégés, la rapide expansion du tourisme international et d'autres aménagements côtiers abaisseront probablement les nombres de nidifications et d'éclosions au-dessous de la limite tolérable.

Les éléments essentiels de la protection de la plupart des principales plages de ponte sont indiqués dans les recommandations faites aux gouvernements de Grèce et de Turquie par le Comité permanent de la Convention relative à la conservation de la vie sauvage et du milieu naturel de l'Europe. La recommandation générale portant sur la protection des tortues et de leurs habitats comprend des recommandations pour la protection des plages, la limitation des aménagements, les travaux de recherche, la coordination et l'éducation. Ces recommandations doivent être mises en oeuvre le plus largement et le plus rapidement possible, et il importe de contrôler et de signaler les progrès accomplis à cet égard. Les plages de ponte qui ont le plus besoin de protection sont:

1. Les plages à caouanne de la baie de Laganas, à Zante;

2. Les grandes plages de ponte de Caretta au sud de la Turquie, y compris, entre autres, Dalyan et Patara (on ne connaît pas précisément l'importance relative des dix grands lieux de ponte reconnus par Baran et Kasparek, 1989a).

3. Les grandes plages de ponte de Chelonia au sud-est de la Turquie, et notamment celles de Karatas et Akyatan, dans le delta de Cukurova, au sud d'Adana.

4. Les plages à chélonée franche de Chypre, y compris celles de la région de Lara et de la péninsule de Karpas, en particulier au nord-ouest de Dipkarpaz.

$\mathrm{Vu}$ les indices de déclin massif de la population et le nombre vestigial de tortues qui nichent actuellement, il faut accorder une protection tout à fait prioritaire aux plages de ponte de Chelonia, au sud-est de la Turquie et à Chypre.

2.2.2. Des mesures s'imposent également pour la protection des tortues en dehors de leur phase de nidification, et notamment sur les lieux d'alimentation, où elles sont menacées par les prises accidentelles, la pêche délibérée et la pollution. 
Sauf, semble-t-il, sur les côtes d'Egypte, de Malte et d'Italie méridionale, il y a relativement peu de prises de tortues marines en Méditerranée. La plupart des prises se font à l'occasion d'autres activités de pêche; en Tunisie, on exploite les tortues prises accidentellement au chalut. On ne sait dans quelle mesure les victimes sont les seules tortues nichant en Méditerranée, ou sont aussi des migrantes venues de l'extérieur. Les actions prioritaires seraient les suivantes:

1. Faire une étude plus poussée des moyens techniques et pédagogiques pour réduire les prises incidentes de tortues et la mortalité concomitante;

2. Préciser la proportion de prises accidentelles se soldant par la mort des animaux;

3. Chercher dans quelle mesure les victimes de prises incidentes sont des tortues nichant hors de la Méditerranée;

4. De façon plus générale, étudier l'importance et les modalités des allées et venues de tortues entre Méditérranée et Atlantique;

5. Eliminer, autant que possible, les prises délibérées de tortues. Il faut trouver des moyens pour réduire la demande de chair de tortue chez les populations côtières, notamment en Tunisie, où elle constitue une importante ressource alimentaire.

6. Mieux reconnaître les sites préférés d'alimentation et d'hivernage, à la fois pour améliorer les connaissances scientifiques et pour permettre de réduire les menaces contre les tortues en dehors de la saison de ponte.

7. Etudier dans quelle mesure les populations de tortues sont affectées par la pollution, y compris celle engendrée par les résidus d'hydrocarbure en général et la pollution issue de l'agrochimie et des métaux lourds signalée dans les eaux au large de l'Italie du sud et de la Turquie du sud-est et rechercher les moyens de réduire cette pollution.

2.2.3. L'information sur les populations de tortues, et notamment sur les éventuelles colonies reproductrices, est très rare pour la plus grande partie des côtes de Libye, d'Egypte et de Syrie. Ces régions peuvent faire l'objet $d^{\prime}$ une étude qui permettrait une évaluation plus complète des conditions générales que trouvent les populations de tortues marines en Méditérranée.

2.2.4. Il faut déployer tous les efforts possibles pour assurer des conditions naturelles sur les plages de ponte. Il ne faut entreprendre qu'avec précaution les intrusions ou des manipulations telles que l'incubation artificielle ou certains types de recherche en biologie du nidification. Il ne faut encourager l'incubation artificielle que $s^{\prime}$ il est prouvé que celle-ci peut réduire les pertes subies en situation naturelle, et non en tant que remède à la perte ou à la perturbation de plages de ponte, lorsque ces maux peuvent être évités. 
2.2.5. Les efforts de recherche et de conservation des tortues de la Méditérranée sont actuellement mal coordonnés, ce qui se traduit par un défaut d'efficacité dans l'emploi du temps et des ressources financières. Ces activités devraient être supervisées par un centre ou une organisation unifiée, plutôt que par de multiples organismes, apparemment concurrents, comme $c^{\prime}$ est le cas actuellement. La protection des tortues de la Méditerranée est un problème international qui appelle des solutions et un financement internationaux. Il faut coordonner plus efficacement la recherche et la conservation actuelles et futures, et améliorer collecte et diffusion de l'information pertinente. 


\section{PLAGBS DB PONTE ET DISTRIBUTION DES ESPECES}

\subsection{OBSERVATIONS GÉNERRALES}

On connaît deux espèces de tortues marines nichant en Méditerranée : la caouanne (Caretta caretta), et la chélonée franche (Chelonia mydas).

Ces deux espèces se trouvent partout dans le monde, la chelonée étant strictement tropicale ou quasi tropicale, la caouanne se rencontrant plus fréquemment à des latitudes supérieures, dans les régions subtropicales et tempérées. Cette apparente diversité des préférences en matière de température se reflète assez dans la distribution des deux espèces en Méditérranée. Le long de la côte turque, par exemple (Geldiay et coll., 1982), la limite orientale de nidification de $\mathrm{C}$. caretta correspond approximativement à l'isotherme

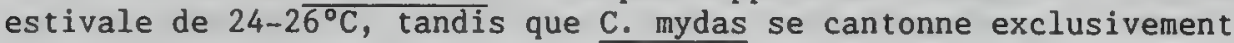
plus à l'est, où les températures estivales sont de $28^{\circ} \mathrm{C}$ ou davantage, de sorte que tout les nidifications de chelonées franches de la Méditerranée se situent à l'extême est. La caouanne, en revanche, est la seule reproductrice de Méditerranée centrale, et le nidification devient $r$ are

ou est tout à fait absent vers l'ouest, où les températures moyennes risquent d'être trop basses. Les caouannes quittent les eaux du nord-ouest de la Méditerranée, celles de Corse par exemple, lorsque les températures s'abaissent en automne et en hiver. On ne sait cependant dans quelle mesure l'actuelle distribution du nidification résulte des modifications apportées par l'homme à l'habitat des tortues. Nous donnons quelques précisions supplémentaires sur le nidification dans les exposés par pays que l'on trouve ci-dessous.

\subsection{ALBANIE}

On ne dispose que de peu d'information récente. Argano et Cocco (1988) rapportent qu'une Caretta baguée au large de I'Italie méridionale a été recapturée au large de l'Albanie, tout comme le fut une femelle baguée lors d'un nidification en Grèce (Margaritoulis, 1988a). On rencontre sans doute des tortues régulièrement dans les eaux albanaises. Frommold (1962) signale avoir vu à l'Institut zoologique de l'Université de Tirana un specimen de Caretta et un autre de Dermochelys, tous deux capturés dans les eaux albanaises. Il ne semble pas probable qu'il y ait des nidifications importants dans la région. Personne n'a signalé de nidification dans le passé.

\subsection{ALGERIE}

\subsubsection{Plages de ponte et nombres}

Bien que toutes les plages n'aient pas été examinées au cours de la saison de nidification probable, il ne semble pas y avoir de nidificati ons

importants de tortues marines sur les rivages algériens; on a des indices d'éclosions occasionnelles et très isolées, mais aucun de l'existence passée de colonies reproductrices. Bien qu'il existe des plages sablonneuses convenables, la température relativement basse de l'eau et le régime actuel de la Méditerranée occidentale empêchent vraisemblablement les nidifications de tortues, comme c'est peut-être le cas aussi en Espagne et au Maroc. 
Après un travail préliminaire sur le terrain en 1978, Argano a indiqué que la côte algérienne ne présentait pas d'intérêt particulier pour ce qui est du nidification, malgré la présence de grandes plages (Argano, 1979). Selon la Direction des Parcs et de la Protection de la Faune (in litt., 10 septembre 1986), aucune Chelonia mydas ni Eretmochelys imbricata $n^{\prime} a$ été signalée en Algérie.

Laurent (1989a) a étudié un échantillon de plages, s'est entretenu avec des pêcheurs et d'autres personnes, et a examiné une documentation remontant jusqu'au milieu du dix-neuvième siècle. Il n'a enregistré aucun indice de nidification en juin et juillet. Seules trois des nombreuses personnes interrogées se rappelaient avoir vu des empreintes présumées de tortues (deux avaient vu des empreintes uniques, un autre, un groupe de quatre à Andalouses, en Algérie occidentale), mais ces témoignages ne s'accompagnent d'aucun document écrit. Laurent $n^{\prime} a$ trouvé aucun signalement de première main de nidifications dans la documentation ancienne ou récente, ni aucun témoignage écrit ; par exemple, un rapport de Doumergue (1899) ne se réfère qu'à des Carreta non adultes (longueur maximale : $70 \mathrm{~cm}$ ) trouvées en mer, et un ouvrage de Fournet (1853) parle explicitement de l'absence de nidifications de tortues en Algérie.

\subsubsection{Les tortues en mer}

Les sources historiques citées par Laurent (1989a) indiquent que les Caretta étaient abondantes au milieu du dix-neuvième siècle et au début du vingtième. Un nombre substantiel de Caretta sont prises accidentellement par des pêcheurs (Laurent, 1989a), ce qui indique qu'il existe encore des populations importantes au large des côtes algériennes. Selon Chalabi (in litt., 19 septembre 1988), des pêcheurs captures parfois Dermochelys coriacea accidentellement.

Un troupeau de plusieurs centaines de caouannes, d'un poids estimé entre 20 et 40 kilos chacune, a été aperçu par l'équipage du bac "Tassili", à une centaine de kilomètres d'0ran, sur le trajet entre l'Espagne (Alicante) et l'Algérie (Lanteri, 1982). Le fait a été observé vers $16 \mathrm{~h}$, le 17 février 1980, alors que les tortues se dirigeaient vers l'est, à une dizaine de mètres de distance l'une de l'autre, la rencontre ayant duré plus d'un quart d'heure (Lanteri, 1982) (voir autres exemples de mouvements massifs dans les sections sur l'Egypte et Gibraltar, infra).

\subsubsection{Menaces}

Il n'est pas rare que des caouannes soient prises accidentellement dans des filets ou des aplets, daris la région d'Oran, en Algérie occidentale; comme elles ne servent pas à l'alimentation, elles sont généralement relâchées par les pêcheurs (Lanteri, 1982). De son côté, Laurent (1989a) a estimé, après avoir interrogé de nombreux pêcheurs dans plusieurs ports, que quelque 500 Caretta sont prises (ou reprises) chaque année en Algérie, la plupart du temps sur des aplets à espadon. Toutes les prises semblent accidentelles, mais peut-être de 10 à $30 \%$ ne sont pas relâchées; bien qu'il n'existe pratiquement pas de commerce des produits de la tortue, les pêcheurs peuvent garder les carapaces aux fins de décoration et employer parfois la chair pour l'alimentation (Laurent, 1989a). Les documents cités par ce dernier confirment que les tortues étaient très demandées au dix-neuvième siècle et, au moins localement, jusque vers la décennie 1940. Elles étaient alors capturées délibérément, et non seulement à l'occasion d'activités de pêche, comme c'est le cas aujourd'hui, et on les recherchait à des fins à la fois médicinales et alimentaires, et pour l'extraction d'huile (Laurent 1989a). 


\subsubsection{Mesures de protection}

Il n'y a aucune législation de protection en vigueur.

\subsection{CHYPRE}

\subsubsection{Plages de pontes et nombres : La caouanne (Caretta caretta)}

Au l'ouest de Chypre, la caouanne niche sur des plages avoisinant la péninsule d'Akamas, notamment dans la région de Lara, sur les rivages méridionaux de la péninsule, et autour de Polis, à l'est d'Akamas. Elle niche aussi sporadiquement et en très petit nombre sur plusieurs des plus tranquilles plages du sud de Chypre (Demetropoulos et Hadjichristophorou, 1986, 1987, communication personnelle du 9 juillet 1988). Les plages de la région de Lara comprennent, du sud au nord, Toxeftra (environ $1 \mathrm{~km}$ ), Ayiie Phanentes $(1,5 \mathrm{~km})$, et les plages sud, centre et nord de Lara (en tout, $3 \mathrm{~km}$ ). Du côté septentrional d'Akamas, on trouve plusieurs plages de sable à l'ouest et à l'est de Polis, qui totalisent peut-être $10 \mathrm{~km}$ de longueur. La seule étude pilote qu'on ait réalisée dans la région de Polis a révélé un nombre important de nids de caouanne, mais on ne sait à quel moment avait eu lieu la ponte (Demetropoulos et Hadjichristophorou, communication personnelle, 9 juillet 1988).

La caouanne niche largement sur les rivages septentrionaux de Chypre, fréquentant quelque 36 plages, mais ne se comptant en grand nombre sur aucune d'elles. Lors d'une étude effectuée entre le 18 juin et 14 juillet 1988, on enregistra 122 nids récents de caouanne (Groombridge et Whitmore, en préparation). Le taux de nidification moyen le plus élevé, sur les sites étudiés, était de 0,86 nid nouveau par nuit, sur une plage d'un demi-kilomètre de la péninsule de Karpas, alors que le taux moyen de nidification pour toutes les plages du nord de Chypre était de 0,19 nid nouveau par nuit. Les seules plages du nord de Chypre non étudiées se situaient autour de la baie de Morphou, à l'ouest; Ramsay (1970) et Demetropoulos (communication personnelle, 1988) confirment qu'il y eut là des nidifications dans le passé, et des résidants indiquent que des tortues nichent toujours. On ne connaît pas encore les espèces ni les nombres (bien que Ramsay ait signalé des nidifications de chélonée franche et des collectes occasionnelles d'oeufs).

La population de caouannes nichant au sud de Chypre a été estimée à quelque 300 individus ( Hadjichristophorou, comm. pers., 1986), bien qu'on n'ait pas encore publié de données corroborant ce chiffre. Cette estimation comprend les mâles adultes et se fonde sur la possibilité que les femelles nichent généralement une année sur deux (Demetropoulos et Hadjichristophorou, communication personnelle du 9 juillet 1988). Dans ces conditions, on estime que 75 caouannes pourraient nicher au sud de Chypre chaque saison. Une autre estimation veut qu'une cinquantaine de femelles nichent à Lara, et une centaine d'autres pondent sur les plages du secteur de Polis et à proximité de la base britannique (Hadjichristophorou, comm. pers., 2 octobre 1989). Des données préliminaires sur le nord de Chypre (Groombridge et Whitmore, inédit) suggèrent qu'il pourrait $y$ en avoir autant sur cette côte, de sorte qué le minimum saisonnier pour l'île pourrait se situer autour de 150 femelles reproductrices. 


\subsubsection{Plages de pontes et nombres : la chẻlonée franche (Chelonia mydas)}

Un petit nombre de chélonées nichent à l'ouest, surtout autour de la péninsule d'Akamas, et notamment dans la région de Lara. Les plages de la région de Lara comprennent, du sud au nord, Toxeftra (environ $1 \mathrm{~km}$ ), Ayiie Phanentes $(1,5 \mathrm{~km})$, et les plages de Lara sud, centre et nord ( $3 \mathrm{~km}$ en tout). On estime la population de chélonées à une centaine d'individus (Demetropoulos et Hadjichristophorou, 1987). Cette estimation comprend les mâles adultes et se fonde sur la possibilité que des femelles nichent généralement tous les deux ans (Demetropoulos et Hadjichristophorou, comm. pers. du 9 juillet 1988). Sur cette base, quelque 25 femelles pourraient utiliser les plages d'Akamas chaque saison. Au cours d'une période de 10 jours, début juillet 1988, alors que les habitants considéraient que l'année était bonne pour le nidification des chéelonées, le taux moyen de nidification était d'environ un nid par nuit pour toute la région de Lara (Demetropoulos, comm. pers. 10 juillet); ce taux est l'équivalent de quelque 0,2 nid par $\mathrm{km}$ et par nuit. Seize C. mydas ont été baguées alors qu'elles nichaient en 1989, ce qui constituait le plus grand nombre de tortues baguées en une même saison, et portait à 46 le total des femelles baguées à Chypre (Hadjichristophorou, comm. pers., 2 octobre 1989). On ne connaît pas d'autres lieux de ponte régulière de Chelonia mydas au sud de Chypre (Demetropoulos, comm. pers., 10 juillet).

On a enregistré un total de 96 nouveaux nids de chélonée franche sur 28 plages du nord de Chypre, au cours d'une étude préliminaire menée entre le 18 juin et le 14 juillet (Groombridge et Whitmore, inédit). La plupart des nids se trouvaient sur des plages voisines de la pointe de la péninsule de Karpas, soit dans le territoire ou juste hors du territoire proposé pour le Parc national terrestre et marin de Zafer Burnu (on recommande que les limites du parc soient reculées afin d'y inclure toutes les plages de ponte de la région). Le site le plus important consiste en une baie d'environ 3,75 $\mathrm{km}$ au nord-ouest du village de Dipkarpaz, qui comporte une plage d'environ 1,6 km de longueur, partiellement divisée par de petits éperons rocheux. Le taux de nidification, sur ce site, dépassait juste un nid par km et par nuit. A raison de trois couvées par femelle, il fallait un minimum de 32 femelles pour mettre en place le total observé de 96 nids; mais d'autres femelles ont sans doute nidifié avant et après la période de l'étude, et l'on estime que le total saisonnier des femelles, en 1988 , a dû être d'une cinquantaine. La seule plage du nord de Chypre non examinée se situait autour de la baie de Morphou, à l'ouest; Ramsay (1970) et Demetropoulos (comm. pers., 1988) confirment qu'il y a eu là des nidifications dans le passé, et des habitants indiquent que des tortues y nichent toujours. On ne connait pas encore espèces ni nombres, bien que Ramsay (1970) ait signalé des nidifications de chélonées et des collectes occasionelles d'oeufs.

\subsubsection{Tendances des populations}

On ne possède guère de renseignements sûrs, bien que Demetropoulos et Hadjichristophorou (1987) aient indiqué que la caouanne et la chélonée franche étaient plus abondantes dans le passé; ils se fondent sur des indications de pêcheurs, et sur le nom d'une localité (Khelones), au nord de Chypre, lesquels suggèrent que les lieux étaient réputés pour leur population de tortues. Des plages voisines de Larnaka et d'autres lieux touristiques accueillaient presque certainement plus de tortues reproductrices que le nombre 
négligeable qu'on y trouve aujourd'hui. Gruvel (1931) note que Chypre fournissait des tortues - apparemment surtout des Caretta - au commerce de la soupe de tortue établi à Londres; il est vraisemblable que cette exploitation ait eu un effet sur les populations reproductrices. Nous ne possédons pas de données historiques fiables sur les chiffres qui avaient cours dans le passé, ni par conséquent sur l'ampleur de la dégradation apparente.

\subsubsection{Menaces}

Tourisme et agriculture constituent à Chypre les principales sources de revenus, et la plupart des plages du sud de l'île souffrent déjà sérieusement du développement touristique et sont aujourd'hui perdues pour le nidification des tortues (Hadjichristophorou, comm. pers. du 2.9.1987); les plages relativement calmes qui subsistent, toutes situées dans la zone d'Akamas, sont à leur tour menacées par des projets de construction.

Les plages du nord de Chypre sont généralement assez peu perturbées jusqu'à présent, et certaines, situées surtout le long de la péninsule de Karpas, sont pratiquement intactes (sauf présence de débris marins). Mais le développement touristique s'étend vers l'ouest et vers l'est à partir du centre principal de Kyrenia, à mi-chemin de la côte septentrionale, et plusieurs parties de la baie de Famagouste sont exploitées pour les bains de mer. Il existe des projets de développement touristique le long d'importantes plages de ponte, et les perturbations semblent s'accroître en même temps que le tourisme.

La plupart des plages sont atteintes par la pollution, sous forme de boules de goudron et de déchets de plastique, probablement rejetés par la mer. Bien que les tortues reproductrices doivent parfois se frayer un chemin à travers ces détritus, on n'en connaît pas l'effet sur la réussite de la ponte. Bien entendu, une telle pollution ne rend pas les plages plus agréables à l'oeil. Celles de la région de Lara sont nettoyées manuellement, de temps à autre, et restent propres pendant de longues périodes (Demetropoulos, comm. pers.).

On capture un nombre important de tortues dans le nord de Chypre, apparemment par accident, à l'occasion de la pêche. La plupart, sinon toutes, sont prises dans des filets près du rivage, souvent près du lieu de ponte, et fréquemment sont noyées. On en estime le nombre entre 10 et 50 par an; et si une forte proportion d'entre elles sont des adultes, de telles prises incidentes risquent $d^{\prime}$ avoir un sérieux effet sur une population de tortues fort restreinte. Demetropoulos et Hadjichristophoru (1987) indiquent que des tortues sont parfois tuées sur la plage, pour leur écaille, et que d'autres sont noyées ou tuées lorsqu'elles se prennent dans les filets de pêche. Si l'on estime que les populations de tortues ont été atteintes, nous ne disposons d'aucune donnée sur le nombre des pertes.

\subsubsection{Mesures de protection}

\section{Zones protégées}

Les plages de Lara, sur la côte méridionale de la péninsule d'Akamas, qui comprennent la plupart des plages de ponte connues du sud de Chypre, sont actuellement louées par le ministère de la Pêche et gérées comme réserve naturelle : on espère ainsi réduire les perturbations d'origine humaine au cours des périodes de nidification et d'éclosion. 
On a promulgué, en 1989, d'importantes modifications aux lois sur la pêche (Hadjichristophorou, comm. pers., 2 octobre 1989). Il est désormais interdit : (1) de naviguer au large des plages de Lara, à moins de 20 mètres de profondeur; (2) de pêcher sans permis, par une méthode autre que la simple canne à pêche; (3) de pénétrer dans la zone de la plage au cours de la période qui va d'une heure avant le coucher du soleil jusqu'à son lever; (4) de conduire un véhicule sur la plage; (5) d'utiliser des tentes, parasols ou matelas de plage, etc., non conformes à la loi de protection de la laisse de mer. Aucune autre plage de ponte de tortues de la Méditerranée ne bénéficie de mesures de protection aussi complètes. La région de la péninsule d'Akamas, jusqu'ici à l'écart, est peu perturbée (elle comprend un important ensemble de faune et de flore), et semble maintenant garantie après sa désignation comme parc national; elle a fait l'objet, fin 1989, de discussions relatives à des mesures de planification, y compris de zonage.

Le projet de création, près de la pointe de la péninsule de Karpas, au nord-est de Chypre, du Parc national terresire et marin de Zafer Burnu englobe d'importantes plages de ponte de tortues, mais les limites doivent en être étendues pour comprendre également les importants lieux de nidification de Chelonia, au nord-ouest de Dipkarpaz. Trois importantes plages à tortues, à Dik Burun, Kirpasha et à l'est de Kyrenia, comptent parmi les six zones protégées du nord de Chypre, désignées fin 1989 (Koyuncuoglu, F., comm. pers., 13 décembre 1989).

\section{Eclosions}

Le ministère de la Pêche dirige une opération d'incubation artificielle dans le sud de Chypre, depuis 1978. C'est la première du genre en Méditerranée. On réenfouit les couvées recueillies dans des couvoirs de plage. On annonce actuellement un taux d'éclosion de $75 \%$, qui soutient assez bien la comparaison avec les 80 à $90 \%$ de réussite observés pour les nids non transplantés et non pillés (Demetropoulos et Hadjichristophorou, 1987). De nombreux jeunes ont fait l'objet d'une mise à l'eau protégée, principalement dans des bacs flottants, dans le port de Paphos. On détient actuellement une centaine de tortues. Des tortues d'un an relâchées et d'autres de deux ans auraient été vues dans la région de Paphos (Demetropoulos et Hadjichristophorou, 1987). On a entaillé en 1979 les rebords de la carapace d'une centaine de $\mathrm{C}$. mydas nouvellement écloses, avant de les relâcher. On a rapporté que deux femelles nichant en 1989 présentaient des marques provenant peut-être de ces entailles, et l'on a supposé qu'elles pouvaient avoir fait partie du lâcher de 1979

(Hadjichristophorou, comm. pers., 2 octobre 1989). Le fait impliquerait un taux de croissance extrêmement rapide.

L'objectif actuel du programme de transplantation, qui a permis de relâcher de 3.500 à 4.000 nouveau-nés par an, depuis quelques années, est de réduire la perte de couvées par prédation et inondation. Une étude a indiqué qu'environ $70 \%$ des nids étaient pillés par des renards; en outre, les nouveau-nés seraient mangés par des corbeaux et des crabes (Demetropoulos et Hadjichristophorou, 1987). Une bonne proportion des nids sont aménagés trop bas, sur la plage, et sont vulnérables aux grandes marées. Néanmoins, la plupart des conservateurs de tortues (Pritchard et coll., 1983) pensent qu'il faut réduire toutes techniques de manipulation au minimum. 


\section{Législation}

La loi de Chypre protège les tortues marines et leurs oeufs, par une règlementation édictée en vertu de 1'article 135 de la Loi sur la pêche. Les plages de Lara ont été récemment protégées grâce à des amendements à ce texte adoptés en 1989.

Tableau 1. Résumé de données choisies sur les plages de ponte de Caretta et de Chelonia à Chypre. Information sur le sud de Chypre de Demetropoulos et Hajichristophorou (comm. pers.); pour le nord de Chypre, de Groombridge et Whitmore (en préparation).

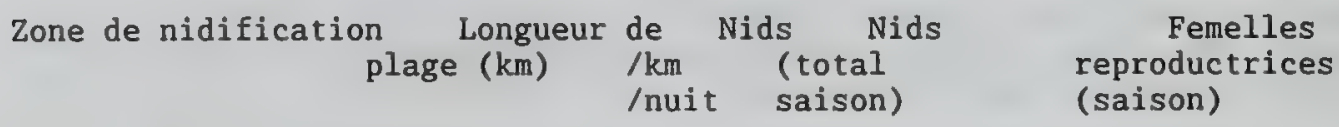

Chypre méridionale

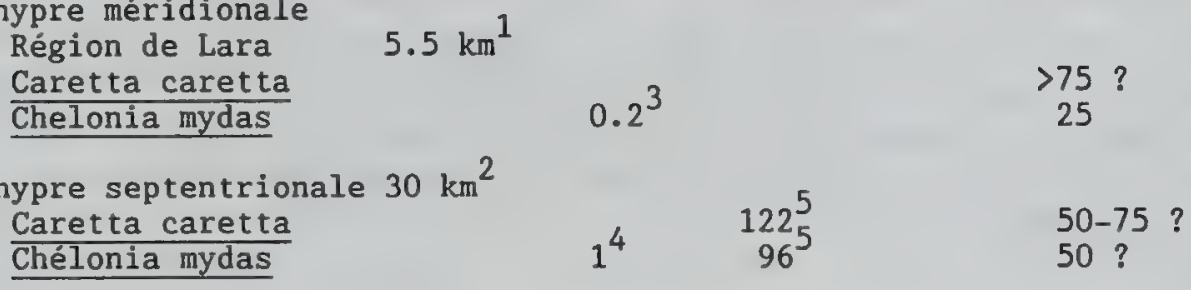

Notes : 1. La longueur de plage ne se réfère qu'à la zone de Lara (presque le seul lieu de ponte dans le sud, mise à part la région de Polis, non encore étudiée).

2. C'est à peu près la longueur totale des plages propres au nidification dans le nord (à l'exception de la baie de Morphou, non encore étudiée).

3. Pour la zone de Lara $(5,5 \mathrm{~km})$.

4. Pour la baie au nord-ouest de Dipkarpaz $(1,6 \mathrm{~km})$.

5. Estimations incomplètes fondées sur des données recueillies du 18 juin au 14 juillet 1988 (Groombridge et Whitmore, 1989).

\subsection{EGYPTE}

\subsubsection{Observations générales}

Nous possédons peu d'information récente. On rencontre tant Chelonia mydas que Caretta caretta dans les eaux méditerranéennes de l'Egypte (Flower, 1933), ainsi que dans les marchés de la côté, Caretta étant l'espèce la plus commune (Flower, 1933). On a observé des tortues marines au Lac Manzala (Flower, 1933) et au Lac Bardawil (N. Varty, comm. pers. 1989) respectivement à l'est et à l'ouest de Port Saïd. Loveridge et Williams (1957) parlent de signalements de Chelonia dans les environs de Port Saïd, et de Caretta près d'Alexandrie, Brullos, Damiette et Port Saïd (ces localités semblent être citées d'après Flower, 1933). 


\subsubsection{Plages de ponte et nombres}

Flower (1933) signale que des tortues marines nichent sur la côte du Sinaï; El Arish est la seule localité indiquée. Bien que Flower $n^{\prime}$ ait pas disposé d'indications quant aux espèces en cause, l'un de ses informateurs lui a décrit une tortue reproductrice qu'il avait vue comme "très grosse", ce qui suggère qu'il pourrait s'agir de Chelonia plutôt que de Caretta. Selon une carte publiée par Sella (1982), il existerait des nidifications isolés sur la côte du Sinaï, entre Port Saïd et la région de Gaza (on ne mentionne pas de témoignages, et la carte pourrait se fonder sur l'information de Flower). Plus récemment, on a confirmé des nidifications de tortues marines sur les bancs de sable séparant le Lac Bardawil de la mer, en nombre apparemment important (observations de Wahid Salama, de l'Agence égyptienne de l'environnement); l'on $\mathrm{n}^{\prime} \mathrm{a}$ pas de certitude quant à l'espèce, mais Chelonia a été observée dans le Lac.

\subsubsection{Les tortues en mer}

Au début du XXe siècle, Caretta était la tortue la plus commune dans les eaux méditerranéennes de l'Egypte (Flower, 1933).

Le 17 septembre 1947, un vapeur faisant route vers l'ouest a rencontré de nombreuses tortues à quelque 350 milles marins de Port Saïd (Egypte) ; on a aperçu des tortues sur une distance de quelque 60 milles, et toutes se dirigeaient vers $1^{\prime}$ est, direction Port Saïd, laissant entre elles un espace de quelque 200 mètres (Deraniyagala, 1951). Ces tortues mesuraient quelque $75 \mathrm{~cm}$ de longueur, étaient vert-gris, et semblaient être soit des chélonées franches, soit des Lépidochelys ; mais vu l'extrême rareté de cette dernière espèce en Méditerranée, et la couleur des animaux, il est très probable que les tortues en question étaient des chélonées. (On trouvera d'autres exemples de mouvements massifs de tortues de mer dans les sections sur l'Algérie et Gibraltar.)

\subsubsection{Menaces}

Il semblerait que les collectivités coptes vivant au bord de la Méditerranée utilisent de nombreuses tortues, probablement Caretta ou Chelonia (S. Goodman, comm. pers., 10 juin 1986). On garde les tortues vivantes sur les marchés pour leur trancher ensuite la gorge et boire leur sang, dont on croit qu'il favorise la fécondité. Flower (1933) signale qu'on peut souvent acheter des Chelonia vivantes à Port Saìd, mais qu'elles sont toujours aveugles à la suite de blessures causées par les pêcheurs; il semble qu'il en ait été de même pour les Caretta. Selon Flower, la chair des caouannes était très appréciée dans les hôtels et sur les paquebots, tant en Egypte que, semble-t-il, dans d'autres régions de la Méditerranée. On en capture encore beaucoup; par exemple, un marchand estimait qu'à lui seul il vendait de 20 à 40 grandes tortues chaque année (N. Varty, comm. pers., 1989). 


\subsection{FRANCE : CORSE}

\subsubsection{Observations générales}

On a signalé quatre espèces de tortues marines dans les eaux françaises de la Méditerranée (Fretey, 1987). Il semble que seules Caretta et Dermochelys se rencontrent en nombre important ; on n'a signalé que quelques fois des Chelonia, et une seule fois des Eretmochelys (qui auraient été prises près de Marseille - cité par Fretey, 1987).

Caretta est la seule espèce reproductrice, et l'on n'a signalé de nidifications qu'en Corse (Delaugerre, 1988 ; Fretey, 1987). Fretey cite également (1987) des hypothèses d'autres auteurs selon lesquelles Dermochelys pourrait nicher sur les nombreuses plages de sable du Golfe du Lion, où ont été signalés la plupart des individus de cette espèce rencontrés dans les eaux françaises de la Méditerranée.

\subsubsection{Plages de ponte et nombre : Caouannes Caretta caretta}

On rapporte que des caouannes auraient niché au cours de ce siècle, bien qu'on ne connaisse le détail ni des sites ni des nombres. Une lettre de 1970 de G. Testa (Musée océanographique de Monaco) à M. Dumont indiquait que des oeufs de Caretta avaient été trouvés sur les plages de sable fin de Corse orientale en 1923, 1928 et 1932. Cette information a été publiée par Dumont (1973, 1974), Bruno (1973), et est discutée par Delaugerre (1988). On a ainsi parlé de la plage Moriani, dans la région de Cervione, et d'Aleria, entre l'Etang de Diane et l'Etang de Sale.

Thibault et coll. (1984) citent le témoignage d'un habitant de Ghisonaccia selon lequel le nidification $n^{\prime}$ aurait pas été exceptionnel sur la plage du Domaine de Pinia au cours des années 60; mais Delaugerre (1988) a rejeté ce témoignage à la suite d'autres entretiens. Il a également signalé l'information donnée par un habitant de Solenzara - et apparemment confirmée par d'autres personnes interrogées - selon laquelle on ramassait parfois des oeufs dans les dunes voisines d'Aleria et dans l'anse de Favone, entre 1935 et 1940 .

Malgré l'absence de documents, Delaugerre (1988) conclut qu'un petit nombre seulement de Caretta nichaient sur les plages de Corse orientale, peut-être irrégulièrement, au tournant du siècle. Ces plages sont probablement des lieux secondaires de nidification de cette espèce, et les constructions et perturbations semblent avoir mis fin aux rares nidifications qui ont pu avoir lieu dans le passé. On n'en a signalé aucun au cours des dernières décennies.

\subsubsection{Tendances de la population}

Selon Delaugerre (1988), les témoignages dont on dispose suggèrent que le nombre de tortues diminue considérablement depuis vingt ou trente ans. Mais rien $n^{\prime}$ indique qu'il y a eu des nidifications en grand nombre dans le passé, et en l'absence de données historiques, il est impossible d'estimer l'ampleur d'une éventuelle réduction en nombre. Il semble que Caretta ait été commune en mer, dans le voisinage de Bonifacio, au cours du début des années 60 , alors que les pêcheurs en consommaient traditionnellement à la fête de saint Erasme (le 2 juin) (Delaugerre, 1988). Fretey (comm. pers. de janvier 1988) rapporte les dires de pêcheurs selon lesquels on voit aujourd'hui moins de tortues qu'il y a 20 ans. 


\subsubsection{Les tortues en mer}

On a observé des caouannes dans les eaux corses pendant la plus grande partie de l'année (sauf d'octobre à janvier). C'est en juin, juillet et août que l'on signale le plus d'individus, la fréquence diminuant brutalement à la fin août. Delaugerre (1988) suggère que la caouanne quitte la région à l'automne, pour se diriger vers les eaux plus tièdes du sud et de l'est. Les signalements intéressent presque toujours des individus isolés, très rarement des groupes de deux à cinq, et exceptionnellement des troupeaux plus nombreux. Ils se situent dans trois zones principales: le sud de la côte orientale, entre Aleria et Bonifacio, la région du Cap corse, et le Nord-est, y compris la péninsule de Scandola. Ces groupements pourraient, dans une certaine mesure, refléter la distribution des observateurs plutôt que celle des tortues. Cependant, des pêcheurs de la côte orientale indiquent que la caouanne se trouve très fréquemment dans deux zones bien définies (en particulier au sud-est de Cerbicale et entre Aleria et Favone). Il pourrait s'agir de zones d'alimentation préférées des caouannes (Delaugerre, 1988).

\subsubsection{Menaces}

Un nombre important mais incertain de tortues adultes et presque adultes (la plupart de 60 à $100 \mathrm{~cm}$ de longueur) sont capturées au voisinage de la Corse, surtout de la mi-juillet à la première partie d'août, lorsqu'elles s'approchent du rivage; elles se prennent alors dans des filets, et la plupart sont déjà mortes lorsqu'on les trouve (Fretey, comm. pers., janvier 1988). Sur 48 signalements dont parle Delaugerre (1988), il y en a 28 de Caretta prises dans des filets, des chaluts ou à des aplats; un pêcheur en a trouvé quatre, en cinq semaines, dans son filet à langoustes. Ces filets, qui reposent généralement sur le fond marin, entre 40 et $120 \mathrm{~m}$ de profondeur, constituent une importante cause de mortalité pour les caouannes des eaux corses (Delaugerre, 1988). Contrairement aux filets à poisson, les filets à langouste peuvent demeurer en place pendant plusieurs jours, et presque toutes les tortues qui s'y prennent sont noyées ( $1 a$ plupart de celles qui sont prises par d'autres moyens sont retrouvées vivantes). Comme la pêche à la langouste rapporte bien, une bonne partie de la flotte corse en a fait sa spécialité (Delaugerre, 1988).

\subsubsection{Divers}

Delaugerre (1988) signale quelques estimations de taille, généralement dans la catégorie des 70 à $80 \mathrm{~cm}$ de longueur totale, ce qui correspond approximativement à une longueur de carapace de 52 à 59 $\mathrm{cm}$. La moyenne de trois mesures directes de carapace était de $56,4 \mathrm{~cm}$ (il n'est pas précisé s'il s'agit de la longueur mesurée par une droite ou par la courbe de la carapace). La catégorie des 30 à $35 \mathrm{~kg}$ est la mieux représentée (le poids étant généralement estimé). La plupart des Caretta des eaux corses semblent ainsi d'une taille inférieure aux tortues qui se reproduisent en Méditerranée centrale et orientale (de 55 à $74,6 \mathrm{~cm}$ de longueur droite de carapace, à Dalyan (Geldiay et coll. 1982); longueur moyenne courbe de la carapace à Zante: 80,4 cm (Margaritoulis, 1982); longueur moyenne courbe en Baie de Kiparissia: 83,1 cm (Margaritoulis, 1988b). On a trouvé des oeufs dans une femelle de $76 \mathrm{~cm}$ de longueur totale.

Le contenu intestinal de deux tortues comprenait un crabe, pour l'une, des restes de céphalopode, un Pteroides, des sachets de plastique et enveloppes de bonbons, pour l'autre (Delaugerre, 1988). 


\subsection{GRECB}

\subsubsection{Observations générales}

La caouanne (Caretta caretta) est la seule espèce dont on sait ou dont on soupçonne qu'elle niche en Grèce. On ne connaît pas de nidification de Chelonia mydas, bien que quelques individus aient été vus dans les eaux grecques (Margaritoulis et coll. 1986).

\subsubsection{Plages de ponte et nombres : Caretta caretta}

On à signalé des nidifications de caouannes dans six régions, bien que l'information soit encore incomplète. Les zones de nidification connues comprennent: la partie méridionale de Zante (Zakynthos, en grec) et de Cephalonia (deux des îles ioniennes sur la côte occidentale de la Grèce); le Péloponnèse, et notamment la baie de Kiparissia, à l'ouest, celle de Lakonikos, au sud-est, et d'autres petites plages; les îles de Crête et de Rhodes (Margaritoulis, comm. pers., septembre 1987; Marinos, 1981).

Autant que nous sachions, Zante est de loin le plus important lieu de ponte des tortues en Grèce, et présente de loin la plus grande concentration de caouannes nichant en Méditerranée. Les chiffres sont modérés au Péloponnèse, faibles à Cephalonia, et apparemment faibles et quelque peu irréguliers sur d'autres sites.

\subsubsection{Zante}

Zante offre donc la plus forte concentration de caouannes reproductrices en Méditerranée; elle possède aussi l'un des rares sites de nidification de tortues de la Méditerranée à être exempts de renards (Vulpes vulpes). L'importance de la population reproductrice pourrait bien être liée au fait que le pillage de nids par les renards - qui fait beaucoup de victimes dans nombre d'autres sites connus (par exemple, ailleurs en Grèce, aussi en Turquie et à Chypre - est ici absent et que les prédations sont rares. On estime que $57 \%$ des couvées pondues en 1984 dans la Baie de Kiparissia, dans le Péloponnèse occidental, ont été pillées par des renards et des chiens mais, en 1987 , alors que près de la moitié des nids témoins contrôlés avaient été pillés, très peu d'entre eux avaient été totalement détruits (Margaritoulis, 1987a, 1987b).

Il existe sept plages de ponte le long de la baie de Laganas, dans le sud-est de l'île (voir détail dans Margaritoulis, 1982, 1987a). Elles comprennent, d'ouest en est: une petite plage sur la côte nord-est de l'ille de Marathonissi; Laganas "est" (2 km); Kalamaki (250 $\mathrm{m})$; Vrodonero $(150 \mathrm{~m})$; Sekania $(350 \mathrm{~m})$; Daphni $(250 \mathrm{~m})$; et Gerakas $(500 \mathrm{~m})$. La longueur totale de ces plages est d'environ $3,7 \mathrm{~km}$ (Margaritoulis, 1987a).

On pense que la plage de Laganas était le site principal mais la plupart des nidifications se font aujourd'hui à Sekania car le développement touristique a encore accru les nuisances et rendu la partie ouest de la plage de Laganas peu propice à la ponte (Margaritoulis, 1982). Ce transfert suggéré à Sekania n'est pas confirmé par les données disponibles, qui couvrent les saisons de 1983 à 1989. Lors des saisons de 1977 à 1979, on comptait une moyenne de 9,2 nids par nuit à Sekania (Margaritoulis, 1982), contre 9,4 nids pour la saison 1981 (Margaritoulis, comm. pers., 1982). Sekania accueille plus de $50 \%$ des nidifications de Zante, sur une bande 
Tableau 2. Résumé de données choisies sur les plages de ponte de Caretta caretta, en Grèce.

Clef des sources: $a=$ Sutherland (1984), b = Margaritoulis (1987a), $c=$ Margaritoulis (1987b), d = Société de protection des tortues marines (rapport 2 octobre 1989), e = Margaritoulis (1988b), $\mathrm{f}=$ Margaritoulis (comm. pers.), $\mathrm{g}=$ Sutherland (sans date).

$\begin{aligned} & \text { Zone de nidification } \\ & \text { e }\end{aligned}$
$\begin{gathered}\text { de plage } \\ (\mathrm{km})\end{gathered}$

Zante

$\begin{array}{lrrrrr}\text { moyenne } 1983-89 & 3.55 & 1337 & 417 & 1480 & \text { a-d } \\ \text { plage de Sekania } & 0.35 & \left(6268^{1}\right) & \left(2022^{1}\right) & 708 & \text { b-d }\end{array}$

Baie de Kiparissia (Péloponnèse occidental)

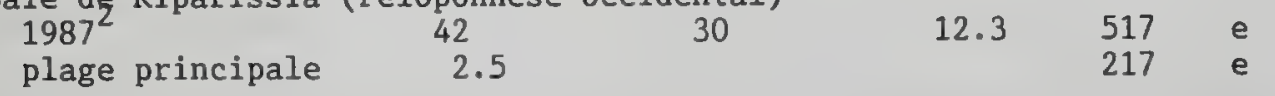

Golfe de Lakonikos (Péloponnèse méridional)
1988
20
$6.4 \quad 128 \quad d, f$

Cephalonia

Potamakia

340

$40 \quad 20$

$20 \quad 60 \quad \mathrm{~g}$

(moyenne 1985-87; nidification très rare dans le sud-ouest

Crète

Chiffres incertains, probablement aucun nidification dense

Rhodes

moyenne 1988-89

21 d

Notes: 1 Extrapglation. La plage de Sekania a une longueur d'un tier de kilomètre. 549 nids sur $44 \mathrm{~km}$ en 1988 (d).

Tableau 3. Importance relative des plages de ponte de la Baie de Laganas, à Zante, sur la base de données choisies de nidification (Moyennes des saisons 1984-1989). Les sources sont indiquées au tableau 4.

\begin{tabular}{|l|r|r|r|}
\hline Plages & $\begin{array}{c}\text { Longueur } \\
(\mathrm{km})\end{array}$ & $\begin{array}{r}\text { Nids en } \% \\
\text { des émergences }\end{array}$ & $\begin{array}{r}\text { Nids en } \% \\
\text { du total nids } \\
\text { pour Zante* }\end{array}$ \\
\hline Marathonissi & 0,20 & $26 \%$ & $7 \%$ \\
Laganas Est & 2,00 & $21 \%$ & $11 \%$ \\
Kalamaki & $0,25 \%$ & $8 \%$ \\
Sekania & 0,35 & $18 \%$ & $52 \%$ \\
Daphni & 0,25 & $29 \%$ & $6 \%$ \\
Gerakas & 0,50 & & 6 \\
\hline
\end{tabular}

* Moyenne des saisons 1984-1989 
Tableau 4. Résumé de données choisies sur les plages de ponte de Caretta caretta autour de la baie de Laganas, à Zante (Grèce).

\begin{tabular}{|c|c|c|c|c|c|c|c|c|c|c|c|c|c|c|}
\hline \multirow{2}{*}{\multicolumn{2}{|c|}{ Plage $^{\mathrm{E}}$}} & $983 a$ & \multicolumn{2}{|c|}{$1984 \mathrm{C}$} & \multicolumn{2}{|c|}{$1985 c$} & \multicolumn{2}{|c|}{$1986 d$} & \multicolumn{2}{|c|}{ 1987d } & \multicolumn{2}{|c|}{$1988 \mathrm{e}$} & \multicolumn{2}{|c|}{1989 e } \\
\hline & & $\mathrm{N}$ & E & $\mathrm{N}$ & $E$ & $N$ & $E$ & $\mathrm{~N}$ & $E$ & $\mathrm{~N}$ & $E$ & $\mathrm{~N}$ & E & $\mathbf{N}$ \\
\hline M & 355 & 151 & 284 & 67 & 318 & 77 & 321 & 88 & 360 & 105 & 376 & 89 & 382 & 123 \\
\hline L & $\begin{array}{r}514 \\
1047\end{array}$ & $\begin{array}{l}252 \\
205 b\end{array}$ & 482 & 124 & 542 & 137 & 961 & 190 & 778 & 117 & 534 & 127 & 779 & 168 \\
\hline $\mathrm{K}$ & 453 & 205 & 301 & 92 & 392 & 104 & 349 & 94 & 577 & 93 & 560 & 103 & 705 & 188 \\
\hline$S$ & $\begin{array}{l}2634 \\
2590\end{array}$ & $\begin{array}{l}1360 \\
1142 b\end{array}$ & 1761 & 595 & 1195 & 333 & 2506 & 1046 & 2341 & 611 & 2838 & 823 & 2083 & 840 \\
\hline D & 847 & 322 & 627 & 123 & 587 & 133 & 1274 & 294 & 1458 & 138 & - & $153 *$ & 1084 & 207 \\
\hline G & 344 & 147 & 219 & 60 & 178 & 73 & 497 & 110 & 262 & 46 & 354 & 113 & 309 & 118 \\
\hline & $\begin{array}{c}5411 \\
c .\end{array}$ & $\frac{2460}{2000 b}$ & 3674 & 1061 & 3212 & 857 & 5908 & 1822 & 5776 & 1110 & 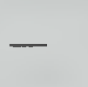 & $1408 *$ & 5342 & 1644 \\
\hline
\end{tabular}

Indications des femelles reproductrices possibles nombre/année (voir Note 3)

$\begin{array}{lllllll}820 \mathrm{a} & 354 & 286 & 607 & 370 & 470 & 548 \\ 667 \mathrm{~b} & & & & & \end{array}$

Clef du tableau 4 - lère colonne: $M=$ Marathonissi $(0,2 \mathrm{~km})$;

$\mathrm{L}=$ Laganas Est $(2 \mathrm{~km}) ; \mathrm{K}=\operatorname{Kalamaki}(0,25 \mathrm{~km}) ; \mathrm{S}=\operatorname{Sekania}(0,35 \mathrm{~km})$; $D=$ Daphni $(0,25 \mathrm{~km}) ; G=$ Gerakas $(0,5 \mathrm{~km})$. Longueur de plage totale : $3,55 \mathrm{~km} . \mathrm{E}=$ total des émergences, $\mathrm{N}=$ total des nids. * La plage de Daphni n'a pas été régulièrement examinée en 1988; le nombre de nids a été estimé sur la base de repérages occasionnels (source e).

Clef des symboles indiquant les sources: a = Sutherland (1984), $b=$ Margaritoulis (comm. pers.), c = Margaritoulis (1987a), d = Margaritoulis (in litt., données issues du rapport de 1988), e $=$ rapport de la Société de protection des tortues, octobre 1989.

Notes relatives au tableau 4: (1) Les données relatives au nidification pour la saison 1983 ont été recueillies par deux équipes indépendantes - Sutherland (1984) et une équipe patronnée par le Ministère grec de l'Environnement; les informations provenant des deux sources figurent au tableau 3, sous les symboles respectifs "a" et "b". Bien que le rapport de Sutherland reconnaisse certaines limites à la portée de ces données (Laganas et Skopos = les plages de Vrodonero n'ont été observées que d'un bateau, à la jumelle, et Sekania a seulement fait l'objet d'une étude partielle), ces chiffres couvrent toutes les plages et figurent donc dans la partie principale du tableau. L'équipe Margaritoulis a procédé à une étude plus approfondie mais ne portant que sur Laganas et Sekania; ses informations sur les plages en question sont probablement les plus exactes des deux groupes de données disponibles. (2) La plage de Vrodonero n'a pas été prise en compte dans les études ultérieures à 1983. (3) Ce n'est là qu'une indication du nombre total de femelles reproductrices fondée sur l'hypothèse que chaque femelle adulte pond en moyenne sur la plage trois couvées par saison. Ces chiffres sont indiqués à des fins comparatives uniquement. Les deux chiffres de 1983 proviennent des deux estimations générales disponibles concernant les tentatives de nidification à Zante. 
représentant $10 \%$ de la longueur totale des plages de la Baie de Laganas. La plage a également la plus forte proportion d'émergences suivies de nidifications réussis, ce qui indique que les conditions sont plus favorables que sur les autres rivages. La plupart des plages de Zante accusent une proportion relativement faible de nidifications réussis. Par exemple, sur celle de Potamakia (Cephalonia), de 45 à $50 \%$ des émergences aboutissent à des nidifications, contre une moyenne de seulement $23 \%$ à Zante, à l'exclusion de Sekania, qui enregistre $32 \%$.

On a compté un total de 2.460 nids à Zante en 1983 (Sutherland, 1984), soit une moyenne de quelque 665 nids au $\mathrm{km}$, pour la saison (dont $55 \%$ à Sekania, qui ne représente que $10 \%$ des plages disponibles). Si, à des fins comparatives, on suppose une moyenne de 2,5 ou 3 pontes par femelle et par saison, ces données révèlent un contingent nicheur annuel de 820 ou 980 femelles. Une autre estimation relative au nidification en 1983 (Margaritoulis, comm. pers., 1988) suggère un total d'environ 2000 nids, ce qui correspond, compte tenu des suppositions précédentes, à $666-800$ femelles. On trouvera au tableau 4 d'autres estimations pour les saisons de 1984 à 1989 .

\subsubsection{Cephalonia}

On a signalé des nidifications sur trois plages (Skala, Munda et Potamakia), près du village de Skala, au sud-est de Cephalonia, et, très clairsemés, sur deux plages près de Lixouri, au sud-ouest (Sutherland, sans date; C. Whitmore, comm. pers.). Il y a, semble-t-il, quelques nidifications en plusieurs lieux (Panou et Moschonas, 1990). Environ $80 \%$ des nidifications signalés se font sur les trois kilomètres de la plage de Potamakia (Sutherland, sans date). Le tableau 5 présente des données choisies pour les saisons 1984-1989.

Tableau 5. Données choisies de nidifications sur la plage de Potamakia, à Cephalonia. - Sutherland (sans date) et anonyme (1990). On a contrôlé les plages pendant toute la saison de nidification d'environ 74 jours (sauf en 1984, alors que les observations ont été faites pour 50 jours; les valeurs extrapolées pour une saison de 74 jours sont données entre parenthèses).

\begin{tabular}{|l|c|c|c|c|}
\hline Saison & Emergences & Nids & $\begin{array}{r}\text { Nids en } \% \\
\text { des } \\
\text { émergences }\end{array}$ & $\begin{array}{c}\text { Nombre de } \\
\text { femelles } \\
\text { aperçues }\end{array}$ \\
\hline & & & & \\
1984 & $74(114)$ & $36(53)$ & $48 \%$ & 9 \\
1985 & 95 & 44 & $46 \%$ & 26 \\
1986 & 151 & 84 & $55 \%$ & 26 \\
1987 & 119 & 51 & $43 \%$ & 13 \\
1988 & 56 & 28 & $50 \%$ & $?$ \\
\hline
\end{tabular}

Une bonne partie des nids de Potamakia se trouve dans le secteur oriental de la plage; la plupart des débris marins s'échouent au même endroit, ce qui suggère que les femelles pourraient utiliser les courants locaux lorsqu'elles s'approchent du rivage (Whitmore, C., comm. pers.). Potamakia est relativement calme à présent (Sutherland, sans date). C'est aussi sur cette plage que l'on compte le plus 
d'éclosions. Au cours de la saison 1985, les nidifications ont été les plus nombreux dans la deuxième semaine de juin, et ils ont ensuite diminué régulièrement jusqu'à fín août. Le nombre annuel moyen de nids est d'une cinquantaine, ce qui correspond à une densité de 17 nids par km et par saison, et à un taux de nidification de quelque 0,7 nid/nuit à Potamakia. L'une des 26 femelles adultes baguées en 1985 avait déjà été baguée lors d'un nidification à Zante (Sutherland, sans date; Whitmore, C., comm. pers.). Le nombre moyen de couvées de neuf femelles baguées lors de nidifications et ayant reniché était de 2,8 .

Le contingent de reproductrices de Cephalonia est assez restreint. Le nombre de nids comptés, divisé par le nombre moyen de couvées par femelle dénote par exemple, en 1985, la présence de 18 ou 19 femelles; on a néanmoins bagué 26 femelles mûres, ce qui suggère qu'une majorité des tortues présentes au large des plages de Skala pourraient n'avoir niché qu'une fois ou deux dans les abords immédiats.

\subsubsection{Péloponnèse}

On a observé des empreintes de tortues dans plusieurs localités des côtes occidentales et méridionales de la péninsule (Margaritoulis, comm. pers., 4.6.88). La plupart des observations ont été faites à la Baie de Kiparissia, dans le Péloponnèse occidental, et dans le golfe de Laconie, au sud de la péninsule. Il semble que la plupart des nidifications se font sur la grande plage de la baie de Kiparissia, où l'on a signalé 1.272 émergences et $517 \mathrm{nids}$ en 1987, sur une distance de 42 kilomètres entre le fleuve Alfios et Kiparissia (Margaritoulis, 1987b). La densité générale de nidification, en 1987, était de 12,3 par km; mais les nids étaient très inégalement distribués. La plupart des nidifications, jusqu'à un

maximum de 86,8 nids pour un seul secteur, ont eu lieu au Sud, entre les bouches des fleuves Neda et Arcadicos. Il y avait beaucoup moins de nids dans le Nord, sur les secteurs les moins perturbés, qui paraissaient superficiellement offrir des conditions idéales pour la nidification; on ignore les raisons de cette disparité (Margaritoulis, 1987b). On a dénombré, en 1988, un total de 549 nids sur $44 \mathrm{~km}$ (voir de protection des tortues, rapport du 2 octobre 1989). A la différence de Zante, la Baie de Kiparissia est le théatre d'une très forte prédation des nids; $48 \%$ de ceux-ci ont été pillés en 1987, principalement par des renards. $\mathrm{L}^{\prime}$ activité touristique et d'autres formes d'aménagements contraires aux tortues ne sont pas aujourd'hui prédominants. On contrôle quelque $20 \mathrm{~km}$ de plages dans le golfe de Laconie, où $l^{\prime}$ on estime que de 60 à 120 couvées sont déposées chaque saison (Margaritoulis, comm. pers.). En 1988, on a relevé 128 nids sur $20 \mathrm{~km}$ (Société de protection des tortues, rapport du 2 octobre 1989). Il y a relativement peu d'aménagements dans la région, mais beaucoup de pêche et un taux élevé de prédation des nids (Margaritoulis, comm. pers.). Des enquêtes préliminaires ont révélé des empreintes de tortues et des nids dans le lagon de Strofilia-Kotychi et sur la plage de Romanos (Margaritoulis, comm. pers.).

\subsubsection{Crète}

Les études couvrant (bien que de manière non systématique) la plus grande partie de la côte sud où des empreintes n'ont été relevées qu'occasionnellement, et certaines parties de la côte nord oủ, au cours de la saison 1985, un total de cinq nids a été recensé sur une plage de quelque $20 \mathrm{~km}$, à l'ouest de Rethimnon (Margaritoulis, comm. pers., 4.6.88). Le nidification semble très rare en Crète, et l'on ne connaît ni ne soupçonne l'existence d'ensembles de nids importants. Nous $n^{\prime}$ avons aucune information sur la densité passée de la nidification, ni sur les tendances de la population. 


\subsubsection{Rhodes}

On a signalé quelques traces d'émergences, principalement sur les plages les plus isolées de la partie sud de l'île; le nombre de reproductrices ne semble pas élevé. Sur quelque $35 \mathrm{~km}$ de plages, au sud de l'île, on a dénombré 9 et 33 nids respectivement en 1988 et 1989 (Société de protection des tortues, rapport du 2 octobre 1989).

\subsubsection{Tendances de la population} Cephalonia.

L'information disponible ne se rapporte qu'à Zante et à

\section{Zante}

On ne connaît pas avec certitude les tendances à long terme du nombre de reproductrices; on ne possède pas de données quantitatives quant à la taille des anciens contingents de reproductrices mais on considère la population actuelle comme fortement appauvie (Margaritoulis, 1980). Selon Margaritoulis (1980), des habitants âgés affirment que de grands troupeaux de tortues apparaissaient parfois dans les eaux maigres, pour ensuite venir à terre et nidifier.

Margaritoulis (comm. pers., décembre 1988) n'est plus aussi sûr qu'il $y$ ait une diminution de la population.

Les chiffres ont varié selon les saisons pendant lesquelles les études ont été faites et, d'après Margaritoulis (comm. pers., 2.9.67), on ne peut discerner aucune tendance nette au cours de cette période. Toutefois, le nombre total de nids enregistrés pour chacune des quatre saisons de 1984 à 1989 est inférieur au total pour 1983, première année pour laquelle des données utiles sont disponibles et le total de 1987 représente environ 50\% de celui de 1983. Par ailleurs, on a enregistré entre 1984 et 1989 un nombre d'émergences semblable ou légèrement supérieur au chiffre de 1983 et il se peut que la population de femelles reproductrices soit restée stable mais elle a été plus souvent empêchée de nicher. On ne dispose aujourd'hui d'aucune donnée permettant une comparaison des éclosions réussies sur

différentes plages. Dans l'ensemble, les témoignages de déclin sérieux des populations en raison du développement touristique intense du milieu des années 70 , sont au mieux discutables, et les preuves d'un déclin de la population de Zante au cours des années 80 ne sont pas concluantes. Il n'y a cependant pas de doute que cette population, qui constitue la plus forte colonie de tortues reproductrices connue en Méditerranée, est gravement menacée par le développement touristique; vu les sérieuses perturbations dont souffrent aujourd'hui le nidification et les habitats internidifications, il n'est pas douteux que la population ira diminuant, si l'on ne prend des mesures correctives de toute urgence.

\section{Cephalonia}

Les données disponibles sur la plage de Potamakia (Sutherland, sans date; Anonyme d, 1990) indiquent une variation marquée du nombre de nids selon les saisons, mais aucune tendance nette au cours de la période 1984-1987. 


\subsubsection{Menaces}

\section{Zante}

La population de caouannes de Zante est menacée par divers facteurs associés au développement touristique, et notamment: perturbation directe des tortues et des nids par l'activité humaine; perturbation et désorientation des femelles et des nouveau-nés par l'éclairage artificiel; construction et plantation d'arbres sur certaines plages; évolutions de bateaux rapides dans la baie de Laganas. Le tourisme S'est beaucoup développé depuis l'ouverture d'un aéroport international sur l'île. Le potentiel économique du développement touristique est assez important pour que les propriétaires locaux de terrains et la collectivité en général ne voient pas d'un bon oeil les exigences d'une bonne gestion de la population de caouannes reproductrices. Une directive de 1980 déclarant

les principales plages zones protégées, des restrictions provisoires à la construction en 1982 et un décret présidentiel de 1984 imposant des limitations à la construction et à l'aménagement du territoire sont restés lettre morte.

Le secteur ouest de la plage de Laganas a été tout à fait envahi, depuis 1979, par les aménagements résidentiels et touristiques, et n'est plus fréquenté par les tortues; le secteur est était très utilisé pour le nidification, mais les perturbations y sont aujourd'hui importantes, en particulier aux environs de l'Hôtel de la Plage de Kalamaki, situé à l'extrêmité orientale. Il y a encore des nidifications sur la plage de Kalamaki, mais celle-ci est fortement perturbée, surtout autour de l'Hôtel Crystal Beach. Sekania demeure relativement calme, et le nidification y est si concentré - probablement en raison des perturbations qui existent ailleurs - que les tortues reproductrices, dit-on, creusent souvent à l'emplacement d'autres nids et endommagent les couvées. Cependant, Margaritoulis (comm. pers. 1988) indique que sur les $1.046 \mathrm{nids}$ relevés en 1986, une douzaine seulement ont été détruits de cette manière. La plage de Daphni, dont l'importance la place tout de suite après Sekania, court maintenant des risques en raison des constructions illégales réalisées en 1986. Le nidification continue à Gerakas et sur l'ile de Marathonissi, deux endroits perturbés par l'activité touristique, surtout évidente pendant la journée.

Selon Venizelos (1987), la plupart des plages étaient couverts de parasols et de chaises longues; sur le rivage s'alignaient les pédalos et autres embarcations; on avait retiré du sable à plusieurs endroits à des fins de construction; certaines plages étaient surchargées par les véhicules, les cavaliers et en général une activité touristique intense; la baie elle-même était le théâtre de pêche à la dynamite, de chalutage, et de courses de bateaux rapides. En outre, les aménagements du front de mer - dont les digues, cafés, plantations de tamaris (pour leur ombre) - avaient proliféré et, bien que souvent déclarés illégaux par l'administration locale, échappaient à toute action en raison de l'opposition vigoureuse des propriétaires locaux. Les plages de l'ouest en particulier étaient profondément perturbées, la nuit venue, par la pollution sonore et lumineuse des "discos" et par l'activité touristique générale; il n'est pas douteux que tout cela dérange sérieusement les femelles reproductrices et désoriente les nouveau-nés. Selon d'autres sources (Margaritoulis, comm. pers., décembre 1988), la menace représentée par nombre des aménagements précités est quelque peu exagérée: la quantité de sable prélevée est minime et limitée aux dunes derrière la plage de ponte; une réglementation sur la pêche interdit le chalutage dans les baies fermées (comme celle de Laganas) et le peu de pêche pratiquée l'est en hiver (hors saison de ponte). 
La gravité de la situation d'ensemble sur les plages de ponte de Zante a été confirmée, d'autre part, au cours d'une évaluation effectuée par la Direction de l'Environnement et des Pouvoirs Locaux du Conseil de l'Europe (Anon. a, 1987) et par un consultant de la Societas Europaea Herpetologica (Anon. b, 1987). Une appréciation de suivi, effectuée en septembre 1989 (Anon. c, 1989), a conclu qu'à plusieurs égards la situation avait empiré depuis la première appréciation, et qu'on avait enregistré peu de progrès réels dans la mise en oeuvre de la recommandation $n^{\circ} 9$ adoptée par le comité permanent en 1987.

\section{Prises accidentelles}

Panou et Moschonas (1990) rapportent que 11 Caretta, présentant une moyenne de $45 \mathrm{~cm}$ de longueur (entre 20 et $65 \mathrm{~cm}$ ), ont été prises à l'occasion de 54 sorties entre mars et août 1989, d'un bateau de pêche à l'espadon par aplets ayant son port d'attache à Céphalonia. Toutes ont été relâchées vivantes, généralement par coupure de l'aplet à la remontée. Un deuxième et un troisième bateau ont indiqué avoir pris respectivement trois et quinze tortues au cours de la même saison. La plupart des captures se produisent en juillet et août, probablement parce que les tortues se rassemblent au large des plages de ponte. Des membres égyptiens de l'équipage mangent occasionnellement de la tortue. Des bateaux japonais et italiens utilisant des filets trainants (respectivement pour le thon et l'espadon) sont également soupçonnés de constituer une menace pour les tortues dans la région.

\subsubsection{Les tortues en mer}

On a bagué un total de 1.525 Caretta en Grèce, au cours des saisons de nidification de 1982 à 1987, pour la plupart à Zante et le reste sur les rivages du Péloponnèse; jusqu'à mars 1988, 34 tortues baguées $(2,2 \%)$ avaient été recapturées (Margaritoulis, 1988a). La plupart des recaptures ont été signalées par des pêcheurs, et environ la moitié des tortues reprises auraient été libérées. On a retrouvé des bagues de très nombreuses provenances, allant de la Sardaigne ¿̀ l'ouest, à la Turquie occidentale à l'est, à la Tunisie (golfe de Gabès) et à la Libye, au sud, et, au nord, à la partie septentrionale de la mer Egée et de l'Adriatique. Quatorze ( $41 \%$ ) des 34 retours venaient du golfe de Gabès, surtout pendant l'hiver, les autres sites ayant signalé de un à trois retours (Margaritoulis, 1988a). On ne sait dans quelle mesure ces résultats reflètent la distribution des tortues baguées ou l'importance des captures en Tunisie. Tels quels, ces retours de bagues indiqueraient que le golfe de Gabès est un secteur d'hivernage et d'alimentation majeur pour les caouannes; mais la pêche, dans ces secteurs, est plusieurs fois plus active que dans d'autres lieux de la Méditerranée centrale, et l'on doit évidemment $s^{\prime}$ attendre à des retours de bagues également élevés. Il serait cependant peu vraisemblable qu'une pêche spécialisée à la tortue se serait développée dans une zone pauvre en tortues, de sorte qu'il est raisonnable de supposer que le golfe de Gabès est effectivement un important lieu d'hivernage pour la caouanne. 


\subsubsection{Mesures de protection}

\section{Zanthe}

Une récente étude (1984-85) du ministère de l'Environnement, de l'Aménagement du territoire et des Travaux publics (Margaritoulis, 1987a) proposait que l'actuel centre du nidification - comprenant les plages de Sekania, Daphni et Gerakas, avec la zone marine adjacente jusqu'à 500 ou 600 mètres du rivage - soit acheté et géré comme sanctuaire à tortues, en conjonction avec un centre de recherche et d'information qui se situerait à Daphni. On prendrait en même temps une série de mesures intéressant les plages de Laganas et de Kalamaki, afin de limiter et de contrôler les effets du tourisme massif. Ces mesures comprendraient l'interdiction d'accès après le coucher du soleil, la prohibition de tout véhicule sur les plages, la réduction des niveaux de bruit et d'éclairage, le contrôle de l'activité des bateaux, la patrouille de la plage, et d'autres. L'ensemble de ces mesures est le strict minimum nécessaire pour réduire l'actuelle menace contre les populations de tortues.

Répondant à une inquiétude croissante quant aux pressions qui $s^{\prime}$ exercent contre les populations reproductrices de Zante, le ministre de l'Environnement et des Travaux publics a pris, le 29 janvier 1987 (Journal officiel 37D/29-1-1987), un décret ministériel qui définit des zones de restriction de la construction, notamment de logements, des zones où les plages de ponte seront protégées, et qui comprend aussi d'autres mesures de protection des tortues. Aucun développement touristique n'est autorisé dans les zones protégées, et le logement est soumis à de stricts règlements de construction. Lorsque le développement touristique est permis, une règlementation détermine la dimension, la hauteur et la capacité des bâtiments. Aucun éclairage n'est toléré sur les aires de ponte ou, en mer, près de celles-ci. Les plages de ponte sont fermées la nuit, et la circulation de véhicules y est interdite. On autorise les parasols, chaises longues et pédalos dans des secteurs déterminés. L'administration locale a touché 55 millions de drachmes (environ 420.000 dollars) pour appliquer le décret.

Un décret commun ( $n^{\circ} 18670 / 777 / 29.2 .1988$ ) de trois ministères, début 1988 , a créé un secteur marin protégé dans la partie orientale de la baie de Laganas (Journal officiel 137B/10-3-88), comprenant une zone centrale de 2.000 ha où toute navigation et toute pêche, par tous moyens, sont interdites, et une zone tampon de 13.000 ha où la vitesse est limitée à six noeuds.

La patrouille des plages de ponte, selon les dispositions du décret ministériel, par un personnel local et par des volontaires, semble avoir beaucoup réduit les perturbations à Sekania, Daphni et Marathonisi, au cours de la saison 1987 (Venizelos, 1987). Certaines couvées déposées sur la plage de Kalamaki et sur le secteur oriental de la plage de Laganas et jugées en péril ont été transplantées sur un petit terrain d'incubation.

Néanmoins, comme l'ont souligné Venizelos (1988, 1989) et d'autres (Anon. c), la situation de la protection des tortues à Zante s'est peu améliorée depuis 1987 et suscite toujours de graves inquiétudes. Le développement touristique massif prévu pour le secteur ouest de la baie, et l'existence d'une zone de constructions de densité faible et moyenne couvrant à peu près tout le reste de la baie augmenteront la pression sur les lieux de nidification et d'alimentation des tortues. La construction illégale et d'autres aménagements, y compris sur la plage de Daphni (deuxième en importance, après Sekania) 
se sont poursuivis à peu près sans entrave depuis que la décision ministérielle est entrée en vigueur. On n'a pas encore mis en oeuvre la disposition prévoyant l'achat de terrains pour éviter la perturbation des tortues reproductrices; les fonds reçus de la CEE et destinés à la protection de Caretta sont restés inemployés. La situation a été aggravée par des intérêts locaux conflictuels et de fréquents changements du personnel administratif clef, tant sur le plan local que sur celui du gouvernement central.

Le Comité permanent de la Convention relative à la conservation de la vie sauvage et du milieu naturel de l'Europe (Convention de Berne), agissant sur l'avis d'un groupe d'experts qui avait pour mandat de fournir ses conseils sur les questions intéressant les tortues marines, a, à sa réunion de décembre 1987 , approuvé une recommandation ( $n^{\circ} 9$ ) pour la protection de Caretta dans la baie de Laganas. Il y était recommandé au gouvernement grec une série de mesures générales et particulières à prendre pour la survie de l'importante population de Caretta de sa juridiction. Depuis fin 1989, la plupart de ces recommandations n'or.t pas été appliquées.

La Grèce a ratifié la Convention de Berne, mais, pour ce qui est du nidification des caouannes dans la baie de Laganas, elle ne remplit pas les obligations qu'elle a ainsi assumées (Anon. c, 1989).

\section{Information générale}

Un programme à long terme de baguage et de contrôle des plages a été lancé en 1982 par le ministère de la Coordination, avec l'aide financière de l'Alliance pour la nature, et a été poursuivi par le ministère de l'Environnement, en coopération avec les universités grecques et la Société hellénique de protection des tortues marines, grâce à un cofinancement de la CEE. Depuis 1988 , le baguage et le contrôle ont été entrepris par la société précitée, avec l'aide de la CEE, du ministère de l'Environnement et de l'Alliance pour la nature. Le projet couvre Zante, des parties du Péloponnèse et Rhodes. La Société a également mis en oeuvre, depuis 1987, un programme de sensibilisation du public de Zante, en coopération avec la préfecture, le ministère de l'Environnement et l'Alliance pour la nature (projet 3825, Grèce), ainsi qu'un programme d'éducation pour écoliers.

\section{Législation}

Trois espèces de tortues marines : Caretta caretta, Chelonia mydas et Dermochelys coriacea, sont inscrites sur la liste des espèces protégées en vertu du décret présidentiel $n^{\circ} 67 / 1980$ pris par le ministère de l'Agriculture. Le décret $n^{\circ} 617 / 1981$ interdit la capture des tortues marines et le ramassage ou la destruction des oeufs ou des nouveau-nés. La loi $1335 / 1983$ ratifiait la Convention relative à la conservation de la vie sauvage et du milieu naturel de l'Europe (Convention de Berne). Voir 3.7.6. ci-dessus pour législation sur l'aménagement à Zante.

\subsubsection{Divers}

La longueur moyenne ligne droite de la carapace des tortues mesurée au sud-est de Cephalonia (C. Whitmore, comm. pers.) est de $76,6 \mathrm{~cm}$ $(n=25)$; poids moyen : $68 \mathrm{~kg}(n=12)$, gamme de 52 à $84 \mathrm{~kg}$; ponte moyenne : 100 œufs; incubation moyenne : 59,6 jours; réussite d'ensemble de l'incubation : $47 \%$. 
Les femelles nichant dans la baie de Kiparissia en 1987 avaient une longueur moyenne ligne droite de carapace de $78,58 \mathrm{~cm}$ (gamme de 69 à $91 \mathrm{~cm}$ ) ; longueur moyenne de carapace ligne courbe : $83,14 \mathrm{~cm}$ (de 73 à 98) (Margaritoulis, 1987a). La longueur moyenne de carapace ligne courbe des femelles nichant à Zante en 1978-1979 était de $80,4 \mathrm{~cm}$ (gamme d'environ 70 à $94 \mathrm{~cm}$ ) (Margaritoulis, 1982).

\subsection{ISRAËL}

\subsubsection{Observations générales}

On a dit qu'à la fin du 19 e siècle, les Caretta n'étaient pas rares au large des côtes de la grande région palestinienne (Tristram, 1888, cité par Flower, 1933); selon Hornell (1935) on voyait de très nombreuses Chelonia mydas, chaque saison, au large de la côté nord. On ne connaît pas avec certitude la fréquence du nidification dans le passé, mais on sait qu'aujourd'hui il y a très peu de tortues marines sur la côte israélienne de la Méditerranée. On a signalé 10 nids seulement en 1984, 14 en 1985 et 17 entre Haïfa et Césarée en 1986 (Kuller, in litt., 23 novembre 1986). Dans l'ensemble, il semble que le nombre annuel de nids $n^{\prime}$ ait pas dépassé cinq à vingt, pour la plupart de Caretta, avec seulement quelques Chelonia (Mendelssohn, in litt., ler août 1988; Kuller, in litt.; Ashkenazi et Sofer, 1988).

\subsubsection{Plages de ponte et nombres: caouannes (Caretta caretta)}

Dans les années 50, Sella (1982) a compté quelque 15 nids par kilomètre, à chaque saison, sur les 5 kilomètres de côte entre Rosh-Hanigra et Nahariya, au nord d'Acre et près de la frontière libanaise ; une densité semblable a été constatée sur les 8 kilomètres de plage d'Atlit, un peu au sud de Haïfa. On n'a trouvé que des nids occasionnels sur les $400 \mathrm{~km}$ restants de côte d'Israël et du nord du Sinaï. Ces estimations donnent à penser que, pour les années 50 , il y avait peut-être, annuellement, quelque 200 nids de Caretta. Nous n'avons pas d'information sur le nidification au cours des décennies précédentes.

En 1964, la densité de nidification s'était réduite à quelque trois nids par kilomètre à Rosh-Hanigra, et à un seul à Atlit ; il y avait un total de 26 nids sur les $13 \mathrm{~km}$ de plages de ponte (données du tableau 2, in Sella, 1982). On a observé de 100 à $150 \mathrm{nids}$ au cours de rapides examens aériens, en 1968 (Sella, 1982). Sur les dix à vingt nids que l'on trouverait chaque année en Israël, depuis 1980, la plupart sont des nids de Caretta (Kuller, in litt.).

\subsubsection{Plages de ponte et nombres : Chelonée franche (Chelonia mydas)}

Selon un vieux pêcheur interrogé par Sella, il y avait des nidifications sur toutes les plages sablonneuses du nord d'Israël au cours des années 1920 et 1930 (Sella, 1982). Il ne semble pas y avoir eu de vérification indépendante de cette information, qui doit être accueillie avec les précautions voulues; il ne semble pas que l'on puisse trouver, dans les documents disponibles, de renseignements sur le nidification en Israël septentrional, au cours de cette période. On peut remarquer qu'Hornell (1935), qui parle pourtant de l'ancienne pêche à la tortue, ne mentionne ni nidification de tortues ni capture sur les plages de ponte. Peut-être n'y avait-il pas (ou que peu) de nidifications de Chelonia pendant les années 20 et 30 dans les limites de ce qui est devenu Israël (voir section 3.10., Liban). Dans les années 60 , on ne trouvait que des nids isolés, à Netanya, Césarée, Atlit, Nahariya et Rosh-Hanigra (Sella, 1982). Des 10 à 20 nids signalés annuellement en Israël, au cours des années 80 , quelques-uns seulement étaient de chélonées franches (Kuller, in litt.). 


\subsubsection{Tendances des populations}

L'information qui précède suggère que le nombre de femelles de Caretta nichant chaque saison se serait abaissé de 70 dans les années 50 à un maximum de 50 et un minimum de peut-être une dizaine dans les années 60 (en supposant trois couvées chacune par saison). En 1979, on n'a signalé que deux nids et sept émergences sans nidification, sur $250 \mathrm{~km}$ de côte (Sella, 1982). Ces dernières années, seulement 10 à 15 nids (Mendelssohn, in litt.), ou 10 à 20 (Kuller, in litt.) des deux espèces auraient été aménagés sur la côte méditerranéenne d'Israël, la plupart étant de Caretta, et quelques-uns de Chelonia.

Bien que la chélonée franche ait pratiquement disparu en tant qu'espèce reproductrice en Israël, l'ignorance de l'ancienne dimension de la population ne permet pas d'apprécier l'ampleur de l'apparente diminution du nombre de nidifications.

\subsubsection{Les tortues en mer}

Comme nous l'avons dit, Caretta aurait été assez fréquente sur les côtes vers la fin du dix-neuvième siècle (Tristram, 1888, cité par Flower, 1933), et Chelonia mydas se voyait en grand nombre, chaque saison, au large de la côté septentrionale, selon Hornell (1935). Un vieux pécheur interrogé par Sella (1982) pensait que Chelonia mydas migrait vers le nord au printemps, traversant la zone de pêche au large d'Acre, pour se rendre sur la côte turque, où l'on suppose qu'elle nichait. La saison de la pêche à la tortue aurait duré d'avril à juillet, période, selon Sella (1982), de l'appariement et du nidification dans la région. Hornell (1935) mentionne que l'on voyait un grand nombre de Chelonia au large de la côte nord entre Acre et Abu Zabura (près d'Hedera), "pendant la saison chaude", lorsqu'elles $s^{\prime}$ approchaient du rivage pour se nourrir de plantes marines dans les eaux maigres (ainsi que de fruits ou déchets jetés à la mer à l'occasion de chargements). On ne sait au juste si la "saison chaude" évoquée par Hornell correspond à la période d'avril à juillet mentionnée par l'informateur de Sella. Hornell met en lumière le comportement saisonnier des tortues, suggérant que les Chelonia jadis exploitées dans les eaux palestiniennes étaient des migrantes se rendant aux sites d'alimentation et de nidification; vu l'absence de preuve définitive de l'existence passée de nombreux nidifications en Israël, il est possible que la plupart des animaux aperçus aient fait partie d'une population nichant en Turquie et qui a fait l'objet d'une forte exploitation au cours des décennies suivantes (voir section sur la Turquie).

\subsubsection{Menaces}

\section{Exploitation}

Sella (1982) attribue d'abord la forte diminution du nidification de tortues en Israël à la surexploitation, surtout dans les années 20 et 30 , mais aussi au siècle précédent, situation qui s'est aggravée depuis quelques décennies par l'extraction de sable de certaines plages de ponte, et par les perturbations en général.

Gruvel (1931) note l'existence d'un commerce international de tortues de mer depuis la Méditerranée orientale vers l'Egypte et l'Angleterre. Lortet (1883) signale avoir vu plusieurs centaines de tortues qu'il a identifiées comme des Caretta, rejetées sur le rivage d'Haïfa par une violente tempête; Sella (1982) pense qu'il pourrrait s'agir de femelles reproductrices, mais croit plus vraisemblable que ces tortues étaient rassemblées là à des fins d'exportation. 
Hornell (1935) indique que "depuis plusieurs années" à peine 200 sur les près de 2.000 chelonées franches (Chelonia mydas) capturées annuellement au large de la Palestine avaient été exportées en Egypte, et que ce commerce d'exportation avait cessé au moment où il écrivait, en raison de l'imposition, par l'Egypte, de droits de douane élevés. Cela montre que la capture des tortues sur la côte septentrionale d'Israël s'est surtout limitée aux années 20 . Ces animaux étaient capturés au moyen de seines de lin particulièrement fortes, à larges mailles (30 à $40 \mathrm{~cm}$ ), mesurant de 120 à $200 \mathrm{~m}$ de longueur et plongeant à $10 \mathrm{~m}$ de profondeur. Hornell (1935) recommandait que des efforts fussent faits pour la reprise de ce commerce, aux fins d'exportation vers Londres ou d'ouverture, localement, d'une conserverie.

Se fondant sur le témoinage verbal obtenu, dans les années 60 , d'un vieux pêcheur qui avait organisé la pêche à la tortue dans les années 20 et au début des années 30, Sella (1982) estimait qu'un minimum de 30000 tortues avaient été capturées au large de l'actuel Israël septentrional au cours de cette période. Ce qui ferait quelque 2000 tortues par an. Le pêcheur en question employait jusqu'à douze équipages, dont chacun disposait de deux bateaux et opérait avec un filet à mailles d'environ $40 \mathrm{~cm}$ au cours de la saison d'accouplement et de nidification (d'avril à juillet). En récoltant tout ce que retenait le filet, on arrivait à un maximum de quelque 600 tortues par jour, dont $90 \%$ étaient des chélonées franches. D'autres entrepreneurs, avec leurs équipages, faisaient leur propre récolte, dont on ignore l'importance.

Bien que cette exploitation commerciale ait certainement affecté la population en cause, on ne sait au juste où étaient ses plages de ponte; il semble probable qu'on prélevait sur une population nichant jadis au nord d'Israël, mais aujourd'hui pratiquement éteinte. Il est également possible que certaines des tortues capturées aient appartenu aux populations reproductrices de la côte méditerranéenne de la Turquie, et aient été prises au cours de migrations ou sur leur aire d'alimentation.

La pêche à la tortue s'est poursuivie jusque dans les années 60 , bien qu'elle ait été suspendue au cours de la deuxième guerre mondiale, et elle n'a consisté qu'en prises occasionnelles pour le marché national jusqu'à la fin des années 60 , alors qu'elle s'est complètement arrêtée (Sella, 1982). On trouve encore des tortues dans des chaluts; lorsqu'elles sont vivantes, à la remontée du filet, ces tortues sont relâchées, bien que parfois on puisse conserver la carapace à titre de curiosité (Pisanty, in litt., 28 août 1988). La fréquence des prises incidentes est inconnue. Les tortues marines sont protégées en Israël et, comme leur chair est interdite aux juifs, elles ne sont pas pêchées intentionnellement depuis quelques années (Pisanty, in litt.).

\section{Dégradation de 1'habitat}

Les principales plages de ponte de Nahariya, Rosh Hanigra et Atlit ont été sérieusement mutilées par l'extraction de sable pour la production de béton, entre 1954 et 1963. Le rivage a été dénudé jusqu'à la roche entre Nahariya et Rosh Hanigra, tandis qu'à Atlit, on a amputé de quelque $80 \mathrm{~m}$ une plage qui en mesurait initialement 120. $D^{\prime}$ autres plages ont été mutilées, de sorte qu'un nombre croissant d'émergences de tortues n'ont pas été suivies de nidification, ou que certaines couvées ont péri inondées par les eaux de la mer (Sella, 1982). L'extraction de sable s'est terminée vers 1970 , et I'on a noté 
une amélioration de l'état des plages quelque cinq ans plus tard. Bien qu'Israël ne possède pas de plages isolées, et que toutes soient le théâtre d'activités humaines (Kuller, in litt.), ces perturbations ne constituent peut-être pas une menace majeure pour l'actuelle population vestigiale.

\subsubsection{Mesures de protection}

Les tortues de mer sont des espèces protégées et la loi en interdit la capture; deux réserves naturelles englobent des plages autrefois importantes pour le nidification; un plan d'eclosiion protégée est actuellement mis en oeuvre (Sella, 1982). Entre 1979 et 1984, les couvées ont été déplacées et incubées artificiellement; étant donné le faible pourcentage d'éclosions, cette méthode n'est désormais employée que lorsque les nids risquent d'être submergés ou sont exposés à d'autres perturbations (Ashkenazi et Sofer, 1988).

\subsection{ITALIE}

\subsubsection{Observations générales}

On a signalé la caouanne (Caretta caretta), la chélonée franche (Chelonia mydas) et la tortue à cuir (Dermochelys coriacea) dans les eaux italiennes (di Palma, 1978 ; Bruno, 1978). Le nidification, partout de caouannes, est aujourd'hui très restreint. Bien que Bruno (par exemple en 1978) ait rapporté un apparent nidification de tortues à cuir, à la suite de la découverte de deux jeunes individus sur une plage de Macconi (province de $\mathrm{Gela}$ ), sur la côte méridionale de la Sicile, en septembre 1961, et d'oeufs, dans la même région, en juin 1967, d'autres auteurs (par exemple, Argano, 1979) doutent qu'il y ait eu nidification.

\subsubsection{Plages de ponte et nombres : Caretta caretta}

On ne connaît ou ne soupçonne que des nidifications de caouannes, très sporadiques et à distribution aléatoire, dans des localités dispersées des parties méridionales de l'Italie continentale (côte ionienne), de la Sardaigne, de la Sicile et de Lampedusa (Argano, 1979 ; di Palma, 1978 ; Bruno, 1978). Nous avons des preuves (par exemple des collections de musée de jeunes récemment éclos) que le nidification était jadis plus répandu en Italie continentale, et que les nombres étaient plus grands en certains lieux, y compris Lampedusa et la Sicile. Il n'y a cependant aucune indication qu'il y ait jamais eu de nidification de quelque importance.

La nidification est aujourd'hui extrêmement rare et irrégulière, et l'on n'a signalé que quelques nids isolés au cours des dernières années. Lampedusa semble offrir la seule plage de ponte régulière connue, mais on $n^{\prime} y$ trouve qu'un très petit nombre de nids chaque année.

\subsubsection{Italie continentale}

On a signalé des nidifications dans le passé sur les côtes ioniennes et apuliennes de l'Italie méridionale, mais la documentation fait défaut et nous n'avons aucun renseignement récent (Argano, 1979). En sept sites récemment examinés sur la côte ionienne, on $n^{\prime}$ a trouvé aucune trace de nidification (Argano et Cocco, 1988). 


\subsubsection{Sardaigne}

On a signalé naguère des nidifications sur la côte occidentale, mais on manque de documents, et il $n^{\prime} y$ a pas d'information récente (Argano, 1979). On soupçonne, surtout en raison de la fréquence des prises de caouannes près de la côte, qu'il peut y avoir des nidifications autour du golfe d'Orosei, sur la côte orientale (Voesenek et van Rooy, 1984).

\subsubsection{Sicile}

Bien qu'Argano (1979) ait considéré que la côte méridionale de la Sicile - et en particulier la plage d'Eraclea-Siculiana, à l'ouest d'Agrigente, et celle de Macconi, à l'est de Gela - soit le secteur italien le plus intéressant pour un nidification éventuel de tortues, il n'a pu trouver aucune empreinte, même après plusieurs jours de recherche sur place.

Des examens intensifs plus récents sur la côte méridionale de Sicile, de Mazara del Vallo jusqu'à Gela, vers l'est, n'ont apporté le témoignage que de nidifications occasionnels et isolés (Argano et Cocco, 1988). La visite de nombreuses plages sur les côtes nord, est et sud, en 1985, 1986 et 1988, n'a révélé aucun nidification, bien qu'on ait partout rapporté la présence, dans le passé, de nidifications de caouannes (Gramentz, in litt., 17 octobre 1988). Il y avait des nids à Linosa jusqu'à il y a une dizaine d'années (di Palma, in litt., 27 septembre 1982).

\subsubsection{Lampedusa}

On ne constate que quelques nidifications chaque année sur la plage de Conigli, à Lampedusa (Gramentz, 1986a). Il existe des témoignages d'une diminution du nombre de femelles nichant à Lampedusa, depuis la décennie 1940-1950, alors qu'un grand nombre de tortues nichaient, dit-on, sur toutes les plages de l'ille (Conigli, Cala Croce, Cala Guitgia et Cala Francese), jusqu'à la présente décennie, au cours de laquelle le nidification a pratiquement disparu (Argano, 1979 ; Gramentz, 1986a).

\subsubsection{Tendances de la population}

Dans l'ensemble, les tortues marines semblent diminuer en nombre, bien qu'on en aperçoive plus ou moins fréquemment dans les eaux italiennes; mais la nidification (Caretta) a pratiquement disparu, tant en Italie continentale que dans les iles (Argano et Cocco, 1988). Nous ne possédons cependant aucun document historique fiable permettant de mesurer la gravité du déclin de la population; le nidification semble avoir été plus étendu dans le passé, et peut-être moins rare, mais il est impossible de l'évaluer en l'absence de documentation.

\subsubsection{Les tortues en mer}

Il y a de nombreux spécimens de Caretta dans les collections de musée, et l'espèce est souvent signalée dans des documents provenant de nombreuses localités de toutes les côtes italiennes (Bruno, 1978 ; Capocaccia, 1966), y compris les îles Pelagie, Egadi, Eolie et la Sicile (di Palma, 1978). On trouve bon nombre de Caretta s'alimentant dans les eaux italiennes méridionales, surtout autour de la Sicile, le golfe de Taranto et la côte de Calabria (De Metrio et Megalofonou, 1988). 
Argano (1979) cite une information qu'il a reçue selon laquelle un vaste rassemblement de tortues a été observé en Mer tyrrhénienne, en juillet 1960; mais la signification de ce rassemblement reste inconnue.

Huit des quelques 600 caouannes baguées au sud-est de l'Italie, ont été recapturées : 3 dans l'Adriatique (Italie et Yougoslavie du Nord, Albanie), 2 au large de la Tunisie (golfe de Gabès, golfe de Tunis), 1 au centre-ouest de l'Italie, 1 à Malte et la dernière à l'ouest du Péloponnèse (Cocco, comm. pers., janvier 1988; Argano et Cocco, 1988).

On a signalé récemment (Manzella et coll., 1988) un retour de bague international sur longue distance d'une importance exceptionnelle car il apporte des détails sur le premier cas particulier de tortue de l'Atlantique migrant dans le bassin méditerranéen (et témoigne de la longue survie d'une tortue marine ayant bénéficié d'un départ protégé). Cette tortue, une caouanne, a été prise à son éclosion dans un nid d'une plage de Floride, en 1981. Elle a été protégée à Galveston et lâchée, à un an, de l'île de Padre, en juin 1982. Au moment du lâché, elle pesait $546,2 \mathrm{~g}$ et sa carapace mesurait $14,4 \mathrm{~cm}$ de longueur et $12,1 \mathrm{~cm}$ de largeur (Schroeder, in litt., 12 décembre 1386, à R. Argano). Au moment de la recapture, quatre ans plus tard août 1986 - elle était en bon état, pesait $9 \mathrm{~kg}$, avec une carapace d'une longueur de $43,5 \mathrm{~cm}$ et d'une largeur de $41 \mathrm{~cm}$ (on ne sait s'il s'agit de ligne droite ou courbe) (Manzella et coll., 1988). Elle s'était prise à un aplet de pêche à l'espadon, à quelque dix milles marins au sud de Santa Maria Leuca (sud-est de l'Italie, à l'entrée de l'Adriatique).

Les caouannes sont capturées accidentellement, en petit nombre, mais tout au long de l'année, par des pêcheurs du centre-nord de l'Adriatique (à la différence des eaux adriatiques et ionniennes du sud, où les prises sont nombreuses, mais rares en hiver). Les tortues prises pendant les mois d'hiver dans le centre-nord de l'Adriatique sont dans un état de torpeur apparente sur des fonds vaseux; il pourrait s'agir d'une région importante comme terrain d'hivernage ou d'hibernation (Argano et Cocco, 1988).

Des caouannes baguées, alors qu'elles richaient en Grèce, ont été retrouvées dans l'Adriatique septentrionale, dans le golfe de Tarente et sur le côtes de la Sardaigne (Margaritoulis, 1988a).

\subsubsection{Menaces}

\section{Captures accidentelles}

Il y a des prises assez nombreuses, surtout incidentes, dans l'Adriatique et autour de la Sicile et de la Sardaigne, et des caouannes mortes sont rejetées occasionnellement, mais régulièrement sur des plages italiennes du sud. Certaines prises de caouannes ne sont pas incidentes; Di Palma (1978) a noté qu'il se faisait une pêche indépendante dans les iles éoliennes (Lipari), au nord de la Sicile, et qu'on pouvait estimer les prises entre 500 et 600 tortues annuellement (sans doute uniquement ou presque uniquement des caouannes). On ne connait pas l'actuelle situation de la pêche dans ces îles. Pour la période 1978-81, on a estimé que de 250 à 1.000 Caretta étaient prises chaque année dans le golfe de Tarente, principalement à l'occasion de la pêche au thon blanc (De Metrio et coll., 1983). Le nombre de prises signalées pour 1982-1986 était inférieur mais ces données ne paraissent guère fiables (De Metrio et Megalofonou, 1988). Bien que la plupart des prises de tortues soient accidentelles, certains des 
animaux capturés sont utilisés dans plusieurs collectivités côtières, où on les prépare selon des recettes locales (Argano et Cocco, 1988). La chair de tortue est considérée comme un mets délicat et reste très recherchée le long de la côte ionienne des Pouilles (De Metrio et Megalofonou, 1988).

Un projet récent soutenu par la section italienne du Fonds mondial pour la nature a permis de capturer, de marquer et de relacher quelque 600 caouannes, dont la plupart ont été reprises par des pêcheurs, généralement accrochées à des aplets pour espadon (Argano et Cocco, 1988). On a ainsi retrouvé plus de 375 tortues dans la seule année 1986. L'activité s'est concentrée sur la côte apulienne, au sud-est de l'Italie, où se sont établies de bonnes relations avec les collectivités de pêcheurs locales. La plupart des prises ont lieu entre mai et octobre, avec un maximum en juillet-août (lorsque la mer est le plus calme et que les pêcheurs sortent presque tous les jours).

Le long de la seule côte ionienne de Calabre, chaque chalût capture de trois à cinquante Caretta par sortie en mer, ce qui représente une prise annuelle totale de 16.000 dans cette zone (De Metrio et Megalofonou, 1988). Ce total très élevé prend probablement en compte les tortues capturées à plusieurs reprises; néanmoins, le taux général de mortalité atteint probablement au minimum quelques milliers.

On sait qu'un grand nombre de caouannes sont prises accidentellement par des pêcheurs dans les eaux siciliennes (Cocco, comm. pers., janvier 1988), et autour de la Sardaigne, où les tortues peuvent être vendues à des restaurants, et les carapaces à des touristes (Ballasina, comm. pers., janvier 1988). Les pêcheurs $n^{\prime}$ ont pas coopéré avec les spécialistes de la tortue, et nous avons peu d'information sur la dimension des prises dans ces régions. Un spécialiste qui connaît bien les lieux (Gramentz, in litt., 17 octobre 1988) a estimé que plus de 5.000 tortues sont prises chaque année par les pêcheurs siciliens, et qu'au moins 1.000 de ce nombre sont tuées; que 150 à 300 tortues sont tuées autour de Lampedusa, sur une prise totale de peut-être trois fois ce nombre. Bien que les tortues accrochées aux aplets soient généralement rejetées à l'eau après coupure de l'aplet, on les décapite parfois pour récupérer l'hameçon (Gramentz, in litt., 17 octobre 1988).

On ne connaît pas le taux de mortalité des tortues prises aux aplets mais on le suppose élevé, la mort étant causée par la privation de nourriture ou le saignement interne de l'intestin à l'endroit de l'occlusion provoquée par l'hameçon. L'une des études évalue à près de $30 \%$ le taux de mortalité des tortues prises dans des chaluts; la plupart d'entre elles sont de petite taille ou de taille moyenne.

\section{Dégradation de I'habitat}

Les aménagements pour le tourisme, l'industrie et l'agriculture ont sérieusement mutilé un très grand nombre de plages, y compris celles qui auraient servi jadis aux tortues reproductrices. Par exemple, la plage de

Macconi, près de Gela, en Sicile méridionale, n'est probablement plus propre à recevoir des tortues reproductrices en raison de la construction de raffineries de pétrole dans le voisinage, de serres et de logements touristiques à proximité du rivage (Argano, 1979; Bruno, in litt., 1986). Les caouannes (apparemment reproductrices) que l'on trouvait à Imera, près de

Buonfornello, sur la côte nord sicilienne, n'ont pas été revues depuis environ 1970; il y avait là une plage d'une centaine de mètres de largeur, mais l'extraction de sable pour la construction et la récupération de marais 1 'a réduite à $12-15$ mètres (Gramentz, in litt.). 


\section{Pollution}

La pollution nuit également aux tortues dans les eaux italiennes. Une caouanne de $40 \mathrm{~cm}$ a été trouvée dérivant à environ 200 mètres au large de la côte nord de Lampedusa, partiellement immobilisée par des rebuts pétroliers: elle a été nettoyée et relâchée (Gramentz, 1986b). Selon Ballasina et Frisenda (comm. pers., décembre 1988), des articles récents dans la presse italienne signalent que des centaines de Caretta adultes ont été trouvées mortes sur les plages du sud de I'Italie. Selon ces reportages, la mort des tortues serait imputable à la pollution provenant de l'industrie agrochimique. Toujours selon Ballasina et Frisenda, cette mortalité serait un phénomène courant ces dernières années. Margaritoulis (comm. pers., septembre 1987) signale qu'environ 200 tortues se sont échouées sur la côte adriatique du sud de l'Italie en 1986; on suppose que la pollution est la principale cause de mortalité.

\subsubsection{Mesures de protection}

Les tortues marines sont expressément protégées en juridiction italienne par un texte du 21 mai 1980. La pêche, la capture, le transport et le commerce des tortues sont interdits. Le "Progetto Tartarughe Marine", lancé à l'Université de Rome et soutenu par le Fonds mondial pour la nature-Italie, est mis en oeuvre en Sicile et en Italie du sud-est depuis 1986. Il comprend des visites de plages ainsi que la collecte, le marquage et la relâche de caouannes prises par des pêcheurs.

\subsection{LIBAN}

A la fin du 19 e siècle, Caretta aurait été assez commune au large des côtes de la grande région palestinienne, et l'on a vu des pêcheurs en ramener à Sidon (Tristram, 1888, cité par Flower, 1933). Selon Lortet (1883), des tortues marines - qu'il identifia comme étant des Caretta caretta - étaient aperçues passer fréquemment le long des côtes du Levant, où elles broutaient les algues le long des rives rocheuses, et flottaient par groupes près de la surface des eaux claires du sud de la "Syrie" (correspondant à l'actuel Liban). Il signale avoir vu de très grands spécimens autour de l'embouchure du Nahr el Kelb (entre Jounie et Beyrouth), autour de Ras el Abiad (Cap blanc, entre Tyr et Sidon), ainsi que dans la région de Tyr. Quelques décennies plus tard, Gruvel (1931) notait que Caretta était présente le long de la côte libanaise, mais moins nombreuse que plus au nord, dans le golfe d'Iskenderun. Lortet (1887) estimait que les individus de grande taille avaient entre $1,5 \mathrm{~m}$ et $2 \mathrm{~m}$ de longueur, ce qui semble tout à fait exagéré (même en comptant la tête en plus de la carapace; l'exagération serait moins grande s'il s'agissait de Chelonia plutôt que de Caretta.

On ne sait dans quelle mesure les tortues marines nichaient sur la côte libanaise dans le passé. Lortet (1883) note avoir capturé un couple de Caretta apparié sur la plage de Tyr, et il précise, en 1887, que cette prise a eu lieu au cours de la nuit, sur une plage de sable s'étendant au sud de Tyr (peut-être dans les actuelles limites d'Israë1). Il ajoute que les chameliers locaux, qui se sont empressés de trancher la gorge des tortues, trouvaient souvent des couples dans cette situation. Les pêcheurs de Tyr indiquèrent (Lortet, 1887) que les couvées n'éclosaient qu'en juillet (ce qui suggère le nidification en mai) et que les jeunes couraient immédiatement à la mer. Ces observations semblent confirmer qu'il y avait jadis des nidifications, bien 
que la découverte de couples appariés sur la plage paraisse anormale. On ne constate plus de nidifications aujourd'hui et des tortues ne sont que très rarement signalées dans les eaux libanaises (Kouyoumjian, H.H., in litt., 16 septembre 1988). Bien qu'il existe une législation générale pour la protection de la vie sauvage, elle n'est pas appliquée actuellement, et il $n^{\prime} y$ a pas de texte se rapportant en particulier aux tortues marines (Kouyoumjian, H.H., in litt., 16 septembre 1988 ).

\subsection{LIBYE}

On sait qu'une petite population de Caretta niche sur des plages du Parc national Kouf (Cyrénaïque) (Schleich, 1984); on a compté de 60 à 65 nids pour chacune de deux saisons récentes, ce qui représentait peut-être quelque 20 à 30 femelles par an. Les tortues et leurs nids sont souvent victimes des chacals, et les perturbations d'origine humaine vont croissant. Des empreintes de Caretta auraient été vues sur des plages voisines d'Al Quarahbulli (= Garaboli ?), à quelque $60 \mathrm{~km}$ à l'est de Tripoli (Bruno, comm. pers., cité par Fretey, 1987). Il est possible $q^{\prime}$ 'il y ait des nidifications en d'autres lieux, mais nous ne disposons d'aucune information récente. Il y a des indications de l'existence d'au moins une zone majeure de nidification, et il y a d'importants lits de plantes marines comparables à ceux du golfe de Gabès (Jeudy de Grissac, comm. pers., octobre 1989).

Une caouanne femelle, baguée alors qu'elle nichait en Grèce, a été recapturée au large de la Libye (Margaritoulis, 1988a).

\subsection{MALTE}

\subsubsection{Observations générales}

On signale cinq espèces dans les eaux maltaises: Caretta caretta, Dermochelys coriacea, Chelonia mydas, Eretmochelys imbricata et Lepidochelys kempi (Brongersma et Carr, 1983). La caouanne demeure relativement commune, Dermochelys a été signalée en plusieurs occasions, et Chelonia, Eretmochelys et Lepidochelys ne sont mentionnées qu'une fois (Brongersma et Carr, 1983; Gramentz, in litt., 1988).

\subsubsection{Plages de ponte et nombres: la caouanne (Caretta caretta)}

Bien que la carette ait niché jadis dans la baie de Ramla il-Hamra, à Gozo, on ne l'y trouve plus, à la suite des constructions et des perturbations touristiques; les derniers nidifications signalés datent du début des années 1940 (Balzan, in litt., 22 août 1988; Lanfranco, in litt., juillet 1988; Baldacchino, in litt., 11 août 1988). Nous n'avons pas de renseignements sur la dimension des anciennes populations reproductrices. Despott (1915) signale que Caretta "aurait niché sur des plages non fréquentées, notamment à Gozo, ce qui suggère que le nidification était probablement mineur, peut-être irrégulier, et que Gozo n'était pas le seul lieu de ponte. 


\subsubsection{Les tortues en mer}

Selon Despott (1915), Caretta était très fréquent autour de Malte. Un assez grand nombre de Caretta s'alimentent dans les eaux maltaises; surtout de juin à septembre, ce qui coïncide avec les activités maximales de pêche à l'espadon, au thon et au dauphin (Balzan, in litt.). Il n'est pas rare de voir des tortues se chauffer au soleil, en surface ou sous des feuilles de palmier employées comme flotteurs de pêche (Balzan, in litt.). Des caouannes baguées à Zante (Grèce) ont été recapturées dans les eaux maltaises (Margaritoulis, comm. pers.).

\subsubsection{Menaces}

\section{Exploitation, prise accidentelle}

Au début du siècle (Despott, 1915), on capturait un grand nombre de Caretta en mer, d'août à novembre, et l'espèce servait de nourriture à la majorité de la population. De nos jours, les pêcheurs prennent nombre de Caretta tant volontairement qu'incic'emment, à l'occasion de pêche au thon ou à l'espadon, avec aplets ou filets. On estime que de 1.000 à 2.000 d'entre elles sont ainsi prises chaque année (Balzan, "Zghazagh ghall-Ambjent", in litt.). Gramentz (in litt., 17 octobre 1988) a estimé que de 2.000 à 3.000 Caretta se prennent à des aplets au cours de la saison de l'espadon (printempsété) dans le voisinage des îles maltaises, et que de 500 à 600 d'entre elles sont tuées chaque année. Dans un seul village, Gramentz a acheté, bagué et relâché 101 caouannes au cours de l'été. Si ces diverses estimations sont exactes, la mortalité est de 15 à $50 \%$ de la prise totale. Les pêcheurs capturent les tortues qui se chauffent au soleil au moyen de gros hameçons. Les prises incidentes dans des filets sont en augmentation, avec l'emploi croissant de chaluts (Balzan, in litt.). Bien que l'on rejette généralement à la mer les tortues prises aux aplets, en coupant la corde, on les décapite parfois pour récupérer l'hameçon (Gramentz, in litt.).

Les tortues capturées servent principalement à l'alimentation, mais on en utilise aussi la carapace. On les garde généralement en vie jusqu'à la vente, pour les tuer alors par décapitation. Elles valent de 3 à 8 livres (de 9 à 24 \$US), selon leur taille; la plupart des caouannes capturées pèsent de 40 à $60 \mathrm{~kg}$, bien qu'on en trouve de plus petites et que certaines aillent jusqu'à $75 \mathrm{~kg}$. La chair de tortue est souvent consommée dans les foyers, mais plus rarement dans les restaurants (Balzan, in litt.). On vend les carapaces comme objet ornemental ou souvenir touristique (Lanfranco, in litt.; Cassar, in litt., 8 août 1988). Flower (1933) signale sa surprise de se voir offrir de la soupe à la tortue préparée avec Caretta à l'occasion de deux dîners à l'ancien palais du Gouverneur.

\section{Pollution}

Certaines des caouannes trouvées dans les eaux maltaises étaient polluées de goudron (Gramentz, 1986b) et avaient des matières plastiques et métalliques dans leurs boyaux (Gramentz, 1988). Sur 99 Caretta examinées en 1986 (après capture accidentelle par des aplets pour espadon), 20 étaient contaminées, dont 17 par du pétrole brut, et quelques-unes par des rebuts plastiques et métalliques (Gramentz, 1988). La caouanne mange généralement des mollusques, des crustacés et du poisson; les objets plastiques trouvés dans l'intestin ou les excréments de tortues examinées étaient tous transparents ou 
blancs, ce qui suggère que les tortues pouvaient les avoir ingérés en les prenant pour des méduses (Gramentz, 1988). On ne connaît pas avec précision les effets de la pollution pétrolière, bien que de petits individus puissent être immobilisés ou épuisés par une contamination lourde (Gramentz, 1988). Certaines tortues ne présentaient aucun signe extérieur de pollution pétrolière, mais on en a constaté la contamination par l'examen de la cavité buccale ou des excréments.

\subsubsection{Mesures de protection}

Les groupements locaux de protection, y compris Zghazagh ghall-Ambjent et la Société pour l'étude et la protection de la nature, ont fait campagne pour la protection juridique des tortues de mer à Malte, et pour la réduction de l'actuelle pêche à la tortue. Selon Lanfranco (in litt.), la réaction du Gouvernement est encourageante, et le ministre de l'Environnement a indiqué que des mesures seraient prises (Balzan, in litt.). On n'a pas retenu une proposition de protection de la baie de Ramla. Ni les tortues marines ni leurs habitats ne sont protégés par la législation.

\subsection{MONACO}

La zone de la plage est de construction artificielle récente, et il $n^{\prime} y$ a pas de ponte; on ne possède aucune information sur la présence de tortues dans les eaux adjacentes.

\subsection{MAROC}

\subsubsection{Lieux et nombres de nidifications}

Nous n'avons aucune confirmation de nidifications de tortues marines sur la côte méditerranéenne du Maroc, et il semble très improbable qu'il y ait des nidifications importants. Bien qu'une source suggère que Caretta caretta et Chelonia mydas nicheraient sur la côte méditerranéenne du Maroc, d'avril à septembre et de juillet à novembre, respectivement (Aouab Taha et Moumni Amina, in litt. 4 juillet 1988), ce fait n'est pas confirmé par Laurent (1989a). Celui-ci a enquêté sur un échantillon de plages, a interrogé des pêcheurs et d'autres personnes, et examiné des documents remontant au milieu du 19e siècle. Il n'a observé aucun signe d'activités de nidification lors de ses enquêtes sur les plages, en juin et juillet. Parmi de nombreuses personnes interrogées, une seulement se rappelait avoir vu une présumée empreinte de tortue ( 15 ans plus tôt, à Ras el Ma). Laurent n'a trouvé aucun témoignage de première main de nidification dans les documents anciens ou récents, ni aucun rapport documentaire. Bien qu'il existe des plages de sable convenables, une température marine relativement basse associée au régime de courant de la Méditerranée occidentale empêche probablement le nidification des tortues, comme c'est peut-être aussi le cas pour l'Espagne et l'Algérie.

\subsubsection{Menaces}

Laurent (1989a) estimait, après des entretiens avec de nombreux pêcheurs, que plus de 3.000 Caretta sont capturées (y compris les recapturées) par les pêcheurs à l'aplet à espadon ayant leur port d'attache au Maroc. De nombreuses tortues sont relâchées en mer, mais il existe une assez importante vente locale de carapaces à des touristes, ainsi que de chair et de carapaces à des résidants 
espagnols du Maroc; certaines carapaces sont exportées vers l'Espagne (ce commerce international mineur semble unique entre pays méditerranéens). La plupart des prises accidentelles semblent associées avec les ports de Nador et Al Hoceima, dans le Maroc central, sur la côte méridionale de la Mer d'Alboran (Laurent, 1989a).

\subsubsection{Mesures de protection}

Il n'y a pas de législation de protection en vigueur (Laurent, 1989a).

\subsection{ESPAGNE}

\subsubsection{Observations générales}

La plupart des renseignements intéressent les îles Baléares, dans le voisinage desquelles on voit des Caretta en grand nombre, de dimension principalement adulte ou quasi adulte. Nous n'avons pas de données sur les territoires espagnols d'Afrique du Nord (Ceuta, Mellila). On ne connaît pas de nidification récent en Espagne continentale, et rien n'indique que les tortues auraient niché en grand nombre dans le passé. On a signalé des nidifications tout à fait occasionnels, sur la côte méditerranéenne de la péninsule, au cours des années 1920, et l'on trouve dans les musées un petit nombre de spécimens de très jeunes Caretta. Mais les températures du sable peuvent être généralement trop basses pour assurer des conditions d'incubation optimales, et une construction littorale intense empêche aujourd'hui toute nidification importante (J. Mayol, comm. pers., janvier 1988). Une femelle Caretta, apparemment en bonne santé, a été découverte sur une plage près de Malaga le 29 avril 1986 (Caminas, 1988), ce qui suppose qu'il y a occasionnellement des tentatives de nidification.

On dispose de peu d'information sur les territoires espagnols d'Afrique du Nord (Ceuta, Melilla). On capture des tortues pour les consommer, apparemment en petit nombre, et il se fait un commerce mineur de carapaces (Heredia, B., comm. pers., octobre 1989).

\subsubsection{Les tortues en mer}

On trouve dans les eaux espagnoles un grand nombre de tortues - principalement Caretta - qui migrent ou viennent s'y alimenter, et l'on dit que les pêcheurs sont très familiers de Dermochelys (Mayol et Castello Mas, 1983).

\subsubsection{Menaces}

\section{Capture accidentelle}

On compte beaucoup de captures accidentelles près des Baléares, par les aplets tendus pour le requin et l'espadon (Mayol et Castello Mas, 1983). Certains équipages ont leur base aux Baléares, mais la plupart sont d'Espagne continentale et rapportent leurs prises à la péninsule; ils pêchent aux îles entre février et septembre, ils vont du côté de Gibraltar et de l'Atlantique à d'autres époques de I'année. 
Chaque aplet peut compter de 1.000 à 1.500 hameçons, armés de tassergals ou de calmars, et chaque équipage prend accidentellement, tous les jours, de 3 à 10 tortues (moyenne de 5,7 ). Tous les pêcheurs $s^{\prime}$ accordent à dire que Caretta se rencontre couramment à partir de la fin mai mais est devenue plus rare en été. La plupart d'entre elles sont capturées de mai à août, ce qui correspond en grande partie à la période de pêche la plus intense (Caminas, 1988). Comme environ 80 bateaux ont fait la pêche au cours des dernières années, on peut estimer la prise incidente totale de tortues de 16.000 à 17.000 par saison (Mayol et Castello Mas, 1983). Des recherches récentes de l'Institut océanographique espagnol confirment que l'on prend de 17.000 à 20.000 tortues marines dans la région des Baléares. Ce chiffre considérable comprend une proportion substantielle mais non exactement connue de recaptures (Sagarminaga, comm. pers. 1988), et la mortalité effective qui en découle est incertaine.

Il y a, dit-on, de nombreuses captures accidentelles de tortues prises à des aplets de pêche à l'espadon dans la zone d'Alboran (Caminas, 1988). Près de la moitié des espadons pêchés par les Espagnols en Méditerranée sont déchargés à Alicante, et se fondant sur des données recueillies dans ce port, Caminas (1988) estime que 7478 et 8389 Caretta ont été capturées et relâchées dans la région en 1986 et 1987 (il est difficile de dire si ces estimations concernent la zone d'Alboran, la région des Baléares ou les deux).

Les pêcheurs libèrent généralement les tortues en coupant l'hameçon auquel elles sont accrochées, de sorte qu'elles restent souvent avec une partie de crochet enfouie dans la bouche ou la gorge. On pense que la survie dépend de la gravité de la blessure. Il semble que les petits hameçons causent le plus de mortalité, et les pêcheurs ont tendance à les utiliser en raison du fait que l'espadon est surexploité (Mayol, comm. pers., janvier 1988). Selon une expérience de J. Mas (rapportée par Caminas, 1988), sur six Caretta hameçonnées, une seule est morte; les autres ont pu rejeter l'hameçon et être libérées vivantes. Les tortues non libérées sont vendues à des restaurants, sur des marchés ou à des touristes, sous forme de carapaces apprêtées (les prix vont de 300 à 1.500 pesetas) (Mayol et Castello Mas, 1983).

\subsubsection{Mesures de protection}

On poursuit l'étude des problèmes des prises incidentes dans la zone des Baléares (projet du Fonds mondial pour la nature, 3803, Espagne), notamment par des collectes de données et un examen des moyens qu'il faudrait mettre en oeuvre pour réduire les prises incidentes et accroitre les chances de survie des tortues hameçonnées (Mayol, comm. pers. 1987). Les tortues marines sont expressément protégées en Espagne en vertu du décret 3181 .

\subsubsection{Divers}

La plupart des caouannes prises à des aplets autour des Baléares ont des carapaces de 30 à $65 \mathrm{~cm}$, ligne droite, une nette majorité ayant entre 45 et $55 \mathrm{~cm}$ (moyenne de 49,5, pour des longueurs allant de 20 à $75 \mathrm{~cm}$ ) (Mayol et Castello Mas, 1983). La mesure moyenne courbe de la carapace est de $52,6 \mathrm{~cm}$. L'échantilion comprend donc surtout des tortues presque adultes, certaines étant loin de la maturité, et quelques-unes l'ayant probablement atteinte. 


\subsection{SYRIE}

Nous $n^{\prime}$ avons que peu de renseignements. Lortet $(1883,1887)$ rapporte que certaines tortues marines, qu'il a reconnues comme des Caretta caretta, se voient assez ou très fréquemment le long des côtes du Levant, où elles broutent les algues des rives rocheuses. Geldiay indique (comm. pers., 2.9.87) qu'on lui a informé qu'on peut voir un grand nombre de tortues dans les eaux syriennes, et $l^{\prime}$ on observe des nidifications importants. Un vieux pêcheur, qui a bien connu les tortues de Méditerranée orientale dans les années vingt et trente (voir section sur Israël), et s'est entretenu avec Sella dans les années soixante (Sella 1982), n'a eu connaissance d'aucun nidification en Syrie (voir section 3.8., Israël). Les animaux étaient fréquemment capturés et exportés vers l'Egypte jusqu'en 1960 (quelque 200 à 250 par mois pour cette année-là); on en capture beaucoup moins aujourd'hui (Laurent, 1989b).

\subsection{TUNISIE}

\subsubsection{Observations générales}

Baccar (in litt., 17 août 1988) écrit que quatre espèces ont été signalées dans les eaux tunisiennes: Caretta caretta, Chelonia mydas, Dermochelys coriacea et Eretmochelys imbricata (aucun document disponible). Des témoignages récents ne confirment la présence régulière que de Caretta, Dermochelys et Chelonia n'étant qu'occasionnelles (Lescure, in litt., 15 septembre 1988). Des signalements de Caretta cités par Loveridge et Williams (1957) intéressent les marchés de Bizerte, Gabès, Tunis et Sfax.

On n'a que peu d'information sur le nidification, et il n'y a guère de documents sur la ponte dans le passé. Selon Argano (1979), la côte septentrionale de la Tunisie ne présente aucun intérêt particulier pour le nidification des tortues, mais la côte orientale offre les plus importantes plages de ponte de tout le secteur occidental africain de la Méditerranée. Cette estimation semble exacte, dans la mesure où il n'y a pas de nidification régulier en Algérie ni au Maroc (Laurent 1989a), mais des nidifications épars de Caretta en Tunisie (Laurent et coll., sous presse).

\subsubsection{Lieux et nombres de nidifications : Caretta caretta}

La caouanne est la seule espèce à nicher en Tunisie. Les résultats d'un récent examen des plages du Nord et du Sud-Est (Laurent et coll., sous presse) indiquent que les nidifications sont extrêmement rares (lé secteur nord-est de la côte n'a pas encore été inspecté). $\mathrm{L}^{\prime}$ examen de 900 kilomètres de côtes comprenant $40 \%$ de la longueur de plages disponible n'a relevé qu'un seul nid dont la couvée avait récemment émergé (le ler septembre, ce qui dénote un nidification fin juin ou début juillet), et ailleurs un nouveau-né survivant. On n'a observé aucune empreinte révélatrice de nidification. On a en revanche constaté directement des nidifications au bord de la plus grande des Iles Kuriates (à 15 kilomètres au large de la côte voisine de Monastir), et sur la plage continentale entre Ras Dimass et Mahdin ( 25 kilomètres au sud de Monastir). Des témoignages de pêcheurs locaux suggèrent qu'il y avait des nidifications réguliers aux îles Kuriates, peut-être de deux à cinq nids chaque année, et peut-être également quelques-uns sur la plage continentale. Les gens du pays ont également suggéré qu'il y avait des nidifications sur la plage sise entre El Bibane et la frontière libyenne, à 
Ghannouch et à Thapsus (ou à l'Ile Ras Dimass). Plusieurs plages étaient considérées comme des lieux de nidification éventuels, mais $n$ 'ont pas été examinées. Selon Baccar (in litt., 17 août 1988), Caretta niche au Cap Serra (Tunisie septentrionale) et dans le golfe de Gabès (Sud tunisien). Gramentz (in litt., 26 juillet 1988) rapporte qu'il y a des nidifications près de Nabeul dans le golfe d'Hammamet.

Selon Laurent et coll. (sous presse), l'actuelle population reproductrice de Caretta, apparemment tout à fait mineure, était certainement plus dense par le passé, sans que l'on puisse cependant en apporter la preuve directe. Le déclin supposé est attribué à la capture de tortues, à l'enlèvement des oeufs ou des femelles sur les plages de ponte; et à l'accroissement du développement touristique au cours des 15 dernières années (Laurent et coll., sous presse).

\subsubsection{Les tortues en mer}

La tortue verte Chelonia mydas n'aurait pas été rare dans le Sud tunisien au cours des premières décennies du siècle (sources citées dans Laurent et coll. sous presse), mais elle l'est

aujourd'hui. Trois seulement des 120 carapaces examinées au cours de l'enquête de Laurent étaient des Chelonia mydas; les nombreux pêcheurs interrogés ne semblaient pas connaître l'espèce, bien que les marchands de poisson du marché de Bab Jebli, à Sfax, en eussent été familiers, et eussent déclaré en recevoir un spécimen tous les deux ou trois ans (Laurent et coll. sous presse). L'apparente diminution en nombre de cette espèce dans les eaux tunisiennes est probablement le simple résultat de la dégradation des populations reproductrices en Méditerranée orientale.

L'un des rares spécimens récents de Chelonia a été signalé à Sfax (mars 1987), et avait été bagué à Chypre (Laurent et coll. sous presse); il faisait vraisemblablement partie de la petite population nichant dans la région de Lara, où a été mis en vigueur le programme de protection des tortues de Chypre (voir section sur Chypre).

Laurent et coll. (sous presse) signalent que Caretta se trouve en nombre relativement élevé près des côtes tunisiennes. On sait qu'elles sont nombreuses dans le golfe de Gabès, vaste zone d'eaux maigres abritant l'un des plus grands lits de plantes marines de la Méditerranée. Des travaux plus anciens suggèrent que les animaux étaient plus nombreux au printemps: il s'agissait peut-être d'une population nichant sur les plages tunisiennes. La plupart des tortues sont maintenant capturées par des chaluts au cours de l'hiver. Il se peut qu'il y ait moins de tortues au printemps à la suite du déclin d'une ancienne population reproductrice tunisienne; ou peut-être découvre-t-on davantage de tortues en hiver en raison du fait que le chalutage de fond est largement pratiqué depuis les années 60 .

La majorité des retours de bagues de la population reproductrice grecque de Caretta se font dans le golfe de Gabès, en hiver (Margaritoulis, comm. pers., 2 septembre 1987). Des caouannes capturées en mer, baguées et relâchées le long des côtes sud-est de l'Italie, ont également été retrouvées dans cette zone, ainsi qu'en Tunisie septentrionale (Argano et Cocco, 1988). Le grand nombre de Caretta aperçues, et le témoignage des retours de bagues, confirment que le golfe de Gabès est un habitat essentiel pour la Caretta méditerranéenne, apparemment surtout hors nidification, au cours de I'hiver. 


\subsubsection{Menaces}

\section{Capture accidentelle}

Caretta est évidemment menacée par l'importante consommation qu'en font principalement les foyers, mais une large proportion des animaux capturés sert à confectionner des souvenirs pour touristes. Argano et Baldari (1983) estiment à 1500 les captures annuelles. La récente enquête de Laurent et de ses collègues (Laurent et coll., sous presse) indique qu'environ 4.000 à 5.500 Caretta sont capturées dans le golfe de Gabès à l'occasion d'activités de pềche, principalement par la flotte de chalutiers de fond opérant au large de Sfax pendant l'hiver. Peut-être de 20 à $30 \%$ de la capture totale de tortues est-elle le fait d'autres activités de pêche employant des aplets à espadon, des seines à thon et d'autres filets ou lignes ílaurent et coll., sous presse). On prend des oeufs et, à l'occasion, des femelles reproductrices sur les plages de ponte.

Les tortues capturées accidentellement au chalut sont généralement vivantes à l'embarquement, mais elles sont toutes retenues pour l'alimentation (Laurent et coll., sous presse). La chair de tortue fait traditionnellement partie du régime alimentaire de nombreuses villes et villages du sud-est de la Tunisie; elle est meilleur marché que le poisson et achetée surtout par le secteur le plus pauvre de la population (Laurent et coll., sous presse). On considère qu'elle possède une haute valeur thérapeutique (Lanteri, 1982). L'huile de tortue est parfois employée en médecine; on attribuait autrefois des propriétés particulières au coeur et au sang de l'animal. Le principal marché est celui de Bab Jebli, à Sfax. Les carapaces sont habituellement jetées, et l'on en voit très peu qui soient mises en vente pour les touristes. Des documents du début du vingtième siècle et de la fin du dix-neuvième siècle parlent aussi de la consommation de tortues, qui semble traditionnelle; on capturait jadis les animaux à l'occasion de la pêche, mais on les prenait parfois au harpon ou on les ramassait lorsqu'elles se reposaient à la surface (Laurent et coll., sous presse).

Une récolte de cette importance a probablement des conséquences sérieuses pour les populations de tortues méditerranéennes, et il convient d'examiner la possibilité de mettre fin à cette exploitation, ou au moins de la réduire.

On dit que Dermochelys sert également à l'alimentation, en plus des usages médicinaux (Baccar, in litt.); cette espèce est très rarement utilisée à de telles fins ailleurs, de sorte qu'il serait intéressant de rassembler davantage de données sur l'emploi qu'on en fait en Tunisie.

\subsubsection{Mesures de protection}

Il $\mathrm{n}^{\prime} \mathrm{y}$ a pas de législation nationale de protection en vigueur, bien que, selon Laurent et coll. (sous presse), le Commmissariat général à la pêche ait publié la note n 1155 du 10 juin 1987 priant les autorités régionales d'interdire la capture de tortues, comme le demande la Convention africaine de 1977, dont la Tunisie est signataire. 


\subsection{TURQUIE}

\subsubsection{Observations générales}

Selon l'information disponible (d'ailleurs incomplète pour une grande partie de l'Afrique du nord), la Turquie serait de loin le pays le plus important de la Méditerranée pour le nombre total de femelles reproductrices par saison, ainsi que pour la diversité des espèces (nidificat ion à

la fois de Caretta et de Chelonia). Le développement touristique touche une large part du littoral méditerranéen de la Turquie, et l'on sait que des projets de nouveaux développements existent un peu partout, le long des côtes.

Il y a des nidifications presque partout entre Dalyan, à l'ouest, et la frontière syrienne, à l'est, lorsqu'il existe des plages appropriées et non trop perturbées (Baran et Kasparek, 1989a). La longueur totale des plages réparties le long des côtes égéennes et méditerranéennes de la Turquie est d'environ $600 \mathrm{~km}$. La plupart des nidifications importants ont lieu sur 13 plages principales, s'étendant au total sur $120 \mathrm{~km}$; des nidifications plus modestes ont lieu sur quatre autres plages, d'une longueur totale de $19 \mathrm{~km}$ (Baran et Kasparek, 1989a).

Un travail de terrain effectué par Geldiay et ses collaborateurs jusqu'en 1979 couvrait cinq lieux de ponte dans les secteurs occidentaux et centraux entre Dalyan et Alanya, sur un espace total de près d'une centaine de kilomètres, et une activité subséquente, de 1980 à 1982, intéressait une bonne partie de la côte orientale, entre Silikfe, Mersin et Yumurtalik (Geldiay et coll., 1982; Geldiay, 1984). Les méthodes de recherche employées n'ont pas été précisées.

Le projet lancé en 1988 par le Fonds mondial pour la nature, la CEE et le DHKD a permis la première large inspection des lieux de nidification de tortues de la côte méditerranéenne de Turquie; il avait pour but principal de reconnaître les plages de ponte et d'en établir une appréciation comparée (Baran et Kasparek, 1989a). On a relevé les traces de femelles émergentes en trois occasions au cours des trois mois de travail sur le terrain. Il importe de noter que les chiffres publiés ne prétendent pas représenter que des empreintes fraiches (Kasparek, in litt., 8 décembre 1988), et qu'ils comprennent généralement des empreintes datant d'une période inconnue, mais toujours visibles sur la plage. Aussi est-il rarement possible d'estimer le nombre de nids aménagés par nuit, et impossible de faire une estimation fiable du nombre des nidifications par saison. Dans la mesure où des empreintes antérieures peuvent être effacées d'une inspection à l'autre, les chiffres sous-estiment le nombre de nidifications réel. Les comptages ont été plus fréquents sur deux des principales plages de Chelonia (Baran et Kasparek, 1989a; Langeveld et Sarigül, 1988), et permettent une estimation de la quantité de nids.

On a constaté des nidifications de Caretta en plusieurs endroits, depuis la région de Dalyan, vers I'est, au moins jusqu'à la plage de Tuzla (entre Kazanli et Akyatan, dans le delta de Cukurova, lequel est surtout fréquenté par Chelonia), et l'on trouve C. mydas plus rarement, depuis la région de Manavgat-Kizilot, dans la baie d'Antalya, et vers l'est jusqu'à Samandagi, près de la frontière syrienne (Baran et Kasparek, 1989a; Geldiay et coll., 1982; Geldiay, 1984). Les nidifications importants de Chelonia sont surtout restreints à Kazanli et Akyatan, dans le delta de Cukurova, au sud d'Adana. On 
ne sait exactement dans quelle mesure les plages orientales de Chelonia sont également fréquentées par Caretta; mais il semble (si l'on en croit Geldiay, 1984; Langeveld et Sarigül, 1988) que presque tous les nids sont de Chelonia.

\subsubsection{Plages de ponte et nombres: Caretta caretta}

Geldiay a repéré des nidifications à Dalyan, Fethiye, Kas, Finike, Kumluca, Kemer, Antalya, Belek, Side, Alanya, Gazipasa, Silifke et Mersin (Geldiay et coll., 1982; Geldiay, 1984). Il peut y avoir eu, et il y a peut-être encore, des nidifications sur la côte égéenne, et il y en a certainement en plusieurs endroits de la côte méditerranéenne non examinés par Geldiay, mais étudiés par Baran et Kasparek (1989a).

Les sites dont il est question ci-dessous sont ceux que Baran et Kasparek (1989a) tiennent pour les plus importantes plages de ponte de Turquie.

\subsubsection{Dalyan}

Les renseignements sur les émergences et les nidifications de tortues à Dalyan, recueillis au cours de la saison 1987 , sont dans l'ensemble compatibles avec les données présentées par Geldiay. L'information récente nous vient de Riccardo Jesu (étudiant chercheur de Gênes) et d'une équipe organisée par le Groupe Kavala. Jesu a parcouru la plage chaque nuit, pendant un temps variable, puis le matin suivant, aux fins de confirmation, et il a enregistré les nidifications et les émergences sans nidification. Son enquête, commencée en juin 1987, ne couvrait pas la première partie de la saison de ponte. Les données fournies par Jesu sont présentées au tableau 6 .

Si la moyenne de 4,8 nids par nuit, pour la période de 32 jours enregistrée par Jesu, s'est maintenue pendant les 60 jours de la saison principale de nidification de juin et juillet, quelque 288 nids auraient été aménagés, et peut-être 350 au total en 1987 (en comptant des nidifications épars au commencement et à la fin de la saison, c'est-à-dire au début de mai et au mois d'août). Nous sommes ainsi très près du chiffre de 330 nids par saison publié par Geldiay et coll. (1982). Un total saisonnier de 350 nids correspondrait à environ 77 nids par kilomètre (pour une longueur de plage de 4,5 kilomètres), et à 117-140 femelles reproductrices (si l'on suppose une moyenne de ponte par saison de 3 ou 2,5 respectivement).

D'autre part, le chiffre de 4,8 nids par jour, en saison, rapporté par Jesu, ne confirme pas l'estimation, rapportée par Geldiay (1984), de plus de 4 nids par jour pour chaque kilometre de plage. Les données de 1987 ne suggèrent qu'une moyenne d'environ 1,1 nid par jour et par kilomètre. Cette disparité n'indique pas nécessairement une diminution du nidification des tortues à Dalyan: le nombre de nidifications est souvent variable d'une saison à l'autre, à travers le monde, et de très grandes différences ne sont pas rares. Mais il semble certain que l'activité humaine, sur la plage de Dalyan, a été excessive au cours de la saison 1987. Non seulement de larges échos dans les média, sur le plan national et international, ont amené des nombres variables de touristes à visiter la plage la nuit pour y voir des tortues, mais plusieurs groupes de chercheurs (de divers niveaux de compétence) ont procédé à des enquêtes intéressant la région de Dalyan et les tortues reproductrices. On pense que, dans ces conditions, un assez grand nombre de femelles ont pu être dissuadées de nicher à Dalyan en 1987. On dit que les perturbations se sont encore accrues en 1988 , en raison à la fois du tourisme et de certains chasseurs de tortues. 
Tableau 6. Données sur l'émergence et le nidification des tortues sur la plage de Dalyan, du 4 juin au 5 juillet 1987, période qui représente environ la moitié de la saison principale de ponte. Informations recueillies par Riccardo Jesu (Gênes) aux fins d'une maitrise es-sciences. $L^{\prime}$ auteur remercie vivement $\mathrm{R}$. Jesu d'avoir bien voulu mettre ces données à sa disposition.

$\begin{array}{lll}\text { Date } & \begin{array}{l}\text { Total des } \\ \text { émergences }\end{array} & \begin{array}{l}\text { Emergence } \\ \text { seulement } \\ \text { (sans nid) }\end{array} \\ & & \text { Nids }\end{array}$

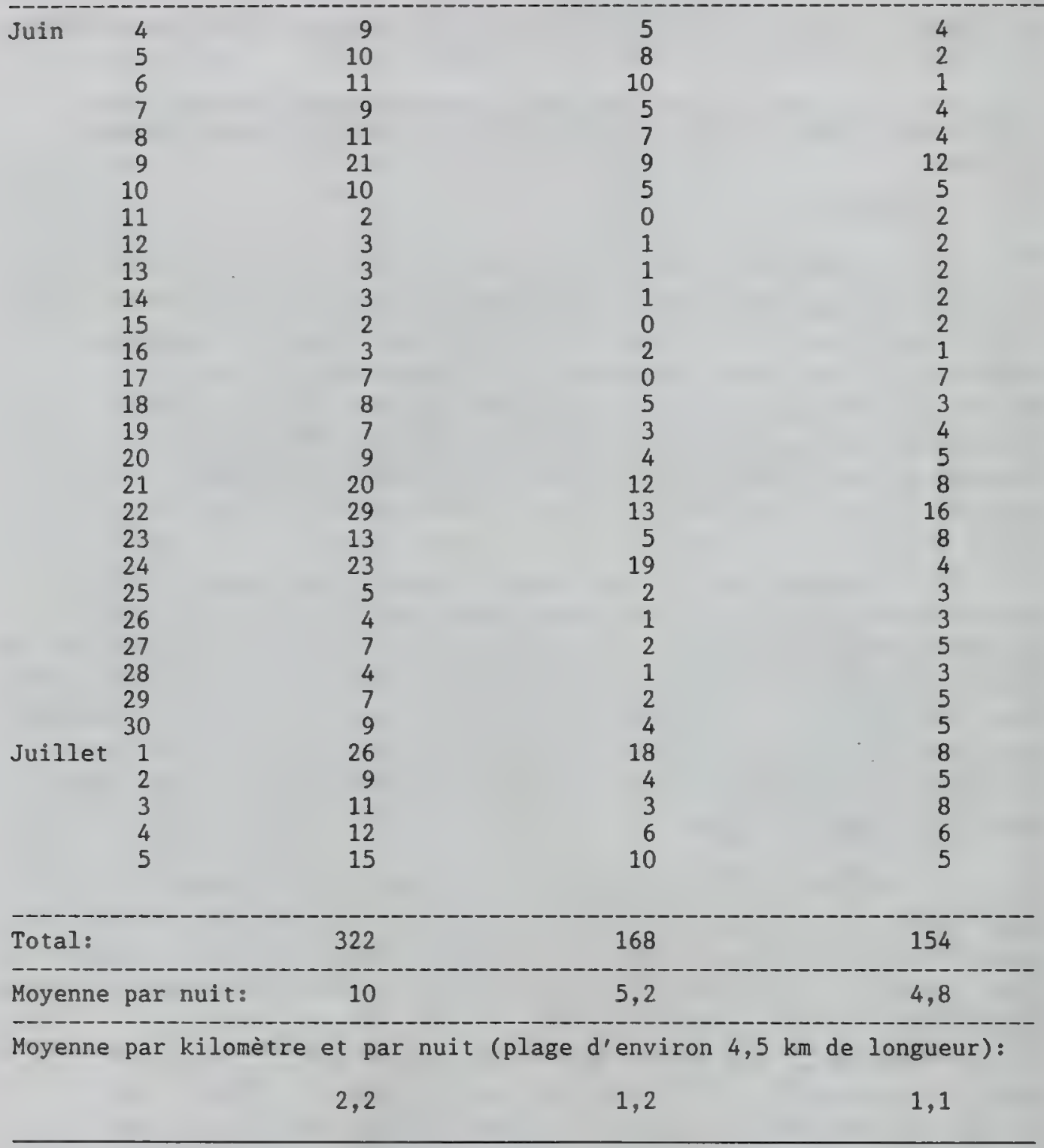


Tableau 7. nidifications de Caretta à Dalyan, 1987-1989. Voir clef ci-dessous.

\begin{tabular}{lrrr}
\hline Année & 1987 & 1988 & 1989 \\
\hline Nids & $\begin{array}{r}154^{\mathrm{a}} \\
\text { c. } 300^{\mathrm{b}}\end{array}$ & $145^{\mathrm{c}}$ & $250^{\mathrm{c}}$ \\
\hline
\end{tabular}

Glef: ${ }^{a}=$ données recueillies du 4 juin au 5 juillet par $\mathrm{R}$. Jesu; cestimé du total 1987 extrapolé des données de Jesu (voir texte); $c^{c}=$ données recueillies par l'Université de Hacettepe, citées par Sarigül (1989).

Sarigül (1989) signale que l'Université de Hacettepe a compté 145 nids en 1988 , et 250 en 1989 , ce qui équivaut à 56 par kilomètre pour la saison.

Le secteur de Dalyan-Iztuzu a été déclaré zone spécialement protégée fin 1988 (le lac adjacent de Köycegiz lui a été ensuite adjoint). La DHKD (Societé pour la protection de la nature) et l'AGA (Aktions Gemeinschaft Artenschutz) ont réalisé en 1989 un programme de protection des plages couvrant la gestion de l'accès et l'éducation du public.

\subsubsection{Sarigerme-Dalaman}

La plage, de 10 à 11 kilomètres de longueur, est située à environ dix kilomètres (en ligne droite) à l'est de celle de Dalyan. Des observations préliminaires effectuées en juillet 1987 (Groombridge, inédit) dans le secteur le plus occidental de la plage, près de Sarigerme, $n^{\prime}$ ont révélé aucune empreinte, bien que des pêcheurs locaux aient signalé que des femelles reproductrices et des nouveau-nés avaient été vus régulièrement depuis sept ans, et que des tortues sont souvent capturées accidentellement dans le secteur.

Celui-ci est fortement perturbé par un grand camp touristique, et les nidifications, s'il en est, sont probablement très rares. Vers l'est, on a trouvé deux nids, le 12 juillet, sur une distance d'un kilomètre, le long de l'aéroport, et à l'ouest de l'embouchure de l'Acisu (dans un secteur généralement trop rocheux pour le nidification des tortues), et cinq nids (dont trois ouverts par des prédteurs) sur un kilomètre de plage à l'est de l'embouchure de l'Acisu (Groombridge, inédit). Des informateurs locaux ont signalé quelques nidifications de tortues marines, mais surtout vers l'extrémité orientale de la plage de Dalaman.

La plage centrale, à l'ouest de l'aéroport de Dalaman et vis-à-vis la ferme d'Etat, a été examinée, début juin 1987, par Kinzelbach et Whitmore, qui y ont dénombré quelque 19 ensembles $\mathrm{d}$ 'empreintes récentes (G. Whitmore, comm. pers.). Des informateurs locaux ont confirmé que des tortues nichaient en ces lieux, mais que les nids étaient très fortement pillés par les renards. L'enquête de 1988 a confirmé l'importance particulière de ce secteur, qui s'étend sur 3,8 km, entre l'embouchure du Dalaman et le déversement de la papéterie de SEKA; le secteur adjacent, de $1,9 \mathrm{~km}$, jusqu'à l'embouchure de l'Acisu, constitue également une plage de ponte (Baran et Kasparek, 1989a). Les comptages d'empreintes et de nids trouvés chez Baran et Kasparak (1989a) sont présentés au tableau 8; ils permettent une comparaison entre divers secteurs de la plage de Sarigerme-Dalaman, mais non une estimation du nombre de nids par saison (voir section 3.18.1). 
Selon les projets actuels d'investissement, la plus grande partie de la plage de Sarigerme-Dalaman, sauf un secteur d'1,5 km le long de l'aéroport, est promise au développement touristique, notamment à la construction d'hôtels (Baran et Kasparek, 1989a). On édifiait en 1987 un vaste complexe hôtelier sur un éperon rocheux dominant le secteur occidental, et un autre complexe dominant la partie orientale. Baran et Kasparek (1989a) recommandent de limiter toute construction future au secteur de Sarigerme, et de protéger strictement les plages plus centrales où se manifeste la plus grande activité de nidification. Ils soulignent également la nécessité de limiter les effluents de la papéterie de SEKA.

Tableau 8. Résumé des données de nidification réunies lors de l'examen de la plage de Sarigerme-Dalaman en 1988 (Baran et Kasparek, 1989a). Dans ce tableau et dans les suivants, les chiffres de la colonne "émergences" comprennent le nidification et les empreintes sans nidification.

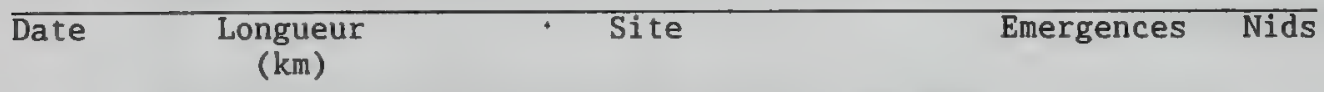

24 juin
30 juin
11 juillet
12 juillet
12 août

3,8 Fleuve Dalaman-déversement SEKA 1,9 Déversement SEKA-embouchure Acisu Fleuve Dalaman-Déversement SEKA Déversement SEKA-embouchure Acisu Déversement SEKA-embouchure Acisu Fleuve Dalaman-Déversement SEKA Fleuve Dalaman-Déversement SEKA Déversement SEKA-embouchure ACISU

$\begin{array}{rr}24 & 13 \\ 5 & 4 \\ 28 & 20 \\ 20 & 11 \\ 11 & 2 \\ 37 & 24 \\ 73 & 17 \\ 4 & 3\end{array}$

Comptage du Dalaman - déversement SEKA pendant la période: 162

En pourcentage du comptage total Sarigerme-Dalaman:

\subsubsection{Fethiye-Calis}

Le secteur septentrional de la plage, long de 4,8 km, proche de Calis, et qui commence à quelque $5 \mathrm{~km}$ au nord de Fethiye, est classé, par Baran et Dasparek (1989a), comme une importante plage de ponte de Caretta. Il s'agit d'une plage d'environ $50 \mathrm{~m}$ de largeur, généralement en mosaïque galets-sable. Geldiay (1984) a rangé ce secteur parmi les sites de nidification moyennement denses, avec un à quatre nids par nuit et par kilomètre. Le comptage des empreintes et des nidifications extrait de Baran et Kasparek (1989a) est présenté au tableau 9.

La plage fait l'objet d'une forte exploitation touristique, notamment au voisinage de camps de vacances, à l'extrémité septentrionale du secteur central, tandis qu' on extrait du gravier dans le secteur nord (Baran et Kasparek, 1989a).

Le site a été classé en 1988 comme "zone spécialement protégée". 
Tableau 9. Résumé de données de nidification réunies sur la plage nord de Fethiye-Calis lors de l'enquête 1988 (Baran et Kasparek, 1989a).

\begin{tabular}{lclrc}
\hline Date & $\begin{array}{c}\text { Longueur } \\
(\mathrm{km})\end{array}$ & \multicolumn{1}{c}{ Sites } & Emergences & Nids \\
\hline \multirow{2}{*}{25 juin } & 1,0 & Secteur nord & 3 & 3 \\
& 1,5 & Secteur central & 23 & $19 ?$ \\
13 & & & 19 & $18 ?$ \\
& 2,3 & Secteur sud, jusqu'à Calistepe & 0 & 0 \\
& & Secteur nord & 52 & 50 \\
& & Secteur central & 11 & 10
\end{tabular}

Comptage total dans le secteur central au cours de la période:

\subsubsection{Patara}

Cette plage de sable de 11.8 kilomètres de longueur est à quelque $80 \mathrm{~km}$ à vol d'oiseau à l'est de Dalyan, au sud de Kumluova et de Xanthos, et elle est partagée en deux parties par l'embouchure du fleuve Esencay. La moitié orientale, entre l'Esencay et Patara, est relativement haute et sablonneuse, présentant une pente marquée au-dessus de l'estran. Selon Baran et Kasparek (1989a), c'est la plage que Geldiay (1984) désigne sous le nom de "Kas", et qu'il décrit comme d'importance moyenne, avec 1 à 4 nids par jour et par kilomètre. Des observations imprévues de l'extrémité orientale de la plage proche de Gelemis, le 8 juillet 1987, ont révélé quatre nouveaux nids de Caretta et deux aller-et-retour ont été relevés sur le kilomètre et demi parcouru à pied le 8 juillet 1987 (Groombridge, observations personnelles); ce seul secteur semble avoir compté autant de nids nouveaux que les 4,5 kilomètres de la plage de Dalyan en comptaien: chaque nuit, du 5 au 12 juillet. L'examen à la jumelle d'une partie des trois à quatre kilomètres restants vers l'ouest, jusqu'au fleuve, révèlait nombre d'empreintes d'émergences apparemment récentes, allant jusqu'à la laisse de haute mer. Une bonne fraction de la partie occidentale de la plage est de sable assez limoneux et compact, souvent bas et très plat. Une empreinte ancienne, qui n'a mené à aucun nid, était probablement le fait de $C$. mydas; l'un de deux nids de caouanne avait été ouvert et des mouettes avaient récemment mangé les oeufs mis au jour. Ces derniers se trouvaient au-dessous du niveau de la mer. Une bonne partie de la plage était l'objet d'érosion éolienne et, bien qu'on ait trouvé une dizaine de concentrations de coquilles vides d'oeufs de tortue - probablement révélatrices de nidification aucune autre empreinte n'était visible.

Gramentz (in litt., 13 août 1988) constata que l'activité touristique s'étaít énormément accrue pendant la saison 1988 , en même temps que les logements disponibles (on comptait une douzaine de pensions contre deux seulement en 1987); et il y avait même des installations sur la plage (et un projet d'asphaltage du sentier menant à celle-ci). Cet auteur a également reçu un rapport fiable sur la collecte des oeufs. Barton (comm. pers., octobre 1988) signale également une exploitation touristique intense de la plage de Patara, avec des tracteurs descendant et remontant la pente du rivage pour approvisionner les buvettes en rafraîchissements. Il se fait actuellement un grand développement touristique aux environs de Gelemis. 
Bien qu'empêché de poursuivre par des exercices militaires qui eurent lieu sur la plage à partir du 10 juillet, Dieter Gramentz put examiner l'activité de nidification à l'est de l'Esencay, entre le 7 juin et le 9 juillet, et il nous a aimablement communiqué ses résultats (Gramentz, in litt.). Pendant 32 nuits, il enregistra 124 émergences, toutes de Caretta, et au moins 14 nidifications, la plupart immédiatement à l'est de l'Esencay. Il bagua huit femelles. Le tableau 10 présente les comptages d'empreintes et de nids extraits de Baran et Kasparek (1989a), qui soulignent la proportion extrêmement élevée d'émergences sans nidification. Lors d'un bref examen, en juillet 1987, on a constaté que presque toutes les empreintes se trouvaient sur la moitié orientale de la plage (Groombridge, obs. pers.), tandis qu'à la saison 1988, 60 \% des empreintes étaient sur la moitié occidentale (Baran et Kasparek, 1989a). C'est peut-être la conséquence d'un accroissement des perturbations coté est.

Patara a été déclaré zone spécialement protégée le 2 mars 1990, comme en fait foi le Journal officiel n 20449 (Whitmore et coll., sous presse, 1990).

Tableau 10. Résumé de données de nidification réunies sur la plage de Patara lors d'une enquête de 1988 (Baran et Kasparek, 1989a).

Date Longueur $(\mathrm{km}) \quad$ Site Emergences Nids

$\begin{array}{lll}10 \text { juin } & 6.0 & \begin{array}{l}\text { Secteur oriental } \\ \text { Secteur occidental }\end{array} \\ 26-28 \text { juin } & 4.9 & \begin{array}{l}\text { Secteur occidental } \\ 13 \text { juillet }\end{array} \\ & & \begin{array}{l}\text { Secteur oriental } \\ \text { Secteur occidental }\end{array} \\ 14 \text { août } & & \text { Secteur oriental } \\ & & \text { Secteur occidental }\end{array}$

Comptage total au cours de la période:

$\begin{array}{rc}17 & 2 ? \\ 12 & 1 \\ 26 & 9 ? \\ 10 & 1 \\ 38 & >7 \\ 8 & 0 ? \\ 3 & 2 \\ . & .\end{array}$

\subsubsection{Finike-Kumluca}

Cette plage s'étend sur quelque $20,5 \mathrm{~km}$ autour de la baie de Finike. Geldiay (1984) suggérait une moyenne de plus de quatre nidifications de Caretta par jour et par kilomètre le long du secteur occidental de la plage, près de Finike, et de un à quatre nidifications de Caretta par jour et par kilomètre vers le secteur oriental, plus près de Kumluca et de Mavikent. Baran et Kasparek (1989a) considèrent le secteur oriental comme l'une des plus importantes plages de ponte de Caretta sur les côtes turques. Cette partie est surtout garnie de galets, alors que le sable prédomine à l'ouest. Les cabanons dont se servent les gens du voisinage pendant les mois d'été constituent la principale influence humaine sur ces plages; ils sont surtout concentrés dans le secteur oriental, mais, dans leur ensemble, intéressent l'équivalent de la moitié de la longueur de plage totale (Baran et Kasparek, 1989a). La plupart des gens arrivent en juillet, avec objets de ménage, voitures et animaux domestiques; les cabanons sont desservis par une ligne électrique de Mavikent. Comme ils sont 
inoccupés pendant une bonne partie de la période de ponte, le nidification lui-même est probablement peu perturbé; on croit cependant que les nouveau-nés sont sérieusement atteints par la pollution lumineuse, le passage de véhicules à moteur et la prédation par les animaux domestiques (Baran et Kasparek, 1989a). Le ministère du Tourisme projette un développement du secteur oriental.

Le comptage des empreintes et des nids effectué par Baran et Kasparek (1989a) est présenté au tableau 11. La plupart des nids se trouvaient dans le secteur oriental, au voisinage de Mevikent, et surtout dans les parties les plus centrales de ce secteur. En une occasion, on a procédé deux jours de suite ( 27 et 28 juin) à des comptages sur la grande plage centrale et orientale. Les chiffres du 28 juin - 21 empreintes et 14 nids - représentent probablement des émergences nouvelles de la nuit du 27 au 28 juin. Selon sette hypothèse, la densité était relativement forte: 5,6 nids par kilomètre et par nuit.

Tableau 11. Résumé de données de nidification réunies sur la plage de Finike-Kumluca lors de l'examen de 1988 (Baran et Kasparek, 1989a).

Date

$\underset{(\mathrm{km})}{\text { Longueur }}$
Site

Emergences Nids

$\begin{array}{lr}13 \text { juin } & 4.7 \\ & 6.2 \\ 27 \text { juin } & 9.4 \\ & 11.5 \\ 28 \text { juin } & 7.4 \\ & 2.3\end{array}$

16 juillet 11.5

15 août $\quad 4.7$

16 août $\quad 4.8$

17 août $\quad 4.7$

17 août $\quad 6.6$

19 août 3.7

\author{
Secteur occidental (partie) \\ Secteur oriental (partie) \\ Secteur occidental \\ Secteur oriental \\ Secteur oriental (partie) \\ Secteur méridional, \\ jusqu'à Calistepe \\ Secteur oriental \\ Secteur occidental (partie) \\ Secteur oriental (partie) \\ Secteur occidental (partie) \\ Secteur oriental (partie) \\ Secteur oriental (partie)
}

\begin{tabular}{rc}
14 & 7 \\
32 & 6 \\
13 & 8 \\
35 & 30 \\
40 & 25 \\
& \\
19 & $18 ?$ \\
181 & 71 \\
0 & 0 \\
102 & 0 \\
0 & 0 \\
8 & 1 \\
0 & 0 \\
\hline
\end{tabular}

Comptage total pour le secteur oriental au cours de la période:

\subsubsection{Région de Belek}

La partie de côte qui sépare Antalya et Side, soit une trentaine de kilomètres, comprend plusieurs plages où l'on a constaté des nidifications de tortues; Geldiay (1984) traite de trois sites (Antalya, Belek-Serik, Side) séparément, tandis que Baran et Kasparek suggèrent qu'il faut traiter toute cette côte comme unité. Selon Geldiay (1984), Artalya et Belek/Serik accueillent plus de quatre nidifications par kilomètre et par jour, tandis que side reçoit de un à quatre reproductrices. Les données présentées par Baran et Kasparek (1989a) suggèrent qu'il y a des nidifications plutôt isolés, en plusieurs lieux, le long de cette côte; on trouvera des chiffres choisis au tableau 12. 
Sur les $13,3 \mathrm{~km}$ de la plage de Belek-Cakallik, le 18 juillet, on a estimé que 45 nids ( 71 émergences) dataient de un ou deux jours, ce qui équivalait à quelque trois nids par kilomètre et par jour. Selon Baran et Kasparek, Geldiay appelle Belek la plage de Belek-Cakallik, et Serik la plage de Belek-Köprü Cayi. La plus grande partie de cette côte est atteinte par les activités touristiques locales et internationales, les véhicules de plage, les prédateurs de nids, et I'on projette d'autres développements.

Tableau 12. Résumé de données choisies de nidification réunis sur la côte de Belek entre Antalya et Side lors de 1'enquête de 1988 (Baran et Kasparek, 1989a).

\section{Date}

Longueur $(\mathrm{km})$

Site

Emergences Nids

$\begin{array}{ll}17 \text { juin } & 10,8 \\ 18 \text { juillet } & \\ 18 \text { juin } & 13,3 \\ 25 \text { juin } & \\ 18 \text { juillet } & \\ 24 \text { août } & \\ 18 \text { juin } & 8,5 \\ 19 \text { juillet } & \\ 21 \text { juin } & 6,6 \\ 19 \text { juillet } & \\ 26 \text { août } & \end{array}$

\subsubsection{Région de Kizilot}

Baran et Kasparek (1989a) notent que le secteur occidental (plage orientale de Side) connait des concentrations de nidifications de Caretta exceptionnelles en Turquie; il y a également des nidifications importants de Caretta, et plus rarement de Chelonia, dans le secteur oriental (Kizilot). Le développement touristique envahit une bonne partie de la plage de Side, et la plupart des nidifications sont repérés dans la portion orientale la moins aménagée, où $l^{\prime}$ on en trouve parfois entre les cabanons qu'utilisent les habitants en été, et parfois sur la plage adjacente non perturbée qui s'étend à l'est jusqu'à l'embouchure du fleuve Manavgat. La plage occidentale de Kizilot s'étend du Manavgat jusqu'à l'Hôtel Cactus Beach, à $1 \mathrm{~km}$ à l'est de Kizilot. On a enregistré 75 nids le 24 juillet et, si l'on suppose qu'aucun $n^{\prime}$ a été compté deux fois, on peut penser que tous ces nids ont été aménagés au cours des trois nuits écoulées depuis le comptage précédent du 21 juillet. Il y aurait donc eu 25 nids par nuit, sur $7,7 \mathrm{~km}$, soit 3,2 nids par $\mathrm{km}$ et par nuit. Ce secteur fait l'objet d'extraction de sable et de galets, d'une activité locale et internationale de vacances, et deux grands hôtels étaient en construction en 1988. Il y a aussi des nidifications importants sur la plage orientale de Kizilot, qui est le théâtre d'aménagements hôteliers et récréatifs.

Baran et Kasparek (1989a) recommandent fortement que des parties des plages de l'est de Side, de l'ouest et de l'est de Kizilot soient gérées comme des réserves strictes de nidification. 
Tableau 13. Résumé de données choisies de nidification réunies dans la région de Kizilot lors de l'enquête de 1988 (Baran et Kasparek, 1989a)

\begin{tabular}{|c|c|c|c|c|}
\hline Date & Longueur ( $\mathrm{km})$ & Site & Emergences & Nids \\
\hline $\begin{array}{l}21 \text { juin } \\
24 \text { juillet } \\
22 \text { juin } \\
21 \text { juillet } \\
24 \text { juillet } \\
23 \text { juin } \\
24 \text { juillet }\end{array}$ & $\begin{array}{l}3,5 \\
7,7\end{array}$ & $\begin{array}{l}\text { Plage de Side est (partie) } \\
\text { Plage de Side est (partie) } \\
\text { Plage de Kizilot ouest } \\
\text { Plage de Kizilot ouest } \\
\text { Plage de Kizilot ouest } \\
\text { Plage de Kizilot est } \\
\text { Plage de Kizilot est }\end{array}$ & $\begin{array}{r}20 \\
11 \\
54 \\
23 \\
143 \\
20 \\
75\end{array}$ & $\begin{array}{r}10 \\
6 \\
38 \\
16 \\
75 \\
13 \\
47\end{array}$ \\
\hline
\end{tabular}




\subsubsection{Demirtas}

Cette plage de $7,4 \mathrm{~km}$ est située près de la petite ville de Demirtas. Il y a un hôtel, une activité de vacances modeste et quelque extraction de sable et de galets. Le ministère du Tourisme ne projette pas de nouveau développement.

Tableau 14. Résumé de données de nidification recueillies sur la plage de Demirtas lors de l'enquête de 1988 (Baran et Kasparek, 1989a)

Date Longueur $(\mathrm{km})$ Site Emergences Nids

$\begin{array}{llllr}15 \text { juin } & 7,4 & \text { Demirtas } & 11 & 3 \\ 26 \text { juillet } & & \text { Demirtas } & 74 & 38 \\ 15 \text { août } & & \text { Demirtas } & 16 & 3\end{array}$

\subsubsection{Gazipasa Ciftlik}

Environ 1,6 km sur les 2,4 de cette plage principalement garnie de galets convient au nidification. Il n'y a pas d'activité touristique, mais la portion méridionale fait l'objet d'extraction de sable et de galets. Il y aurait eu dans le passé ramassage des oeufs, et il semble que $I^{\prime}$ on tue parfois des tortues pour employer la carapace à des fins décoratives. Baran et Kasparek (1989a) estiment la densité de nidifications - principalement ou entièrement de Caretta relativement forte.

Tableau 15. Résumé de données de nidification recueillies sur la plage de Gazipasa Ciftlik lors de l'enquête 1988 (Baran et Kasparek, 1989a)

Date

Longueur $(\mathrm{km}) \quad$ Site

Emergences Nids

\begin{tabular}{lrlrr}
\hline 16 juin & 1,6 & Gazipasa Ciftlik & 8 & 3 \\
26 juillet & & Gazipasa Ciftlik & 39 & 28 \\
\hline
\end{tabular}




\subsubsection{Delta de Göksü}

Cette grande région limnétique est bordée de plages totalisant quelque 22,4 km de longueur; la plupart des nidifications de tortues - principalement ou entièrement de Caretta - ont lieu sur une bande d'environ $5 \mathrm{~km}$, à mi-distance des $1 \overline{0,5 \mathrm{~km}}$ du rivage occidental. La grande ville la plus proche est Silifke, et ce secteur pourrait correspondre en partie aux plages de Silifke dont parle Geldiay (1984). Les 2 à $3 \mathrm{~km}$ de la portion nord de la côte occidentale connait une activité touristique, avec plusieurs villages de vacances pour la population turque; les $3 \mathrm{~km}$ de la portion sud sont très plats et souvent délavés par la mer. La portion centrale utilisée par les tortues fait parfois l'objet d'extraction de sable. Baran et Kasparek suggèrent que $36 \%$ (30 unités) des 84 empreintes observées le 12 juillet dataient probablement de la nuit précédente; en calculant avec le même pourcentage, on pourrait penser qu'environ 8 nids avaient été aménagés, soit une densité de 0,8 nid par $\mathrm{km}$ et par nuit. La plupart des émergences $n^{\prime}$ ont pas abouti à un nidification.

Le delta de Göksü a été déclaré zone spécialement protégée le 2 mars 1990, comme le notait le Journal officiel n² 20449 (Whitmore et coll, sous presse, 1990).

Tableau 16. Résumé de données de nidification recueillies au delta de Göksũ lors de I'enquête de 1988 (Baran et Kasparek (1989a)

Date Longueur $(\mathrm{km})$ Site Emergences Nids

$\begin{array}{lllll}21 \text { juin } & 10,5 & \text { Delta de Göksü } & 39 & 17 \\ 12 \text { juillet } & & \text { Delta de Göksü } & 84 & 22 \star\end{array}$

* Calculé d'après des chiffres de Baran et Kasparek (1989a:99).

\subsubsection{Plages de ponte de caouannes: résumé}

Si l'on ne considère que Caretta, pour laquelle Geldiay fournit des estimations du nombre de nids par kilomètre et par saison (Dalyan, Kumluca, Side, Alanya), Dalyan est de loin la plage la plus importante. La densité de quelque 70 nids par kilomètre pour la saison (en supposant une plage de $4,5 \mathrm{~km}$ de longueur, et non de sept comme le dit Geldiay) est plus du double de celle d'une trentaine de nids constatée aux deux sites les plus comparables. Dans son document de 1984, Geldiay (figure 9) indiquait que trois autres sites qu'il avait examinés (Finike, Antalya et Serik) avaient une densité de nids journalière comparable, toujours supérieure à quatre nids par jour pour la saison. La longueur de plage de ponte disponible et le total de nids par saison sur ces autres sites ne sont pas indiqués, de sorte que l'on ne peut calculer le nombre de nids par kilomètre et par saison. 
Des données inédites réunies en 1987, et une information obtenue grâce à l'enquête menée en 1988 par le Fonds mondial pour la nature (WWF), la CEE et le DHKD (Baran et Kasparek, 1989a) indiquent que plusieurs sites, outre Dalyan, accueillent des nombres importants de nidifications de Caretta. On n'a suffisamment inspecté aucun des autres sites au cours de la saison de ponte, de sorte qu'on ne possède pour aucun de données comparables à celles de Dalyan, d'abord examinée par Jesu et par l'équipe de Kavala en 1987. Les renseignements incomplets dont nous disposons suggèrent que certaines des neuf autres plages classées comme sites de nidification majeurs par Baran et Kasparek (1989a) pourraient avoir accueilli en 1988 un nombre de nids comparable à celui de Dalyan en 1987, bien que la densité de nidification soit inférieure.

Des données préliminaires tabulées dans un avant-rapport de projet (Baran et Kasparek, 1988) indiquent qu'on a relevé quelque 630 nids de Caretta entre Dalyan et le delta de Göksü, au cours de la période du 29 mai au 18 juillet 1988; c'est-à-dire une moyenne d'environ 13 nids par nuit, pendant 50 jours. La majorité des nids se répartissait sur une dizaine de plages de ponte principales. Comme l'on n'avait pas couvert la première partie de la saison de nidification, et qu'on avait pu manquer de nombreux nids entre les visites, ces chiffres pourraient représenter une sous-estimation sensible. Les données en question ne sont pas tabulées dans le rapport définitif (Baran et Kasparek, 1989a), mais sont compatibles avec les chiffres donnés dans l'examen de plages de ponte particulières. Une extrapolation de ces chiffres pour refléter une saison de nidification d'environ 110 jours (de la mi-mai à la fin août) suggère un minimum de 1.650 nids pour la saison 1988; en supposant trois nids par femelle, on peut penser qu'un minimum de 550 femelles ont niché. Les données de Geldiay et coll. (1982) et Geldiay (1984) suggèrent, sur la base d'une hypothèse semblable, qu'environ 1000 Caretta ont niché par saison. Il semble juste de supposer que le contingent moyen de reproductrices par saison, en Turquie, devrait se situer entre les deux extrêmes de 550 et 1000 femelles.

\subsubsection{Lieux et nombres de nidifications: Chelonia mydas}

On relève des nidifications de Chelonia mydas le long des parties centrale et orientale de la baie d'Antalya (Geldiay et coll., 1982: 429, fig. 3), notamment sur la plage de Belek (longue de $40 \mathrm{~km}$, à $\left.36^{\circ} 50^{\prime} \mathrm{N}, 30^{\circ} 58^{\prime} \mathrm{E}\right)$, celle de Side $\left(30 \mathrm{~km}\right.$, à $36^{\circ} 51^{\prime} \mathrm{N}$, et $31^{\circ} 28^{\prime} \mathrm{E}$ ) et celle d'Alanya $\left(12 \mathrm{~km}\right.$, à $\left.36^{\circ} 36^{\prime} \mathrm{N}, 32^{\circ} 05^{\prime} \mathrm{E}\right)$. On $\mathrm{n}^{\prime}$ a pas trouvé de nids de $\mathrm{C}$. mydas à l'ouest de Belek, et Alanya est la plage de ponte la plus orientale à être signalée. Les études ultérieures, de 1980-1982 (Geldiay, 1984: 72, fig. 9), ne semblent avoir révélé aucun nid de C. mydas à Belek ou à Side; on mentionne des nidifications près d'Alanya, mais de plus importants à Mersin, Tuzla, Karatas et Yumurtalik.

L'étude "WWF-CEE-DHKD" (1988) a révélé que les nidifications importants de Chelonia se restreignaient surtout à Kazanli et Akyatan, dans le delta de Cukurova, au sud d'Adana, avec certains nids à Samandagi, près de la frontière syrienne (Baran et Kasparek, 1989a).

Les sites dont nous parlons ci-après sont ceux que Baran et Kasparek (1989a) tiennent pour les plus importantes plages de ponte de Chelonia en Turquie. 


\subsubsection{Kazanli}

Kazanli est situé à quelque $10 \mathrm{~km}$ à $l^{\prime}$ est de Mersin, près de l'extrémité occidentale de la très grande région du delta de Cukurova, formé par les fleuves Tarse (Berdan), Seyhan et Ceyhan, et situé au sud d'Adana. Les basses terres du delta, avec une côte d'environ $130 \mathrm{~km}$, ne se sont peuplées qu'assez récemment, à la suite d'un recul du paludisme. La plage de Kazanli s'étend sur environ 4,5 km; des fabriques de soude et de chrome avoisinent le demi-kilomètre le plus occidental, séparé du secteur suivant d'un demi-kilomètre par un déversoir d'effluents. Ce dernier secteur est adossé à des dunes basses et à une clôture de bambou, qui marquent la limite des terres arables irriguées du delta, et est séparé du secteur suivant, à l'est, par un chantier naval de plage. Le secteur suivant a également environ un demi-kilomètre et est séparé des derniers 2,5 km par un camp de vacances utilisé par la population locale.

Tableau 17. Résumé de données choisies de nidification recueillies sur la plage de Kazanli lors de l'étude de 1988 (Baran et Kasparek, 1989a)

Date Longueur $(\mathrm{km})$ Site Emergences Nids

\begin{tabular}{|c|c|c|}
\hline $\begin{array}{r}20 \text { juin } \\
24 \text { juin } \\
25 \text { juin } \\
26 \text { juin } \\
27 \text { juin } \\
3 \text { juillet } \\
4 \text { juillet } \\
5 \text { juillet } \\
6 \text { juillet } \\
10 \text { juillet } \\
11 \text { juillet } \\
8 \text { août } \\
9 \text { août } \\
11 \text { - } 18 \text { août }\end{array}$ & $\begin{array}{r}70 \\
15 \\
20 \\
5 \\
6 \\
40 \\
6 \\
5 \\
7 \\
4 \\
5 \\
4 \\
1 \\
4\end{array}$ & $\begin{array}{r}55 \\
13 \\
11 \\
4 \\
2 \\
20 \\
2 \\
4 \\
4 \\
3 \\
2 \\
1 \\
1 \\
3\end{array}$ \\
\hline $\begin{array}{l}\text { Totaux: } \\
\text { Plage d'un demi-kilomètre entre fabrique } \\
\text { et chantier naval: } \\
\text { Pourcentage du total: } \\
\text { Densité: } \\
\text { Nids par nuit (pendant la période principale } \\
\text { de juin-juillet): } \\
\text { Nids par km et par nuit (pendant la principale } \\
\text { période de juin-juillet): }\end{array}$ & $\begin{array}{r}156 \\
134 \\
76 \%\end{array}$ & $\begin{array}{l}125 \\
95 \\
47 / \mathrm{km} \\
8 * \\
4,3 *\end{array}$ \\
\hline
\end{tabular}

* Ces chiffres très approximatifs sont calculés d'après les données partielles de juin/juillet tabulées ci-dessus; ils comprennent les nids déjà présents sur la plage le 20 juin, mais non le nombre apparemment décroissant de nids auxquels on pouvait s'attendre pour la période suivant le 11 juillet. 
Langeveld et Sarigül ont participé au travail sur le terrain dans la région de Cukurova, et décrivent (1989) d'une façon assez détaillée le traitement réservé aux tortues et au nidification. (Les chiffres présentés par Langeveld et Sarigül diffèrent légèrement de ceux qu'ont publiés Baran et Kasparek; par souci de consistance, nous retenons ici ces derniers.)

Baran et Kasparek (1989a) soulignent les nombreux facteurs qui influent sur le nidification aux rives de Kazanli. La clôture de bambou du principal secteur de nidification est déplacée en direction de la mer et consolidée en vue d'une protection de l'intérieur arable contre l'inondation, ce qui réduit sérieusement la laisse disponible pour le nidification. Il existe une importante pollution par la lumière des deux fabriques voisines, qui déchargent en outre directement à la mer des effluents industriels apparemment non traités, fortement opaques et de couleur blanchâtre ou verdâtre. Les chalutiers à crevettes pêchent à moins de $50 \mathrm{~m}$ de la plage. Celle-ci est fortement encombrée de déchets de plastiques et de goudron. L'emploi de la plage pour le divertissement ne semble pas avoir d'effet grave sur le principal secteur de nidification, bien que la lumière entraine une désorientation des nouveau-nés dans les secteurs adjacents.

Selon Baran et Kasparek (1989a), des habitants des lieux se rappellent avoir participé à la pêche à la Chelonia au cours des années 60 , alors qu'ils retournaient sur la plage les femelles destinées à être chargées le lendemain matin sur des camions de la compagnie. Les renseignements recueillis par Sella (1982) suggèrent que l'ancienne pêche à la tortue (voir infra) a décimé les populations des environs de Mersin, dont probablement celle de Kazanli, au milieu des années 60 , lorsque la capture des tortues s'est déplacée vers l'est, entre le Seyhan et le Ceyhan. On peut concevoir que la population relativement importante de Kazanli se remette aujourd'hui de l'exploitation assez lourde des années 60 .

\subsubsection{Akyatan}

Cette plage de $21,7 \mathrm{~km}$ est sise sur la côte sud-ouest de la région du delta de Cukurova, son extrémité sud étant voisine de Karatas: le grand lac d'Akyatan se trouve à quelques kilomètres à l'intérieur des terres. Seul un espace de 4 à $5 \mathrm{~km}$ dans le secteur occidental connaît une activité touristique, le reste étant peu utilisé et paraissant modérément touché par la pollution des matières plastiques et du pétrole.

Tableau 18. Résumé de données choisies de nidifications recueillies sur la plage d'Akyatan lors de l'enquête de 1988; on a exclu les données de certaines dates où $l^{\prime}$ inspection $n^{\prime}$ a été que partielle. (Langeveld et Sarigül, rapport préliminaire, 1989)

\begin{tabular}{|c|c|c|c|c|c|c|}
\hline Date & & $\begin{array}{l}\text { longueur } \\
\qquad(\mathrm{km})\end{array}$ & & Site & Emergences & Nids \\
\hline 2 & juin & 18 & Akyatan & (ouest et centre) & 20 & 5 \\
\hline 10 & juin & & Akyatan & (ouest et centre) & 27 & 12 \\
\hline 17 & juillet & & Akyatan & (ouest et centre) & 198 & 72 \\
\hline 3 & juin & 5 & Akyatan & (est) & 0 & 0 \\
\hline 11 & juin & & Akyatan & (est) & 7 & 1 \\
\hline 22 & juin & & Akyatan & (est) & 21 & $>4$ \\
\hline 1er & juillet & & Akyatan & (est) & 6 & 2 \\
\hline 17 & juillet & & Akyatan & (est) & 17 & 6 \\
\hline
\end{tabular}


Langeveld et Sarigül (rapport préliminaire, 1989) offrent une tabulation plus complète des données pour Akyatan, que celle de Baran et Kasparek (1989a); le relevé des empreintes et des nids extrait de Langeveld et Sarigül apparaît au tableau 18. Le nombre total de nids enregistré sur la plage d'Akyatan n'est que légèrement inférieur au total de Kazanli, mais le nidification, à Akyatan, est réparti sur une longueur de plage beaucoup plus grande, la majorité des nids se trouvant cependant dans la partie centrale.

\subsubsection{Samandagi}

Baran et Kasparek (1989a) considèrent ce secteur comme la troisième en importance des plages de ponte de Chelonia, en Turquie. Une bonne partie du rivage oriental de la baie d'Iskenderun est rocheuse; cette plage de $10,3 \mathrm{~km}$ est située à l'extrême sud-est de la Turquie, sur la frontière syrienne. La plupart des nidifications ont lieu le long d'une bande de $5 \mathrm{~km}$ vers le sud, entre un village de vacances et l'embouchure de l'Oronte. On pratique l'extraction de sable à l'extrémité sud de la plage; celle-ci est fortement encombrée de débris, notamment d'objets en plastique.

Tableau 19. Résumé de données choisies de nidification recueillies à la plage de Samandagi lors de l'enquête de 1988 (Langeveld et Sarigül, rapport préliminaire, 1989)

Date Longueur $(\mathrm{km})$ Site Emergences Nids

\begin{tabular}{|c|c|c|}
\hline $\begin{array}{l}19 \text { juin } \\
30 \text { juillet }\end{array}$ & Samandagi & \\
\hline
\end{tabular}

\subsubsection{Plages de ponte de la tortue verte - résumé}

Selon Geldiay (1984: 72, fig. 9), il semble y avoir eu de un à six nids de C. mydas par kilomètre et par jour, au cours de la saison de nidification à Alanya; il y aurait eu moins d'un nid par kilomètre et par jour sur une bande située un peu à l'est d'Alanya, et plus de six nids sur chacune des quatre plages de Mersin, Tuzla, Karatas et Yumurtalik, toutes proches d'Adana, à l'est. Nous ne disposons pas de chiffres précis quant à la longueur de sces plages, mais une extrapolation à partir de ces estimations du taux de nidification suggère qu'à l'époque de l'enquête sur place de Geldiay (fin des années 70, début des années $80)$, il pouvait y avoir chaque année plus d'un millier de femelles reproductrices. Mais on ne connaît pas le détail des inspections sur lesquelles seraient fondés ces calculs de densité du nidification, et certains ont exprimé quelques doutes quant à leur exactitude.

Les résultats de l'inspection des plages par le WWF, la CEE et le DHKD, en 1988, indiquent qu'il y a toujours d'assez importants nidifications de Chelonia dans le delta de Cukurova, entre Mersin et Yumurtalik, bien que les chiffres ne paraissent pas élevés. Langeveld et Sarigül (1989) commentent utilement ces résultats de l'équipe qui a étudié la partie orientale du terrain; les tableaux 20 et 21 résument un choix de données. 
Tableau 20. Données choisies de nidifications recueillies au sud-est de la Turquie (Delta de Cukurova et golfe d'Iskenderun), 1988 (Langeveld et Sarigül, rapport préliminaire, 1989). Les sites sont tabulés selon une séquence ouest-est. La plupart des chiffres sont arrondis au nombre entier le plus proche.

Nids \% du total Nids/km Nids/km/nujt $\begin{array}{r}\text { Période } \\ \text { (maximum) }\end{array}$
$(\max )^{2}$

Kazanli

(plage entière) $\quad 116$

Kazanli

(secteur

principal)

Tarse

Tuzla

Akyatan

(plage entière) 105

Akyatan

(secteur

principal)

Karatas

Agyatan

Yelkoma

Yumurtalik

Yumurtalik

(est)

Dörtyol

Samandagi

(plage entière) 33

Samandagi

(secteur

principal)

\section{5}

1
4

71

3

3

3

15

12

1

33
11.0

4-6. vi

283

.15 10.vi-17.vii

24

7

$.125 . v-31 . v$

$.04 \quad 32 . v-7 . v i$

.04 18.vi-4.viii

.38 16.vii-1.viii

.27 19.vi-30.vii

1

C'est-à-dire le maximum de nids/km/nuit aux dates d'inspection, ce qui ne correspond pas nécesairement à la pointe de toute la saison de nidification.

2 C'est-à-dire la période au cours de laquelle on a enregistré le plus de nidifications. 
Tableau 21. Résumé de données choisies de nidification recueillies au sud-est de la Turquie (delta de Cukurova et golfe d'Iskenderun) entre le 25 mai et le 26 août 1988 (Langeveld et Sarigül, rapport préliminaire, 1989).

Longueur totale des plages inspectées: Total des nids relevés:

Nombre possible de femelles reproductrices*:
$138 \mathrm{~km}$

297 (+ 64 nids possibles) $300-350$

$\begin{array}{llccc} & & \text { km } & \text { nids } & \% \text { du total } \\ \text { Plages mineures: } & \text { Kazanli } & 4.3 & 116 & 39 \\ & \text { Akyatan } & 23 & 105 & 35 \\ & \text { Samandagi } & 10 & 34 & 11 \\ & \begin{array}{l}\text { Yumurtalik } \\ \text { Yumurtalik (E) }\end{array} & 7.5 & 15 & 3\end{array}$

* En supposant trois nids par femelle et par saison, et dans l'hypothèse où chaque femelle pondrait tous les trois ans. 
Tableau 22. Plages de ponte connues pour les tortues marines, et nombre de nidifications, en Turquie. Sources: noms des sites empruntés à Baran et Kasparek (1989a); estimation des densités de nidification de Geldiay et coll., (1982) et Geldiay (1984, texte page 72 et figure 9). Les lieux sont énumérés selon leur ordre ouest-est. Dans les troisième et quatrième colonnes (nids/km/nuit): C.c. = Caretta, C.m. = Chelonia.

\begin{tabular}{|c|c|c|c|c|}
\hline Plage & $\begin{array}{l}\text { Longueur } \\
(\mathrm{km})\end{array}$ & $\begin{array}{l}\text { Nids/k } \\
\text { pour la } \\
\text { C.c. }\end{array}$ & & Femelles/saison \\
\hline Dalyan & 4.0 & $>4$ & - & $200-300$ \\
\hline Dalaman & 7.2 & $?$ & - & \\
\hline Fethiye & 4.8 & $1-4$ & - & \\
\hline Patara (= Kas?) & 11.8 & $1-4$ & - & \\
\hline Finike-Kumluca & 10 & $1-4$ & - & \\
\hline Kemer? & & $1-4$ & - & \\
\hline $\begin{array}{l}\text { Région de Belek } \\
\text { (comprenant } \\
\text { Antalya et Side } \\
\text { ouest) }\end{array}$ & 30 & $>4$ & - & \\
\hline $\begin{array}{l}\text { Région de Kizilot } \\
\text { (comprenant } \\
\text { Side est) }\end{array}$ & 16.6 & $?$ & $?$ & \\
\hline Demirtąs & 7.4 & $?$ & $?$ & \\
\hline Alanya ${ }^{2}$ & 12 & $<1$ & $1-6$ & \\
\hline Gazipasa-Ciftlik & 2.4 & $<1$ & $<1$ & \\
\hline Delta de Göksü & 10.5 & $?$ & - & \\
\hline
\end{tabular}

A. Principales plages de Chelonia selon Geldiay et coll. (1982) et Geldiay (1984)

$\begin{array}{lcc}\text { Mersin } & <1 & >6 \\ \text { Tuzla } & ? & >6 \\ \text { Karatas } & ? & >6 \\ \text { Yumurtalik } & ? & >6\end{array}$

B. Principales plages de ponte de Chelonia selon Baran et Kasparek (1989a)

$\begin{array}{lrll}\text { Kazanli } & 1 & ? & ? \\ \text { Akyatan } & 10 & ? & ? \\ \text { Samandagi } & 5 & ?\end{array}$

Notes pour le tableau 22.

1. Malheureusement, les données sur lesquelles on peut présumer que se fondent ces catégories de dimensions de populations n'apparaissent pas entièrement dans les travaux publiés de Geldiay.

2. Geldiay et coll., (1982) et Geldiay (1984) citent cette plage comme un important lieu de nidification pour les deux espèces; la chose n'a pas été confirmée en 1988 (Baran et Kasparek, 1989a), ce qui suggère un certain déclin.

3. Geldiay (1984); on ne voit pas bien s'il s'agit d'une estimation du nombre total, du nombre de femelles mûres ou du nombre de reproductrices par saison. Le contexte suggère qu'il s'agit très probablement d'une estimation du total des individus non encore adultes et des animaux mûrs.

4. Langeveld et Sarigül (1989). 
On a relevé un total de quelque 300 nids le long de cette côte, pour la plupart à Kazanli et Akyatan (Langeveld et Sarigül, 1989; Baran et Kasparek, 1988). Si l'on suppose que chaque femelle établit trois nids au cours de la saison, et qu'elle niche tous les trois ans quelque 300 femelles pourraient avoir niché sur les côtes sud-est de la Turquie pendant la saison 1988. Le contingent de nidifications relativement mineur qu'indiquent ces données est considérablement inférieur au millier de femelles par an que suggèrent les données de Geldiay pour 1979-1982. Dans un cas comme dans l'autre, la Turquie est de loin la plus importante zone de nidification de $\mathrm{C}$. mydas de la Méditerranée.

\subsubsection{Tendances des populations}

\section{Caretta caretta}

Les données provenant de l'enquête 1988 du WWF, de la CEE et du DHKD (Baran et Kasparek, 1989a) suggèrent un contingent de nidifications de caouannes (Caretta caretta) considérablement inférieur à celui que rapporte Geldiay pour 1979-1982. Il est possible que cette disparité représente dans une certaine mesure des fluctuations saisonnières; mais elle peut aussi être due à des données inexactes. Les données de nidification de Caretta pour 1988 sont difficiles à interpréter, car il semble possible qu'une proportion importante de nidifications n'ait pas été enregistrée; certes, aucune des plages de Caretta n'a été inspectée aussi complètement que les plages de Chelonia de Kazanli et d'Akyatan. Il n'y a donc pas de témoignages probants d'une diminution du nombre de nidifications, bien que celle-ci soit suggérée par les rares données disponibles. Vu les perturbations croissantes que connaissent la plupart des plages de ponte, en raison surtout du développement touristique, on peut s'attendre à un déclin marqué si les sites actuels ne sont pas sauvegardés.

\section{Chelonia mydas}

Il n'existe pas de bonnes données historiques sur le nombre de nidifications antérieurs aux études de Geldiay, qui ont commencé en 1978, même si l'on peut inférer que les chiffres étaient élevés. Baran (1987) a indiqué que "de grands troupeaux" abordaient pour nicher au cours des années 1940 et avant. Il semble y avoir eu un déclin massif du nombre de nidifications, bien que les témoignages en ce sens soient principalement de caractère indirect.

Un très grand nombre de tortues, principalement ou entièrement Chelonia mydas, étaient capturées sur la côte de Mersin-Yumurtalik, au sud d'Adana, entre le début des années 50 et la moitié des années 60 , et des pêcheurs interrogés en 1965-67 affirmèrent qu'il y avait alors de nombreux nidifications (Sella, 1982) (voir section 3.18.6. infra). On a estimé la récolte à quelque 2.000 tortues par an (Sella, 1982). On suppose généralement que le grand nombre de Chelonia capturées indique la présence d'une importante population de tortues nichant sur les plages voisines; mais les nombres dépendent du mode de capture; si les tortues sont prises principalement en mer, il se peut qu'une proportion importante ait consisté en tortues brouteuses ayant pondu sur les plages d'autres pays. Selon Baran (1987), les tortues étaient ramassées en grand nombre sur les plages voisines d'Iskenderun, au cours des années 50 , et cette population de tortues, qui semblait au début inépuisable, a totalement disparu dans l'espace de six à sept ans. De même, Baran et kasparek (1989a) indiquent que des tortues étaient capturées sur la plage de ponte de Kazanli. D'autre part, Sella (1982) donnait à entendre que des tortues étaient prises en mer par des pêcheurs. Il est possible que les deux méthodes aient été pratiquées à divers moments et dans diverses localités. 
Il semble probable que la plupart des tortues exportées de Turquie au cours de cette période, quelle quait été la méthode de capture, étaient prélevées sur les populations reproductrices locales; selon cette hypothèse, il semble s'être produit un déclin massif du nombre de reproductrices entre les années 50 et la fin des années 70 , moment où fut effectué le travail de Geldiay sur le terrain. Cette conclusion est également suggérée par le fait que le centre de capture a dû se déplacer en raison de la diminution des populations locales de tortues (Sella, 1982). En outre, certaines des milliers de tortues capturées au cours des années 20 et 30, au large de ce qui est aujourd'hui le nord d'Israël, pourraient avoir appartenu à la population reproductrice du sud-est de la Turquie et avoir été prises au cours de leurs fameuses migrations vers le nord (Sella, 1982). Une exploitation intensive à l'intention du commerce international semble avoir provoqué la quasi-disparition de la population Chelonia de Turquie (Sella, 1982; Mendelssohn, 1983).

Le nidification tout à fait mineur qu'indiquent les données de 1988 pourrait représenter un nouveau déclin récent de la population Chelonia de Turquie, une variation saisonnière, ou l'effet de données antérieures inexactes.

\subsubsection{Les tortues en mer}

\section{Observations générales}

Lortet (1887) parlait de l'abondance de Caretta (sous son synonyme de Thalassochelys caouana) dans le golfe d'Iskenderun (golfe d'Alexandrie), où l'on pouvait voir s'approcher en même temps cinq ou six individus d'un bateau au mouillage. Gruvel (1931) considérait l'angle nord-est de la Méditerranée, entre la côte cilicienne de la Turquie et la côte de Syrie, y compris le golfe d'Iskenderun, comme un important centre d'abondance de la tortue de mer. Selon lui, Caretta était de loin l'espèce la plus commune en Méditerranée orientale, tandis que Chelonia et Dermochelys étaient relativement rares. On aurait trouvé la caouanne dans le golfe d'Iskenderun, et en particulier dans le golfe de Tarse (au sud de Mersin), mais aussi bien que moins fréquemment - le long de la côte du Levant. Aujourd'hui, Chelonia semble plus commune, dans cette région, que Caretta.

On a noté la présence de caouannes sur la côte égéenne de la Turquie, et elles pénètrent en Mer Noire par la Mer de Marmara (Geldiay, 1984).

\section{Lieux d'alimentation et d'hivernage}

Il y a de bons témoignages (Geldiay 1984) de la présence de rassemblements de Chelonia mydas en hivernage au large de la côte sud-est de la Turquie, d'abord suggérée par la fréquence avec laquelle des tortues de taille adulte sont aperçues lorsqu'elles font surface pour respirer (Geldiay, comm. pers. septembre 1987). Van den Berk et coll. (1988) disent avoir vu un groupe de 15 à 25 tortues dans la baie de Yumurtalik, en avril 1986, et deux couples de Chelonia appariés, le 5 avril 1987. Van den Berk (in litt., 27 juillet 1989) a traversé deux fois la baie de Yumurtalik, dans le golfe d'Iskenderun, le 13 octobre 1988 et, à environ 2 mètres de profondeur dans les eaux claires de l'est du delta de Cukurova, a aperçu de 30 à 40 Chelonia presque adultes (d'une longueur estimée de $40 \mathrm{~cm}$ ) et deux adultes qui fourrageaient probablement sur les abondants lits de zostères. Les 
pêcheurs de la zone auraient aperçu des tortues marines quotidiennement. Des animaux plus jeunes, de 22 à $24 \mathrm{~cm}$ de longueur de carapace, ont aussi été observés dans la région, et ils seraient nombreux en certains points de la côte d'Adana-Yumurtalik, où ils créeraient beaucoup de difficultés aux pêcheurs (Geldiay, 1984). Baran et Kasparek (1989b) notent que dans le delta de Cukurova, l'isobathe de $10 \mathrm{~m}$ se situe entre 1,5 et $6 \mathrm{~km}$ au large, de sorte qu'il y a une vaste zone d'eaux maigres sur un riche substrat alluvial, favorable aux plantes marines que broute Chelonia. Ces observations semblent confirmer les constatations de Gruvel (1933) (bien qu'il ait parlé à ce propos de Caretta), et indiquent que le golfe d'Iskenderun est fréquenté par des tortues hors saison de ponte, y compris par des adultes, des quasi-adultes et des individus plus jeunes.

Cette aire d'alimentation essentielle justifie une enquête plus poussée; celle-ci est urgente car le secteur est grandement exposé du fait de la présence d'industries pétrolières et sidérurgiques aux environs de Yumurtalik, et il est déjà très touché par une forte activité des transports maritimes.

Vu la présence constante de Chelonia adultes ou quasi- adultes dans la région, y compris au cours des mois d'hiver, Geldiay (1984) a suggéré que la population de tortues vertes était résidante. Il a également exprimé $l^{\prime}$ avis que la population de Caretta ne quittait pas les eaux turques de la Méditerranée. Baran et Kasparek (1989b) ont examiné la taille et la distribution spatiale des tortues émergées aperçues au cours de l'enquête de 1988. Toutes les Chelonia, à l'exception d'une seule, ont été trouvées entre Mersin et la frontière syrienne, surtout le long de la côte de Cukurova, où l'on compte le plus de nidifications. La taille de 26 specimens se situait entre environ 10 et $90 \mathrm{~cm}$, longueur courbe de carapace; la plupart étaient des jeunes mesurant entre 20 et $40 \mathrm{~cm}$. La majorité des Caretta aperçues étaient de taille adulte ou quasi-adulte. Baran et Kasparek (1989b) concluent que Chelonia réside dans les eaux adjacentes à leurs plages de ponte dans le golfe de Mersin et le golfe d'Iskenderun, tandis que la plupart des Caretta se dispersent vers des lieux d'alimentation plus distants. On ne sait, en fait, si les tortues reproductrices et les hivernantes font partie de la même population, ou si (comme dans d'autres parties du monde) les tortues aperçues hors saison de reproduction nichent ailleurs; cette dernière hypothèse ne semble guère probable, vu la rareté des nidifications de Chelonia dans la région.

\section{Déplacements}

Geldiay (comm. pers., septembre 1987) indique qu'une Caretta baguée à Dalyan a été recapturée dans les eaux italiennes, et qu'une autre l'a été à Yenifoca, sur la côte égéenne de la Turquie. Deux Caretta baguées lors de nidifications en Grèce ont été retrouvées dans cette dernière zone (Margaritoulis, 1988). Un baguage turc semble avoir été récupéré du Laboratoire de biologie marine d'Eilat, sur le golfe d'Akaba (Geldiay, comm. pers., septembre 1987); mais le fait doit être considéré sans confirmation (aucun détail disponible, et l'on ne voit pas bien comment une tortue baguée en Méditerranée aurait traversé le canal de Suez pour se rendre en Mer Rouge; peut-être l'étiquette seule a-t-elle abouti à Eilat). 


\subsubsection{Menaces}

Les actuelles populations de tortues, qu'il s'agisse de Caretta ou de Chelonia, sont gravement menacées par le développement touristique et par une forte prédation naturelle des oeufs; elles sont également très touchées par les prises accidentelles, et probablement par la pollution (malgré l'absence de preuves directes).

\section{Exploitation}

Gruvel (1931) a signalé l'important commerce international de tortues qu'il a indentifiées comme des Caretta, prises dans le golfe d'Iskenderun et les eaux adjacentes, et destinées à l'industrie de la soupe de tortue d'Angleterre.

Les tortues vertes des eaux turques ont été sérieusement décimées par l'exploitation passée. Selon Sella (1982), on a capturé au large du sud-est de la Turquie, principalement au cours des années 60 , autant de tortues qu'on en a pris entre les deux guerres mondiales au large de ce qui est aujourd'hui la côte septentrionale d'Israël, ce qui correspond à un minimun de 30.000 animaux, soit environ 2.000 par an.

La capture intensive de tortues semble avoir commencé au cours des années 50 et, au milieu des années 60 , une société de pêche avait installé un abattoir à Iskenderoun pour le traitement des tortues achetées de pêcheurs des environs de Mersin. Toute la production était destinée à l'Europe. Mendelssohn (1983) attribue cette initiative commerciale de production de soupe à la tortue à un "Israëlien entreprenant". Au cours de la grande saison de pêche, entre avril et juin, arrivaient chaque jour à Iskenderoun environ 200 tortues ou plus, dont chacune pesait en général de 120 à $150 \mathrm{~kg}$, ce qui donne à penser qu'il s'agissait de Chelonia mydas.

Dans la douzaine d'années qui se sont écoulées entre 1952 et 1965 , on aurait capturé 15.000 tortues dans la seule région de Mersin. La population de tortues a diminué vers le milieu des années 60 , alors que la principale activité de pêche se déplaçait vers l'est de la côte entre les fleuves Seyhan et Ceyhan, au sud d'Adana. On capturait ici tous les jours, en mai 1965, une centaine de tortues ou plus (toutes des Chelonia), de sorte qu'on avait alors pris dans le secteur plus de 10.000 tortues. La chasse à la tortue s'étendait plus à l'est, à Yumurtalik, où l'information recueillie en 1972 suggérait que la prise annuelle se situait à ce moment-là autour de 1.200 tortues (Sella, 1982).

Selon Baran (1987), on capturait un grand nombre de tortues à Iskenderun au cours des années 50 , "dès qu'elles atteignaient le rivage, en général avant qu'elles puissent nicher et pondre"; il ajoute que cette population de tortues, qui paraissait d'abord inépuisable, a entièrement disparu en six ou sept ans (on ne voit pas bien si Baran ne parle que d'une population reproductrice locale, ou de la tortue verte qui niche généralement au sud-est de la Turquie). Baran et Kasparek (1989a) notent que les habitants de Kazanli se souviennent avoir retourné, sur la plage de ponte, les tortues destinées à être ramassées par la société de pêche. Il ne semble y avoir guère de preuve documentaire de la pêche des tortues en mer, bien que Sella (1982) donne à entendre que cette pêche était pratiquée. 
Il est difficile d'évaluer exactement l'impact de ces captures dans la population de Chelonia de Turquie. Notre information actuelle est incomplète, mais le nombre de tortues reproductrices ou observées au large en 1988 semble inférieur à ce que rapportait Geldiay pour 1979-1982, et ne paraît qu'un modeste résidu des populations présentes il y a trois décennies. Il semble probable, comme l'ont dit Sella (1982) et Mendelssohn (1983), qu'une capture intensive à des fins commerciales internationales a conduit presque à l'extinction de la population de Chelonia de Turquie.

\section{Aménagement côtier}

L'aménagement littoral, principalement associé au tourisme international, constitue la menace la plus aiguë et la plus répandue aujourd'hui contre les populations de tortues de Turquie. L'industrie touristique, qui suppose de nouvelles installations telles que les hôtels, pensions, restaurants, routes, et de nouveaux usages civils de l'aéroport de Dalaman, s'est rapidement développée, pour une bonne part sans contrôle, au cours des dernières années. Le rythme de modification des habitats côtiers a été particulièrement rapide au milieu des années 80 , et l'hébergement touristique semble avoir plus que doublé en 1987 et 1988, dans certaines localités, telles que Dalyan et Patara. On trouvera d'autres détails ailleurs, dans cette revue par pays, y compris dans la section 3.18.2.

La côte de Mersin à Yumurtalik, principales zones de nidification de Chelonia en Méditerranée, a connu un très rapide développement touristique depuis quelques années; de même, entre le delta du Göksu, près de Silifke, et Mersin, toutes les plages accessibles de l'intérieur se sont bordées d'hôtels, de pensions et de terrains de camping (van den Berk, Groupe néerlandais de recherche internationale sur les échassiers et la sauvagine, in litt., 23 août 1987). Il existe une vaste zone industrielle à l'est de Mersin, et un tuyau

transportant les déchets de la zone industrielle, à Tasucu (près de Silifke), entre dans la mer tout près de la bonne plage située à l'ouest du delta du Göksu (van den Berk, in litt.). La grande plaire côtière du sud d'Adana fait l'objet d'une culture intensive, principalement de coton, et les eaux voisines reçoivent probablement une forte décharge d'engrais chimiques et de résidus d'antiparasitaires; on ignore les effets d'une telle pollution sur la population de tortues. Des informations récentes, encore inédites, indiquent que la principale zone de nidification de la tortue verte est touchée par la pollution des métaux lourds. Il y a d'importantes prises incidentes de tortues, dont certaines sont libérées vivantes. La topographie de ce secteur de la Méditerranée, conjuguée à un fort trafic maritime, semble faciliter les dépôts intensifs de déchets flottants, notamment de matières plastiques (comme c'est le cas sur de nombreuses plages de Chypre).

\section{Prises accidentelles}

Des pêcheurs de Yumurtalik interrogés par Van den Berk et coll. (1988) ont signalé des prises accidentelles régulières de tortues, par filets, et plus fréquemment par aplets. De rares indices suggèrent que les Chelonia adultes sont rarement ainsi capturées, comme peuvent l'être de jeunes Caretta adultes, bien que la plupart des animaux pris soient des jeunes d'assez grande taille et des quasiadultes. Les estimations du nombre de tortues prises par un petit groupe de pêcheurs variaient entre trois à quatre tortues par jour et 30 à 40 par an. 


\subsubsection{Mesures de protection}

\section{Zones protégées}

Grâce aux patients efforts de la DHKD (Société pour la protection de la vie sauvage) et d'autres, la très importante plage de ponte de Caretta de Dalyan a récemment été déclarée "zone spécialement protégée" par le gouvernement turc. Des panneaux multilingues, à chaque extrémité de la plage, informent les visiteurs de l'importance de celle-ci et indiquent que l'accès en est interdit dès la tombée de la nuit, tout comme l'usage des parasols. Cependant, en juillet 1988 , aucune infrastructure n'était apparente, et $l^{\prime}$ on ne pouvait constater aucun effort pour tempérer l'accès ou restreindre l'usage des parasols; la situation s'est améliorée en 1989, avec la mise en oeuvre, par le DHKD et l'AGA, de programmes de gestion et d'éducation du public. Fethiye a également été désigné comme zone spécialement protégée, fin 1988. Trois autres zones de nidification de tortues marines de la côte turque, celles du delta de Göksü, de Patara et d'Ekincik, ont été désignées comme zones spécialement protégées, comme

$l^{\prime}$ attestait le Journal officiel du gouvernement turc $n^{\circ} 20449$, en date du 2 mars 1990 (Whitmore et coll., sous presse, 1990). Elles jouiront désormais de la même protection que Dalyan et Fethiye, les deux plages de ponte déclarées comme zones spécialement protégées en 1988.

La désignation comme ZSP intéresse aujourd'hui cinq des 17 zones reconnues comme d'importantes aires de reproduction des tortues marines par l'enquête du DHKD, du Fonds mondial pour la nature et de la CEE sur les côtes turques (Baran et Kasparek, 1989a). La nouvelle protection que confère le titre de ZSP en vertu de la loi turque $n^{\prime}$ est pas encore pleinement alignée avec celle des ZSP de la Convention de Barcelone, dont la Turquie est aujourd'hui signataire. La première ZSP turque a été créée à Dalyan, en 1988, avant que la Turquie n'ait ratifié la Convention de Barcelone. La définition officielle de la ZSP turque exige: 1) que les ressources naturelles de la zone en question soient protégées des effets négatifs de l'activité humaine; 2) que tous les projets de développement et de gestion de la zone soient révisés; 3 ) que la zone soit gérée conformément aux accords internationaux et aux lois turques sur l'environnement; 4) que la Direction des ZSP ait pleine compétence pour le règlement de tous les problèmes mésologiques de la zone. Les nouvelles désignations comme ZSP comportent cette exigence légale que toute construction, à l'intérieur de la zone, soit arrêtée et que tout projet d'aménagement soit révisé, à partir du 2 mars 1990 (Whitmore et coll., sous presse, 1990).

L'amélioration de la situation sera désormais entre les mains de la nouvelle Direction des $\mathrm{ZSP}$, à Ankara. Le ministère de la Sylviculture propose actuellement d'agrandir le territoire de la réserve naturelle stricte (RNS) d'Akyatan de manière à y inclure les aires de nidification et d'alimentation des tortues. Les 11 autres zones de nidification non protégées sont toujours menacées par le développement, et souvent par une importante extraction de sable. Néanmoins, le Journal officiel du Département national de la planification du gouvernement turc, (Reg. no 0327, 1989) engageait l'Etat sur un programme à long terme de protection des 17 zones intéressées (Whitmore et coll., sous presse, 1990).

On peut s'attendre à la présence de tortues marines dans les eaux voisines du Parc national de la péninsule de Dilek et du Parc national du littoral d'0lympos; mais on $n^{\prime}$ a eu connaissance d'aucun nidification, et aucune mesure de conservation particulière n'a été prise. 


\section{Observations générales}

Le Comité permanent de la Convention sur la protection de la vie sauvage et du milieu naturel de l'Europe (Convention de Berne), sur l'avis d'un groupe d'experts des tortues marines, a approuvé, au cours de sa réunion de décembre 1987, une recommandation relative à la protection des tortues de Dalyan et d'autres importantes plages de Turquie. Ce texte proposait au gouvernement turc des mesures générales et particulières à prendre pour maintenir les grandes populations de tortues marines relevant de sa juridiction. Vers la fin de 1988, on avait mis partiellement en oeuvre deux des recommandations du Comité permanent: Dalyan avait été déclarée "zone protégée", et les autorités avaient entrepris de réexaminer les projets de développement

touristique interessant d'importantes plages de ponte. On s'est engagé à réexaminer le projet de développement touristique sur la côte méditerranéenne; un suivi - appuyé par le Fonds mondial pour la nature - de l'enquête de 1988 sur les plages sera réalisé en 1990, et permettra de communiquer à diverses autorités des conseils techniques sur la gestion des plages de ponte.

La Turquie a ratifié la Convention de Berne et, pour ce qui est des populations de tortues marines dont elle a la charge, a fait des progrès importants pour remplir les obligations qu'elle a ainsi assumées.

\section{Législation}

La capture de toutes espèces de tortues marines est strictement interdite (Réglementation de la pêche - Gazette officielle du 28 février 1986). Voir supra règlementation des zones spécialement protégées.

\subsubsection{Divers}

Geldiay et coll. (1982) indiquent que des Caretta femelles nichant à Dalyan en 1978-1979, ainsi que des mâles capturés en mer, avaient une carapace mesurant en ligne droite entre 55 et $74,6 \mathrm{~cm}$, et marquaient un poids moyen de $57,5 \mathrm{~kg}$, l'éventail allant de 40 à $75 \mathrm{~kg}$. Baran et Kasparek (1989b) indiquent la longueur moyenne courbe de la carapace des tortues, mesurée lors du nidification pendant l'enquête de 1988: Chelonia, $90,1 \mathrm{~cm}$; Caretta, 75,6 cm.

\subsection{GIBRALTAR}

On ne connaît aucun nidification. Lanteri (1982) rapporte des observations d'un capitaine de bateau (M. Alain Connan) dans la région du détroit de Gibraltar: il se rappelait avoir aperçu, de nombreuses années auparavant, des troupeaux de caouannes qui devaient, selon lui, avoir des activités de reproduction (bien qu'il n'eût connu aucune plage de ponte dans le voisinage). Les tortues de mer auraient été beaucoup plus communes il y a une trentaine d'années (Connan, in Lanteri, 1982). (On trouve d'autres exemples de déplacements massifs de tortues marines dans les sections sur l'Algérie et l'Egypte, supra.) 


\subsection{YOUGOSLAVIE}

On ne connait aucun nidification, et $l^{\prime}$ on sait peu de chose sur la présence de tortues dans les eaux yougoslaves. Une Caretta, baguée et relâchée après recapture par des pêcheurs, au sud-est de I'Italie, a été revue dans les eaux de la Yougoslavie du nord (Argano et Cocco, 1988). Deux Caretta baguées à l'occasion d'un nidification en Grèce ont été retrouvées sur la côte yougoslave (Margaritoulis, 1988). 


\section{POPULATIONS reproductriceS DE MEDITERRANEE}

\subsection{L'ORIGINE DES POPULATIONS reproductriceS DE MEDITERRANEE}

Il est aujourd'hui bien établi que le bassin méditerranéen était isolé de l'océan Atlantique au cours de la phase messinienne du miocène supérieur, il y a 5 ou 6 millions d'années (Hsü, 1972; Maldonado, 1985). Le fait semble avoir été lié aux mouvements tectoniques des plaques qui ont rétréci les détroits faisant communiquer l'Atlantique et la Méditerranée, ainsi qu'à un abaissement mondial du niveau de la mer. La perte d'eau par évaporation en Méditerranée est beaucoup plus importante que l'apport des précipitations, mais le déficit est compensé par une entrée d'eau de l'Atlantique au-dessus du seuil du détroit de Gibraltar; l'ancienne obstruction du détroit, conjuguée à une baisse du niveau de la mer, a empêché cet influx et entraîné l'assèchement de la Méditerranée du miocène. La nature du milieu messinien du bassin est objet de controverse (Maldonado, 1985; Pérès, 1985), mais des témoignages sédimentaires - prédominance des évaporites salines et des marnes provenant d'eaux saumâtres - dénotent la présence de conditions arides et hypersalines propres à faire disparaitre presque à coup sûr les tortues marines de la région. Elles peuvent être revenues dans le bassin lors du rétablissement de conditions normales au passage du miocène ou pliocène, il y a environ 5 millions d'années. Si ce raisonnement est juste, les actuelles populations de tortues marines qui nichent en Méditerranée proviendraient de l'Atlantique, et auraient moins de 5 millions d'années. Si une seule colonisation s'est produite pour chaque espèce reproductrice actuelle, l'étude des rapports génétiques pourrait suggérer l'identité probable de la colonie reproductrice atlantique mère, et ainsi l'épisode messinien de l'assèchement pourrait contribuer à l'établissement d'un calendrier de l'évolution moléculaire chez les tortues marines.

\subsection{COMPARAISON DES TATLLES ET TENDANCES DE LA POPULATION reproductrice}

L'état actuel des populations de tortues marines de la Méditerranée est le produit de siècles d'exploitation locale mineure suivs de décennies d'exploitation locale intensive conjuguée à une importante perturbation des habitats marins et côtiers, et à l'impact massif des prises accidentelles. La plupart des groupes sont probablement des reliquats d'anciennes populations plus prospères. Les deux facteurs démographiques clefs de leur survie sont le nombre de nouveau-nés atteignant la mer, et le nombre de ceux-ci atteignant la maturité; les données sur ces paramètres font complètement défaut pour toutes les populations méditerranéennes. La taille d'une population reproductrice, en chiffres absolus, peut être illusoire; il est possible que les femelles mûres émergent encore pour nicher pendant plusieurs saisons, bien que les cohortes approchant la maturité soient en diminution progressive faute de remplacement, et que la population soit déjà vouée à la disparition.

$\mathrm{L}^{\prime}$ information actuelle sur les plages de ponte est incomplète (voir résumé aux tableaux 23-25). La baie de Laganas (Zante, Grèce) est le seul site important pour lequel existent des données utiles pour plusieurs saisons de ponte consécutives; une information analogue a été recueillie pour les plages de Lara (Chypre), mais est demeurée inédite. On ne sait à peu près rien du nidification des tortues sur les côtes d'Afrique du nord: il est possible qu'une ponte non négligeable y prenne place. 
Dans ces conditions, toute appréciation de l'importance relative des plages de ponte connues ne peut être que provisoire. On peut dire néanmoins, d'après l'information actuelle, que la baie de Laganas, au sud de Zante (Grèce) avec probablement entre 300 et 700 femelles par saison, dans les années récentes, accueille la plus grande concentration de caouannes reproductrices, qui a plusieurs fois la dimension de celle de Dalyan-Iztuzu (Turquie), considérée jusqu'à récemment comme la deuxième par la taille. L'enquête de l'été 1988 a montré que Dalyan ferait plus exactement partie des dix ou vingt premières plages de ponte de Turquie (Baran et Kasparek, 1988a). Seules la Turquie et Chypre sont connues comme accueillant des nombres non négligeables de Chelonia reproductrices, bien que ces nombres, à Chypre, soient très modestes.

L'information disponible sur les tendances de la population évoquées dans les exposés par pays qui précèdent - indique que sans exception les populations reproductrices, de quelque pays qu'elles soient, sont sérieusement menacées, le plus souvent et le plus évidemment par les constructions côtières et par le tourisme international, ou ont fait l'objet de déclin avéré au cours du présent siècle.

La plupart des déclins connus se sont produits dans les années qui ont suivi la deuxième guerre mondiale. La France (Corse), Israël, l'Italie et Malte sont aujourd'hui presque totalement dépourvus de tortues reproductrices. Des caouannes nichaient jadis dans ces quatre pays, et $l^{\prime}$ on voyait des chélonées franches en Israël. Cependant, sauf pour Israël, nous n'avons aucun témoignage probant qu'il y ait jamais eu de fortes populations de reproductrices dans les quatre pays; elles y étaient peut-être relativement modestes. Bien qu'il y ait encore des restes de nidification en Sicile et en quelques sites de l'Italie méridionale, tout comme en Israël, il ne semble guère exister de perspectives d'accroissement des populations dans les conditions actuelles.

Toutes les grandes populations reproductrices connues, en particulier dans la baie de Laganas, au sud de Zante, et le long de la côte méridionale de la Turquie, sont plus ou moins gravement menacées, en particulier par le développement touristique. De très rares populations, par exemple les chélonées franches nichant près de Dipkarpaz, au nord de Chypre, ou les caouannes qui pondent autour de la baie de Kiparissia, dans le Péloponnèse, semblent bénéficier de lieux de ponte peu perturbés (bien qu'elles soient certainement touchées par d'autres facteurs tels que les prises incidentes). Il est indispensable que les grandes plages de ponte et les zones maritimes voisines occupées par les tortues pendant la saison de ponte (secteurs de rassemblement pré-reproduction, zones de reproduction, aires de nidification) soient efficacement protégées.

On est particulièrement inquiet pour la population de tortues vertes qui niche sur les plages entre Mersin et Yumurtalik au sud-est de la Turquie. Cette population, qui est encore la plus nombreuse que l'on connaisse dans le bassin méditerrannéen, a considérablement diminué à partir de l'immédiat après-guerre, période, dit-on, de nidification intense mais aussi de nombreuses captures. Les données recueillies de 1979 à 1982 situent aux alentours de 1000 le nombre de femelles reproductrices à l'époque tandis que pour 1988 , les données préliminaires n'en recensent que 200 à 300 , auquel cas il y a eu récemment une diminution rapide de leur nombre (toutefois, les données pour 1979-1982 ne sont peut-être pas fiables et les fluctuations saisonnières concernant les tentatives de nidification peuvent expliquer en partie l'apparente différence. L'ampleur de l'apparente diminution enregistrée entre 1950 et maintenant signifie que cette population doit être traitée comme une espèce gravement menacée. 
Dans l'ensemble, l'état de conservation des tortues marines nichant en Méditerranée est médiocre, et leurs perspectives de survie à long terme ne sont pas favorables si l'on ne met en oeuvre des mesures rigoureuses de protection et de gestion. Bien que les populations reproductrices de la Méditerranée représentent une proportion pratiquement insignifiante des populations mondiales (il existe ailleurs de nombreuses plages de ponte qui accueillent chacune plusieurs fois le total des Caretta et des Chelonia nichant en Méditerranée), elles constituent une part extrêmement importante du patrimoine naturel et culturel européen, et l'on doit tenir pour tout à fait prioritaires les efforts tendant à assurer leur survie. 
Tableau 23. Résumé de la dimension et des tendances passées et présentes connues des populations reproductrices de caouannes (Caretta caretta) de la Méditerranée

\begin{tabular}{llll} 
Pays & $\begin{array}{l}\text { Dimension Nids par } \\
\text { relative de saison }\end{array}$ & $\begin{array}{l}\text { Femelles par } \\
\text { saison }\end{array}$ & $\begin{array}{l}\text { Tendances de la } \\
\text { population }\end{array}$ \\
& & & \\
\hline
\end{tabular}

Algérie

apparemment peu de nidifications

Chypre

sud

faible

75

nord

faible

50-75?

déclinante, menacée

menacée

Egypte incertain, peut-être important

France

Corse éteinte; jadis faible population seulement?

Grèce

$\begin{array}{llll}\text { Zante forte } & 857-2460 & 300-800 * & \text { déclinante, menacée } \\ \text { Toutes plages forte } & 1400-2500 & <1000 ?\end{array}$

Israël

pratiquement éteinte, dimensions passées incertaines 10-20(1) 0-10* déclinante

Italie

pratiquement éteinte; jadis faible population seulement? 1-2(2) 0-10*

déclinante, menacée

Libye

Kouf N.P. faible 60-65(3) >25(3) ? menacée?

autres sites incertain, pet être important

Malte éteinte; jadis faible population seulement?

Maroc apparemment peu de nidifications

Syrie pas d'information

Tunisie faible? ? ?

Turquie

$\begin{array}{llll}\text { Dalyan moyenne } & 300 \max & 100-150 \star & \text { menacée } \\ \text { Toutes plages forte } & ? & 550-1000(5) & \text { menacée }\end{array}$

Notes du tableau 23: 1. Estimation de 10 à 20 nids pour Caretta et Chelonia ensemble; 2. Estimation pour Lampedusa, seule plage de ponte régulière connue; 3 . Données pour le seul Parc national de Kouf, seules données disponibles; 4. L'estimation faible est fondée sur les données préliminaires d'une étude de 1988, l'estimation forte étant une approximation brute fondée sur le travail effectué en 1979-1982 par Geldiay; l'une et l'autre sont sujettes à révision et la seconde est peut-être même contestable.

Les estimations de taille relative ne s'appliquent que dans le cadre méditerranéen; il n'y a pas de population méditerranéenne de grande dimension par rapport aux normes mondiales. Les estimations de nombres annuels de nidifications - à l'exception de ceux qui sont signalés par une (*) sont des conjectures informées, fondées sur des données incomplètes, plutôt que des estimations établies rigoureusement. Avec l'information disponible actuellement, il est impossible de tenir compte des fluctuations saisonnières naturelles du nombre de nidifications. Voir détails et sources dans les exposés par pays. 
Tableau 24. Résumé de la dimension et des tendances des populations reproductrices passées et présentes connues de chélonées franches (Chelonia mydas) de la Méditerranée

\begin{tabular}{|c|c|c|c|c|}
\hline Pays & $\begin{array}{l}\text { Dimension } \\
\text { relative de } \\
\text { la population }\end{array}$ & $\begin{array}{l}\text { Nids par } \\
\text { saison }\end{array}$ & $\begin{array}{l}\text { Femelles } \\
\text { par saison }\end{array}$ & $\begin{array}{l}\text { Tendances de } \\
\text { la population }\end{array}$ \\
\hline \multicolumn{5}{|l|}{ Chypre } \\
\hline Akamas & très faible & & 25 & $\begin{array}{l}\text { déclinante, } \\
\text { menacée }\end{array}$ \\
\hline nord & faible & & $25-50 ?$ & menacée \\
\hline Egypte & \multicolumn{4}{|c|}{ nidification possible, pas d'information } \\
\hline Israël & $\begin{array}{l}\text { pratiquement } \\
\text { éteinte }\end{array}$ & $0-10 *$ & & $\begin{array}{l}\text { sérieusement } \\
\text { déclinante }\end{array}$ \\
\hline Syrie & \multicolumn{4}{|c|}{ nidification possible, pas d'information } \\
\hline Turquie & forte & & $\begin{array}{l}300-350(2) \\
(<1000 ?)(3)\end{array}$ & $\begin{array}{l}\text { sérieusement } \\
\text { déclinante, } \\
\text { menacée }\end{array}$ \\
\hline
\end{tabular}

\section{Note:}

1. Estimation faible fondée sur des données préliminaires de 1988;

l'estimation forte est une approximation brute découlant du travail effectué en 1979-1982 par Geldiay; l'une et l'autre sont sujettes à révision et la seconde est peut-être même contestable.

Les estimations de taille relative ne s'appliquent que dans le cadre méditerranéen; il $n^{\prime} y$ a pas de population méditerranéenne de grande dimension par rapport aux normes mondiales. Les estimations de nombre annuel de nidifications - à l'exception de ceux qui sont signalés par une (*) - sont des conjectures fondées sur des données incomplètes, plitôt que des estimations établies rigoureusement. Avec l'information disponible actuellement, il est impossible de tenir compte des fluctuations saisonnières naturelles du nombre de nidifications. Voir détails et sources dans les exposés par pays.

Tableau 25. Estimations du total de femelles nichant chaque année en Médi terranée.

\section{Caretta} Chelonia
2000

$300-400$

$(<1000 ?)$

Note: Ces totaux sont très approximatif, ne recouvrent que des

plages de ponte connues, pour lesquelles les données sont très incomplètes, et ils ne tiennent pas compte des fluctuations saisonnières naturelles du nombre de nidifications. Voir détails et sources dans les exposés par pays. 


\subsection{MENACES QUI PÈSENT SUR LES POPULATIONS reproductriceS}

Les témoignages disponibles suggèrent que les tortues marines sont généralement très philopatriques, en ce sens qu'à chacune de leurs saisons de ponte (ordinairement à des intervalles de deux ou trois ans), elles tendent à revenir vers le secteur côtier où elles ont pondu une première fois. Elles ont même une grande fidélité dans le choix du site, et tendent à revenir précisément à la même plage. Au cours d'une saison donnée, elles mettent leurs diverses couvées sur ce même bout de plage. Par conséquent, lorsqu'une plage de ponte devient impropre au nidification des tortues, la population qui y nichait diminue alors ou s'éteint. En mer, les tortues peuvent être victimes de capture et de pollution, mais ces pertes se répartissent en général sur plusieurs classes d'âge et sur les deux sexes, de sorte qu'elles ont sans doute moins d'influence immédiate sur les naissances. Dans ces conditions, les populations $\mathrm{n}^{\prime}$ ont pas la possibilité de se renforcer démographiquement les unes les autres et il est d'autant plus nécessaire de protéger chaque population en particulier.

Beaucoup de données viennent à l'appui de ce modèle dans le cas de Chelonia mydas, mais il est généralement reconnu - et démontré par des faits - que Caretta a moins de fixité dans le choix des sites que Chelonia mydas lorsqu'elle revient nicher au cours d'une saison, ou d'une saison sur l'autre (Bjorndal et Meylan, 1983). Par exemple, une proportion modeste mais représentative de Caretta qui avaient été baguées lorsqu'elles nichaient à Melbourne Beach (en Floride) a été vue nichant de nouveau sur diverses plages (à la fois dans une même saison et dans des saisons différentes). Le plus grand espacement connu entre nidifications était de $550 \mathrm{~km}$, la majorité se situant autour d'une centaine de kilomètres. On est moins informé dans le cas des tortues marines de la Méditerranée. Des femelles baguées à Zante et à Dalyan ont été retrouvées nichant sur les mêmes plages au cours d'années subséquentes, et ailleurs en une seule occasion (une femelle baguée à Zante avait été aperçue à Cephalonia); mais il importe de reconnaitre la possibilité que la population de caouannes nichant dans le sud de la Turquie, par exemple, puisse se partager dans une certaine mesure entre des plages voisines, au lieu d'être strictement attachée à des sites séparés.

Bien que les tortues reproductrices - Caretta plus évidemment que Chelonia - soient capables de changer de plage de ponte en raison de perturbations, il semble qu'un tel changement ne se fasse qu'en faveur de lieux très rapprochés de l'ancienne plage. Il existe d'assez bons témoignages de tels changements, au cours des dernières années, à Zante, où des plages précédemment fréquentées par les tortues reproductrices ne servent aujourd'hui qu'aux touristes, le nidification s'étant concentré sur des portions de plage plus isolées et moins perturbées. Bien que la densité de nidification puisse augmenter en un lieu à mesure qu'elle décroît sur un terrain plus bousculé du voisinage, il faut remarquer qu'il y a de moins en moins de secteurs calmes. Le choix d'une plage de ponte éloignée n'est probablement le fait que d'individus isolés, et il n'y a pratiquement aucune possibilité de voir de nouvelles populations reproductrices se constituer de cette manière en Méditerranée.

Le développement touristique à grande échelle représente la menace la plus généralisée, la plus directe et la plus grave pour les plages de ponte des tortues marines de la Méditerranée. Plusieurs des exposés par pays du présent rapport donnent des exemples des effets de ce développement (par exemple, 3.4. Chypre, 3.8. Grèce, 3.19. Turquie); le reste de la présente section indique en termes généraux les effets néfastes de l'aménagement des plages. 
Plusieurs facteurs liés à I'utilisation et à la modification des plages par l'homme, et souvent à la construction immobilière, ont été reconnus comme influant sur les tortues femelles adultes et sur les nouveau-nés pendant leur séjour sur la plage ou aux environs, ou sont fortement soupçonnés d'exercer une telle influence. Witham (1982-1985) a brièvement parlé de ces facteurs, qui comprennent l'éclairage artificiel, la construction de digues, les structures brise-lames ou anti-érosion, l'extraction de sable, la circulation véhiculaire ou pédestre, l'accumulation de déchets et autres perturbations imputables à l'homme. Il reste peu de parties des côtes septentrionales de la Méditerranée et des grandes îles qui ne soient touchées par le développement touristique, résidentiel, industriel ou agricole.

\subsubsection{Circulation pédestre}

Comme la tortue femelle reproductrice tasse fermement le sable au-dessus de sa nouvelle couvée, il est peu probable que les piétons puissent causer des dommages importants aux nids pendant la période d'incubation des oeufs (Witham, 1984). Néanmoins, lors de l'éclosion et de $l^{\prime}$ émergence, un simple pas sur un nid pourrait faire s'effondrer le sable maintenant desserré, et écraser les nouveau-nés (Witham, 1984).

\subsubsection{Circulation véhiculaire ou animale}

Bien que le fait n'ait pas été quantifié, la plupart des véhicules à moteur semblent capables de causer des dommages aux nids à tous les stades de l'incubation, soit par compaction directe, soit par creusage $d$ 'ornières. Les roues peuvent également écraser des jeunes en route vers la mer (Witham, 1984). Les gros animaux domestiques peuvent aussi causer des dommages aux nids en raison d'un grand poids soutenu par de faibles surfaces de sabot ou de botte cornée. L'emploi de parasols peut causer des dommages par pénétration directe des nids, sans parler de l'ombre portée qui créé un régime de température instable (la température du nid est importante, car le sexe est déterminé selon la température qui prévaut au cours d'une phase critique du développement précoce).

\subsubsection{Embarcations}

Les tortues marines mâles et femelles s'assemblent généralement, au début de la saison de reproduction, dans les eaux voisines de la plage de ponte. Si les tortues qui s'alimentent peuvent être en tout temps victimes de mouvements d'embarcations, notamment les yachts et bateaux rapides à hélices, leur rassemblement pendant les périodes de reproduction les rend particulièrement vulnérables.

\subsubsection{Eclairage artificiel: effet sur les nouveau-nés}

Bien qu'il puisse exister des différences interspécifiques de détail, il a été démontré que l'éclairage artificiel peut désorienter les nouveau-nés qui émergent, et ce dans toutes les espèces de tortues étudiées, y compris Caretta et C. mydas (Mortimer, 1982;

Raymond, 1984). 
Les résultats de nombreuses enquêtes (passées en revue par Raymond, 1984) indiquent que lorsque les tortues nouveau-nés émergent du nid (généralement la nuit), leur orientation vers la mer dépend largement d'une réponse visuelle à des intensités lumineuses relatives, avant tout à l'horizon. Sur des plages de ponte calmes, un mouvement vers l'horizon plus lumineux sera généralement un mouvement vers la mer; lorsqu'un éclairage artificiel atteint la plage, les nouveau-nés sont fréquemment désorientés et perdent beaucoup de temps à errer sur le sable ou à se diriger vers la terre, du côté de la source lumineuse. Ils sont alors plus exposés aux prédateurs (par exemple, aux crabes de sable), et peuvent mourir de dessication, le jour suivant. On a largement et fréquemment constaté ce phénomène sur de nombreuses plages de ponte de par le monde, qu'atteignent les éclairages artificiels (Raymond, 1984). Bien que nous ayons peu de données quantitatives, il est possible qu'une importante proportion des nouveau-nés soit ainsi perdue chaque année. Dans une étude récente sur une grande plage de ponte de Floride, on a noté une diminution de la désorientation des nouveau-nés à la suite des restrictions imposées aux éclairages du bord de mer (Ehrhart et witherington, 1987).

\subsubsection{Eclairage artificiel: effet sur les femelles reproductrices}

Il semble que la capacité des femelles à s'orienter vers la mer après nidification soit sujette aux mêmes conditions que pour les nouveaunés. Comme ceux-ci, les tortues peuvent être induites à s'orienter vers une source lumineuse, par exemple une torche ou un éclairage artificiel du côté de la terre (qu'il s'agisse de lumière directe ou de la lueur lointaine d'une ville éclairée); on en voit souvent qui sont ainsi égarées vers l'intérieur plutôt que d'être en route vers la mer, et qui peuvent être piégées ou mourir de chaleur ou d'épuisement.

La désorientation des femelles reproductrices ne semble pas aussi fréquente que celle des nouveau-nés. Il semble que cela soit dû, dans une large mesure, au fait que les femelles reproductrices - au moins de certaines

espèces et en certaines périodes - n'émergent pas sur des plages artificiellement éclairées (Mortimer, 1982). Sur la plage de Melbourne, en Floride, C. mydas préfère nettement les secteurs dépourvus d'éclairage artificiel, et la gêne au nidification disparaît lorsqu'on élimine les nuisances lumineuses (Ehrhart et Witherington, 1987).

Si un éclairage mouvant dissuade les femelles de toutes les espèces de tortues marines de nicher, certains faits indiquent qu'un éclairage stationnaire, en certains cas, n'a que peu d'effet démontrable sur le nidification des caouannes (Caretta caretta) (Mortimer, 1982). Par exemple, Mann (1977, cité par Witham, 1982) observe que de nombreuses Caretta nichant en Floride continuent de le faire sur des plages densément construites, proches de brillants éclairages, avec de hauts bấtiments et une forte activité humaine, même lorsque certaines des plages plus naturelles des environs $n^{\prime}$ accueillent que peu de reproductrices. La situation

est différente de celle qui a généralement cours sur les côtes du sud-est des Etat-Unis (voir infra). Witham (1982) suggère que dans certains cas il semble que ce soient les activités humaines, impliquant bruits et lumières mouvantes, plutôt que le seul éclairage, qui réduisent l'activité de nidification. 
Dans l'ensemble, il apparaît que sur des plages à d'autres égards équivalentes, le nidification se concentre dans les secteurs les moins affectés par les phénomènes associés à l'aménagement. C'est

certainement le cas de Zante, où la plus grande partie de la ponte se fait aujourd'hui à Sekania, la plage la plus isolée, et où il y a peu d'activité dans le secteur de Laganas proprement dit, qui était naguère très fréquenté (Margaritoulis, 1982; Sutherland, 1984). La diminution du nidification des tortues à l'île d'Hutchinson (Floride) est attribuée, entre autres facteurs, à une urbanisation croissante accompagnée d'une augmentation des niveaux d'éclairage et d'activité humaine (Worth et Smith, 1976). De même, Dean et Talbert (1975) ont noté une diminution de l'activité de nidification de Caretta sur les plages de Caroline du sud, en raison de la construction et de l'absence de limitation d'éclairage, et ont observé que le nidification s'accroît au contraire sur les plages non perturbées. Shabica (1982) cite plusieurs cas où le nidification des tortues, au sud-est des Etats-Unis, a augmenté sur des plages protégées, les femelles ayant apparemment changé de lieu de ponte en raison de l'aménagement des plages précédemment fréquentées. 


\section{LES TORTUES RN MER}

\subsection{VA-ET-VIENT ENTRE MÉDITERRANÉE ET ATLANTIQUE}

On n'a que deux exemples documentés d'entrée en Méditerranée depuis l'Atlantique: (1) une Lepidochelys kempii - espèce qui ne se reproduit que dans le golfe du Mexique et que l'on a très souvent vue dans les régions nord-ouest et nord-est de l'Atlantique - a été signalée dans les eaux maltaises (Brongersma et Carr, 1983); (2) une Caretta caretta qui avait eu un départ protégé à l'île Padre (Texas), a été recapturée quatre ans plus tard au sud-est de l'Italie.

Nonobstant, des témoignages indirects et des observations occasionnelles de tortues en mer, près du détroit de Gibraltar, suggèrent que des tortues entrent régulièrement en Méditerranée, et peut-être en assez grand nombre. Par exemple, des tortues-cuir (Dermochelys coriacea) ont été souvent signalées, et à de nombreux endroits, en Méditerranée, et de petits groupes ont été vus aux environs du détroit de Gibraltar; aucune plage de ponte n'a été confirmée dans le bassin, et l'on peut supposer que la plupart, sinon toutes les tortues-cuir de la Méditerranée sont des immigrantes, appartenant probablement aux populations reproductrices de l'Atlantique.

Parlant des émigrations lointaines de jeunes tortues, Carr (1987) estimait raisonnable d'écarter l'idée que les jeunes tortues marines vues dans l'Atlantique oriental, et en particulier aux abords des îles, viennent de la Méditerranée. Les colonies reproductrices connues de la Méditerranée semblent trop modestes pour fournir des populations aussi nombreuses, et un mouvement vers l'ouest serait probablement contré par de forts courants de surface entrant de l'Atlantique en Méditerranée, par-dessus le seuil de Gibraltar. On a estimé le mouvement de ces eaux de surface à près d'un million de mètres cubes par seconde, et le courant en sens contraire se situe à une profondeur de 80 à $100 \mathrm{~m}$ (Estrada et coll., 1985). Carr a suggéré que "la Méditerranée, au lieu de fournir des tortues à la population atlantique, accueillait probablement les égarées des migrations transatlantiques de tortues américaines"; il pense en outre que certaines des Caretta de 20-40 cm trouvées près des Açores pourraient avoir dévié, du courant principal Açores-Canaries, dans la branche qui se dirige vers l'est à travers le détroit de Gibraltar. Les caouannes, principalement non adultes, que l'on trouve près des Baléares sont généralement un peu plus développées que celles que l'on signale autour des Açores (données de Mayol et Castello Mas, exploitées par Carr, 1987).

La distribution de tortues de taille de plus en plus grande, dans cinq échantillons de population: (1) nouveau-nés avancés et jeunes des eaux du sud-est des Etats-Unis, (2) individus des Açores, (3) individus des Baléares, (4) quasi-adultes du sud-est des Etats-Unis, et (5) tortues adultes et reproductrices des plages de Floride, pourrait correspondre en partie à des étapes successives d'un itinéraire de développement et de migration de Caretta dans l'Atlantique nord, gouverné et stabilisé par les grands courants océaniques (Carr, 1986, 1987). 
On ne saurait dire si la Caretta des Baléares et les tortues d'origine atlantique qui se trouvent ailleurs dans le bassin méditerranéen sont définitivement détournées de cet itinéraire présumé, ou sont capables de quitter le bassin et de retourner finalement à leur lieu de reproduction de 1'Atlantique occidental. Selon Brongersma (1982:414), on sait que les tortues marines traversent le détroit de Gibraltar dans les deux sens, mais si l'on a des témoignages directs (cités supra) que des tortues de l'Atlantique entrent en Méditerranée, il ne semble pas $y$ en avoir que des tortues migrent dans $l^{\prime}$ autre direction, de la Méditerranée à l'Atlantique. Il semble tout à fait possible que le détroit de Gibraltar constitue un filtre dont l'action se traduirait par un gain net pour les populations de tortues de la Méditerranée.

La mesure dans laquelle un échange se produirait effectivement serait un précieux élément d'information: le problème des prises incidentes massives en Méditerranée perdrait de sa gravité pour les modestes populations reproductrices du bassin, dès lors que la pression serait supportée par des populations américaines aussi bien que par les méditerranéennes. Argano (1979) conclut que les tortues victimes de prises incidentes en Méditerranée occidentale proviennent principalement de populations nichant en Méditerranée orientale et, en partie, de celles de l'Atlantique. Il reste à déterminer quelles seraient les contributions relatives de ces deux groupes. On pourrait procéder à une telle appréciation si les études actuelles des rapports génétiques (ADNmt) entre des échantillons de Caretta de plages de ponte d'Amérique du Nord et de Grèce (Meylan, comm. pers.) étaient étendues à des échantillons de jeunes tortues capturées en mer autour des Baléares.

On peut même imaginer que certaines tortues d'origine atlantique pourraient atteindre la maturité en Méditerranée et se reproduire par la suite dans le bassin. Ce n'est certainement pas ce à quoi l'on s'attendrait si - comme on le dit fréquemment - les tortues adultes retournaient régulièrement à leur plage natale pour la ponte; mais ce serait possible si des femelles récemment parvenues à maturité trouvaient leur première plage de ponte en se joignant à des groupes de reproductrices déjà constitués. On pourrait mettre cette hypothèse à l'épreuve si l'on trouvait le moyen de baguer efficacement des Caretta quasi adultes de l'Atlantique, peut-être de celles que l'on rencontre en mer autour des illes de l'Atlantique oriental.

On peut lier les groupes de Caretta de Méditerranée occidentale à l'existance d'un "front" permanent (une convergence de courants descendants produisant une accumulation de nourriture et d'autres objets flottants) qui s'étend, en passant par les Baléares, d'Almeria, en Espagne, à Oran, en Algérie (Carr, 1987).

\subsection{MOUVEMENTS DES TORTUES EN MÉDITERRANÉE}

Alors que les migrations lointaines de femelles reproductrices entre secteurs d'alimentation et plages de ponte est un phénomène bien établi pour plusieurs populations de tortues marines, en particulier Chelonia mydas, la distribution et les mouvements des mâles et de différents groupes d'âge sont pratiquement inconnus. L'information sur les mouvements dispersifs et migratoires des tortues marines de la Méditerranée est très rare et généralement peu probante. 
La plupart des données se rapportent à la population de Caretta nichant à Zante (Grèce), qui a fait l'objet d'un des très rares programmes de baguage de la Méditerranée, et qui a fourni le plus riche échantillon de tortues baguées du bassin. Les recaptures de femelles baguées (Margaritoulis, 1988) ont montré que cette population se dispersait sur de très grands espaces en Méditerranée centrale, jusqu'à la Sardaigne à l'ouest et à la Turquie à l'est, à l'Adriatique et à l'Egée au nord, à la Tunisie et à la Libye au sud.

Des caouannes capturées en mer au large de l'Italie du sud-est, baguées et relâchées, ont été recapturées dans le même secteur général où l'on a recapturé des individus de la population de Zante (bien que l'on n'ait pas signalé à ce jour de reprise aussi loin à l'ouest que la Sardaigne, et à l'est que la Turquie) (Argano et Cocco, 1988). Bien qu'une partie de l'échantillon bagué dans les eaux italiennes puisse avoir son origine sur les plages de ponte grecques, relativement proches, 1'origine des tortues de l'échantillon italien, à deux exceptions près, est inconnue. Les exceptions sont une tortue baguée à Zante et recapturée au sud-est de l'Italie, et, ce qui est plus intéressant, une Caretta qui avait eu un départ protégé au Texas et fut recapturée quatre ans plus tard au sud-est de l'Italie (Manzella et coll., 1988).

??? Laurent et coll. (sous presse, 1989) signalent la capture dans le golfe de Gabès (Tunisie) de Chelonia mydas bagué à Chypre. Il paraît être le seul cas noté de déplacement de longue distance dans le bassin. Il y a de témoignage indirect d'une migration saisonnière des tortues, y compris Chelonia, le long de la côte lévantine de la Méditerranée orientale (voir section 3.8.5). (English p. 77) ???

Selon Geldiay (1984), les populations de tortues (tant Caretta que Chelonia) nichant sur la côte turque y sont résidantes et ne quit tent pas les eaux turques. Alors que des $C$. mydas jeunes, quasiadultes et adultes sont présentes dans le golfe de Mersin et celui d'Iskenderun pendant une grande partie de l'année, et sont probablement néés sur les plages de ponte turques, Caretta semble moins fréquemment observée et, selon Baran et Kasparek (1989b), se disperse généralement vers des eaux lointaines.

Aucune des tortues baguées sur une plage de ponte méditerranéenne n'a été trouvée jusqu'ici parmi le grand nombre de tortues capturées accidentellement par des pêcheurs autour des Baléares. Une forte proportion de ces tortues sont des quasi-adultes, de sorte qu'elles ne pouvaient avoir été baguées sur une plage de ponte, mais - comme nous l'avons dit - il est tout à fait possible qu'une partie importante des Caretta des Baléares soit de provenance atlantique.

Comme le note Laurent (sous presse), la plupart des Caretta aperçues dans les eaux méditerranéennes sont des isolées ou font partie de très petits groupes. Les grands troupeaux de tortues (cités dans les exposés par pays, supra), y compris de Caretta, ont été signalés trop rarement pour permettre de tirer des conclusions nettes sur les mouvements collectifs. Une exception possible serait la Caretta de Méditerranée occidentale et du détroit de Gibraltar, où I'en pense qu'il pourrait exister des échanges entre Atlantique et Méditerranée (voir supra). 
Les mouvements qu'indiquent les témoignages dont nous disposons ne sont pas simples et, à la différence de ce qui existe pour d'autres populations de tortues marines dans le monde, on ne voit pas en Méditerranée de populations reproductrices migrant régulièrement et uniquement entre la plage de ponte et une zone $d^{\prime}$ alimentation distincte. Une très large portion de la Méditerranée centrale semble constituer l'espace sans nidification de la population de Caretta de Zante, et sans doute de Caretta d'autres plages de ponte méditerranéennes, probablement même de tortues des plages de ponte de l'Atlantique. Certains indices tendraient à montrer d'autre part que des éléments des populations de Chelonia reproductrices de Turquie, et peut-être leur totalité, résident en permanence dans les eaux turques. La distribution des groupes d'âge, des sexes et des diverses populations reproductrices est presque entièrement inconnue.

Les mouvements au sein même des populations, récemment démontrés par les résultats d'une longue étude des caouannes de l'Atlantique occidental (Enwood, 1987), sont peut-être un phénomène plus général, et une caractéristique des caouannes de Méditerranée. Enwond donne de bons témoignages à l'appui du fait que les femelles adultes nichant autour du Cap Canaveral (Floride) sont des visiteuses de passage dans la région, à des intervalles de deux ou trois années, et résident ailleurs au cours des années où elles ne nichent pas. Les mâles adultes ne migrent pas avec les femelles mais semblent demeurer dans le voisinage des plages de ponte, toute l'année durant. Les tortues quasi adultes se déplacent selon l'opportunité le long de la côte atlantique, allant vers le nord en été et vers le sud en hiver, une population résidante de ce type hivernant dans la région de Canaveral (où un grand nombre d'entre elles, enfouies dans la vase ont été draguées du fond de mer).

\subsection{ZONES D'BIVERNAGE ET D'ALIMENTATION}

Un nombre assez important de caouannes, en état apparent de torpeur, ont été prises dans des filets de pêche au-dessus de fonds vaseux de l'Adriatique centrale et septentrionale, et l'on a suggéré que ca serait là un important secteur d'hivernage ou d'"hibernation". De même a-t-on pensé que les tortues (tant Caretta que Chelonia) présentes dans les eaux turques pendant les mois d'hiver pourraient hiberner dans certains secteurs. Il importe de réunir davantage de renseignements sur le phénomène de la dormance hivernale des tortues marines de la Méditerranée, car les tortues dormantes sont des victimes désignées des prises incidentes par chalut.

On a noté que les caouannes des eaux américaines septentrionales réagissaient différemment à la baisse de la température de l'eau; des individus de même espèce, voire d'une même population, migrent, tandis que d'autres hibernent (Ogren et Mac Vea, 1982). En Méditerranée, il se peut que certaines caouannes se trouvant en Adriatique centrale et septentrionale, par exemple, soient forcées par une baisse de la température de l'eau d'y demeurer, tandis que d'autres sont en mesure de migrer vers le sud pour atteindre les secteurs d'alimentation des côtes nord-africaines.

On rencontre régulièrement, dans le golfe de Gabès, de nombreuses caouannes, y compris quelque $40 \%$ des tortues recapturées après avoir été baguées sur les plages de ponte grecques. La plupart des recaptures de tortues baguées en Grèce ont lieu pendant les mois d'hiver. Bien que le nombre élevé des retours de bagues doive refléter dans une certaine mesure l'intensité de la pêche à la tortue dans les eaux tunisiennes, il paraît certain que le golfe de Gabès soit une zone majeure d'alimentation (et d'hivernage) pour les caouannes de Méditerranée (voir section 3.17.3.). 
Il apparaît que certains secteurs marins occupés par des tortues pendant l'hiver sont également des sites actifs d'alimentation (comme précisément le golfe de Gabès), tandis que d'autres zones (comme l'Adriatique centrale et septentrionale, et les eaux voisines du sud de la Turquie) sont peut-être des lieux où les tortues hivernent en état de torpeur. Le degré d'activité des mois d'hiver est sans doute lié directement aux températures marines et aux possibilités d'alimentation. Les caouannes vues en mer, dans la partie nord-ouest de la Méditerranée (Corse), quittent cette région assez soudainement à la fin de l'été, peut-être pour trouver des eaux plus chaudes au sud et à l'est. Peut-être faut-il voir là la tendance prédominante des caouannes de la Méditerranée.

Des tortues vertes de diverses catégories d'âge, et des Caretta moins fréquemment, sont aperçues en hiver dans le golfe de Tarse (Mersin) et celui d'Iskenderun (voir section 3.18.5. supra). Gruvel (1931) a attiré l'attention sur cette région en tant que centre majeur d'abondance de tortues, et le fait semble confirmé par des observations plus récentes (voir section sur la Turquie, 3.19.5). On a suggéré que, dans le passé, des tortues migraient régulièrement en direction du nord, le long de la côte du Levant, vers les plages de ponte de la Turquie. On ne saurait dire, cependant, si les Chelonia reproductrices de la région ne comprenaient pas deux groupes différents s'alimentant dans des secteurs tout à fait séparés. On dit qu'il y avait naguère un bon nombre de chélonées franches le long des côtes méditerranéennes de la Turquie, riches en plantes marines (aliment important); mais, ici encore, l'activité humaine a joué son rôle.

Dans l'ensemble, nous n'avons que peu d'information détaillée sur la distribution des tortues dans les eaux méditerranéennes. Presque tous les faits signalés le sont à l'occasion d'activités humaines, généralement de pécheurs. Aussi, les observations sont-elles limitées aux actuels lieux de pêche, à des eaux ayant une certaine profondeur et situées à quelque distance des côtes. Il semble que l'on rencontre des caouannes en bon nombre autour des Baléares, de l'Italie méridionale, de Malte et de la Tunisie, et peut-être un peu moins nombreuses ou plus saisonnièrement autour de la Corse et de la Sardaigne. Dans une certaine mesure, c'est probablement là le reflet de la distribution des activités de pêche autant que de la distribution des tortues; mais il est possible que ces dernières et les pêcheurs cherchent leurs ressources alimentaires dans les mêmes eaux et pour les mêmes raisons. A la notable exception du golfe de Gabès, au sud de la Tunisie, on ne peut guère, avec l'information dont nous disposons actuellement, formuler que des observations générales sur les secteurs d'alimentation préférés des tortues.

\subsection{LES TORTUES EN MEDITERRANEE ORTENTALE}

Gruvel (1931) considérait l'angle nord-est de la Méditerranée, entre la côte cilicienne de la Turquie et la côte de Syrie, y compris le golfe d'Iskenderun, comme un centre d'abondance des tortues marines. On disait trouver la caouanne dans le golfe d'Iskenderun et celui de Tarse (au sud de Mersin) en particulier, mais également, quoique moins fréquemment, le long de la côte levantine. Un passage du chalut dans le golfe d'Iskenderun ramenait jusqu'à une soixantaine de Caretta, que I' on exportait vers l'Angleterre, via Chypre et Alexandrie (Gruvel, 1931). 
Il faut noter que, selon des autorités précédentes, telles que Lortet $(1883,1887)$ et Gruvel (1931), la caouanne ou Caretta Caretta (= Thalassochelys caouana ou $\mathrm{T}$. caretta) était de loin la tortue la plus abondante en Méditerranée orientale, et l'espèce la plus utilisée dans le commerce international de la tortue des années 1920; Chelonia et Dermochelys étaient relativement rares. En revanche, selon Hornell (1935) et Sella (1982) Chelonia mydas était pratiquement la seule espèce recherchée par la pêche au large du nord d'Israël pendant les années 20 et 30 , ainsi que dans les golfes de Tarse et d'Iskenderun, en Turquie, pendant les années 50 et 60 . Hornell a exploré ce qui est aujourd'hui Israël septentrional, tandis que Gruvel a inspecté la côte syrienne et ce qui est devenu le Liban, et il est concevable que Chelonia ait été alors l'espèce prédominante dans la première région nommée, et que Caretta l'ait été dans la seconde. La possibilité d'"erreur sur la personne" ne peut être absolument exclue. Hornell connaissait certainement bien Chelonia par son expérience de la pêche dans d'autres régions. Les longueurs cités par Lortet (1,5-2 m) et par Gruvel (1,1-1,2 m) paraissent excessives, mais sembleraient plutôt proches de la taille de Chelonia que de celle de Caretta; la longueur moyenne courbe de la carapace de la Caretta nichant aujourd'hui en Méditerranée est proche de $0,8 \mathrm{~m}$ (peut-être mesurait-on à l'époque carapace et tête). Si les divers auteurs ont correctement identifié les espèces ont doit conclure à un renversement radical de l'abondance relative des espèces.

\subsection{PRISES ACCIDENTELLES}

Un très grand nombre de tortues, principalement des Caretta, se prennent dans des agrès de pêche, en Méditerranée, généralement - mais non exclusivement - à l'occasion d'activités de pêche visant d'autres espèces. Le nombre de tortues capturées n'est pas égal à celui des tortues tuées en raison d'une prise incidente; les aplets sont généralement relevés à des intervalles fréquents, et les tortues qui y sont accrochées ne sont pas nécessairement noyées (elles sont souvent relâchées, et l'on a vu, semble-t-il, que certaines tortues peuvent être hameçonnées plisieurs fois). Les tortues prises dans des filets qui (surtout $s^{\prime} i l s$ sont tendus pour des langoustes) peuvent rester en place pour des périodes beaucoup plus longues, sont plus souvent noyées. Les données actuelles ne permettent pas une estimation sûre de la mortalité réelle des tortues.

Roberto Argano (1979) a mené une enquête préliminaire auprès des pêcheurs d'une cinquantaine de ports des côtes d'Espagne (continentale), d'Algérie, de Tunisie et d'Italie (voir tableau 9). Il en a conclu qu'un minimum de 5.000 tortues étaient tuées chaque année en Méditerranée occidentale, la plupart à l'occasion de la pêche d'autres espèces, notamment en raison de l'emploi d'aplets pour l'espadon et, par exemple, de chaluts dans le détroit de Sicile. Argano (1979) indique que quelques-unes seulement des tortues capturées sont tuées, ce qui implique sans doute que le total des tortues capturées dépasse largement 5.000. Les animaux que l'on prend pour les empailler ou pour en apprêter la carapace (dans les deux cas, comme souvenirs pour touristes ou curiosités) sont généralement tués sur-le-champ, tandis que ceux que l'on destine à l'alimentation (Italie méridionale, Espagne, Tunisie) sont souvent gardés vivants dans le port (comme on le voit à Capraia et à l'île de Ponza) ou à bord ( $\mathrm{Sfax}$ ), jusqu'à ce qu'on en ait besoin. Selon Argano, la vente de chair de tortue n'est presque partout qu'occasionnelle, et l'importance économique de ce mets est négligeable. Ce ne semble pas le cas pour la Tunisie, où les tortues sont prises et vendues en grand nombre. 
Des enquêtes plus récentes ont permis de meilleures estimations de la prise totale de tortues, bien qu'on ne puisse toujours apprécier la mesure dans laquelle la prise totale équivaudrait à la mortalité totale. Ont été particulièrement remarquables deux études faites autour des Baléares, desquelles il ressort que de 16.000 à 20.000 Caretta sont capturées chaque année par les pêcheurs aux aplets, un grand nombre d'entre elles étant relâchées, mais quelques-unes pouvant mourir par la suite. Il semble que des tortues aient été capturées plusieurs fois. D'autre part, la prise totale, dans les eaux tunisiennes, semble aujourd'hui beaucoup plus importante que ne le suggère Argano, et dans la plupart des cas les tortues semblent être l'espèce visée, car ces animaux sont vendus sur les marchés de la côte et largement utilisés dans l'alimentation.

Tableau 26. Estimation provisoire du nombre de tortues tuées à 1'occasion de la pêche en Méditerranée occidentale, fondée sur des entretiens organisés en 1978 par R. Argano (Argano, 1979). On suppose que la plupart des tortues étaient des Caretta, et elles sont généralement tuées à l'occasion d'activités de pêche visant $d^{\prime}$ autres espèces.

Nombre de tortues tuées annuellement

$10-100 \quad 100-500 \quad 500-1000>1000$

Algérie: total $<100$

Italie: total $350->3000$

$\begin{array}{ll}\text { Gallipoli } & \text { Iles éoliennes } \\ \text { Palerme } & \text { Trapani \& } \\ \text { Port Empédocle } & \text { Marsala } \\ \text { Licate } & \text { Sciacca } \\ \text { Lampedusa } & \end{array}$

Espagne: total environ 650-3750

$\begin{array}{ll}\text { Alicante } & \text { Castellón } \\ \text { Almeria } & \text { Altea } \\ \text { Adra } & \text { Cartagéna } \\ \text { Motril } & \text { Aguilas } \\ \text { Málaga } & \text { Estepona } \\ \text { Tarifa } & \text { Algeciras }\end{array}$

Tunisie: total environ 1000 $>3000$

Sousse 
Tableau 27. Résumé de la prise annuelle de tortues en Méditerranée (voir sources des données dans les exposés par pays). Abréviations: deuxième colonne, $\mathrm{A}=$ prise accidentelle, $\mathrm{T}=$ espèces visées; dans la troisième colonne, $L L=$ aplets, $N=$ filet; dans la quatrième colonne,

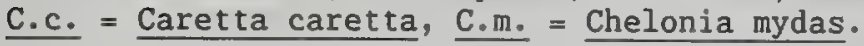

\begin{tabular}{|c|c|c|c|c|}
\hline Région & $\begin{array}{l}\text { Prise accidentelle } \\
\text { ou espèce visée }\end{array}$ & $\begin{array}{l}\text { Méthode de } \\
\text { pêche }\end{array}$ & Espèce & $\begin{array}{l}\text { Nombres par } \\
\text { an }\end{array}$ \\
\hline Algérie & A & LL, N & C.c. & 500 \\
\hline Corse & A & $N(L L)$ & C.c. & $\begin{array}{l}\text { nombre assez } \\
\text { élevé }\end{array}$ \\
\hline Chypre & A & $\mathrm{N}$ & C.c., C.m. & petit nombre \\
\hline Egypte & $\mathrm{T}$ & $?$ & $?$ & $\begin{array}{l}\text { probablement } \\
\text { nombre élevé }\end{array}$ \\
\hline Grèce & A & LL, $N$ & C.c. & nombre assez élévé \\
\hline Israël & $A$ & $\mathrm{~N}$ & $?$ & $\begin{array}{l}\text { captures } \\
\text { occasionnelles }\end{array}$ \\
\hline $\begin{array}{l}\text { Italie } \\
\quad \text { continentale }\end{array}$ & A & LL & c.c. & $\begin{array}{l}250-1000(1) \\
16000(2)\end{array}$ \\
\hline Lampedusa & A & LL & C.c. & $150-300(3)$ \\
\hline Sardaigne & $\mathrm{A}, \mathrm{T} ?$ & $?$ & C.c. & ? \\
\hline Sicile & $A, T$ & LL, N & C.c. & $\begin{array}{l}>600(4) \\
>5000(5) \\
>1000(6)\end{array}$ \\
\hline Malte & A, T & $\begin{array}{l}\text { LL, N } \\
(\mathrm{etc} .)\end{array}$ & c.c. & $\begin{array}{l}1000-3000 \\
500-600(7)\end{array}$ \\
\hline Maroc & A & LL & C.c. & $>3000(8)$ \\
\hline $\begin{array}{l}\text { Espagne } \\
\text { continentale }\end{array}$ & A & LL, N & C.c. & $>1000$ \\
\hline Iles Baléares & A & LL & C.c. & 16000 \\
\hline Tunisie & $\mathrm{T}$ & $\mathrm{N}$ & C.c. & $4000-5500(9)$ \\
\hline $\begin{array}{c}\text { Turquie } \\
\ldots . . .\end{array}$ & A & LL, N & C.c., C.m. & $?$ \\
\hline \multicolumn{4}{|c|}{$\begin{array}{l}\text { Prises totales annuelles estimées: } \\
\text { non équivalentes à la mortalité totale(10): }\end{array}$} & $\begin{array}{ll}50 & 000 \\
12 & 000\end{array}$ \\
\hline
\end{tabular}

Notes: (1) Golfe de Tarente seulement; (2) Côte ionienne de la Calabre uniquement, prise totale mais non mortalité totale; (3) mortalité estimée; (4) illes éoliennes (Lipari) seulement; (5) prise totale dans les eaux siciliennes; (6) mortalité estimée pour toutes les eaux siciliennes; (7) mortalité estimée; (8) prise totale, et non mortalité totale; (9) tortues retenues lorsqu'elles sont capturées; (10) en tenant compte de toutes les prises tunisiennes, plus $15 \%$ de l'estimation du reste des prises méditerranéennes. 
En conjuguant les estimations disponibles pour diverses régions méditerranéennes (résumées au tableau 27), on aboutit à une prise totale de 20.000 à 35.000 tortues au cours des dernières années. La plupart d'entre elles sont des Caretta, et généralement des quasi adultes ou adultes. La plupart sont prises par accident, et une proportion inconnue d'entre elles survivraient à la capture. Si l'on en juge par des estimations préliminaires qui nous viennent de Malte (Gramentz, in litt., 1988), qui suggèrent que de $15 \%$ à $50 \%$ des tortues prises à des aplets peuvent survivre et être relâchées, on peut estimer à 12.000 le nombre total de tortues tuées chaque année (ce chiffre on l'obtient tenant compte de la totalité des prises tunisiennes, entièrement retenues, en ajoutant $15 \%$ du reste des prises méditerranéennes totales).

Selon l'information actuelle, il pourrait y avoir quelque 2.000 Caretta femelles à nicher annuellement en Méditerranée. Si $l^{\prime}$ on suppose un cycle de nidification de deux ou trois ans, et un rapport des sexes à peu près en équilibre, il pourrait y avoir de 8.000 à 12.000 Caretta adultes dans la région. Il est donc théoriquement possible que les prises incidentes enlèvent à la population régionale totale un nombre d'individus plus grand que ne 1 'est le contingent de reproductrices annuel: de 1,5 fois plus grand au minimum, ou au maximum plusieurs fois plus grand, cette situation pouvant exister depuis de nombreuses années. Ces calculs éléinentaires, si les données sur les prises et les nidifications sont suffisamment complètes et fiables, indiqueraient ou bien 1) que la population reproductrice de Caretta de la Méditerranée connaîtrait un rapide déclin quantitatif lorsque les survivantes de l'actuel contingent nicheur atteindraient la fin de leur activité reproductive, ou bien 2) que la population de Caretta quasi adulte et adulte de la Méditerranée est continuellement refournie par des tortues pénétrant dans le bassin (probablement surtout par le détroit de Gibraltar et provenant principalement des plages de ponte nord-américaines). 


\subsection{EXPLOITATION DES TORTUES}

On connaît fort peu l'histoire de l'exploitation des tortues en Méditerranée. La documentation et les témoignages directs se rapportant à la grande prise commerciale de tortues à des fins alimentaires ne datent, dans l'ensemble, que du siècle présent, des milliers de tortues vertes étant capturées en Méditerranée orientale (voir tableau 28). Toute $l^{\prime}$ information disponible suggère que les tortues étaient prises principalement ou totalement en mer, et non capturées sur les plages de ponte; mais la documentation est incomplète.

Il existe une certaine incertitude quant aux espèces qui faisaient l'objet du commerce des tortues au début du siècle. Comme noté à la section 5.4. supra, d'anciens auteurs tels que Lortet (1883, 1887 ) et Gruvel (1931) rapportent que la caouanne, Caretta caretta ( = Thalassochelys caouana ou T. Caretta) était de loin la tortue la plus abondante en Méditerranée orientale. Gruvel (1931) dit que Caretta caretta était l'espèce la plus utilisée par le commerce de tortues en Méditerranée orientale dans les années 20, et Flower (1933) observe l'utilisation très répandue, en Méditerranée, de Caretta, comme élément de base de la "soupe à la tortue". Selon Gruvel (1931), un seul passage de chalut dans le golfe d'Iskendurun pouvait ramener une soixantaine de Caretta, destinées à être exportées en Angleterre via Chypre et Alexandrie.

En revanche, Hornell (1935) et Sella (1982) signalent que Chelonia mydas était pratiquement la seule espèce que prenaient les pêcheurs au large de ce qui est aujourd'hui Israël septentrional, pendant les années 20 et 30 , et dans les golfes d'Iskenderun et de Tarse (Turquie) au cours des années 50 et 60 . Hornell a travaillé en Palestine, tandis que Gruvel explorait la côte syrienne et ce qui est devenu le Liban, et il est concevable que Chelonia ait été alors prédominante dans la première nommée de ces régions, et que Caretta l'ait été dans la seconde. On ne peut cependant exclure la possibilité de fausse identification.

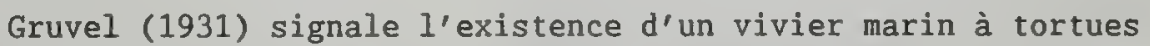
à Fort Kait Bey, près d'Alexandrie (Egypte), d'une profondeur d'environ un mètre, enclos de barres de fer laissant passer les eaux mais empêchant les tortues de s'échapper. Ces dernières étaient amenées de Chypre, d'Iskenderun, de Beyrouth et d'autres ports, et gardées jusqu'à ce qu'un cargo parte pour Londres, où existe un important marché pour les tortues. Hornell (1935) rapporte que des tortues prises dans les eaux palestiniennes étaient exportées vers l'Egypte au cours des années 20, très probablement pour être réexportées vers Londres; les prises de tortues rapportées par Sella (1982), sur la base de renseignements obtenus de vieux pêcheurs opérant au large d'Acre (Israël), alimentaient probablement ce même marché. La Turquie semble avoir été, après la deuxième guerre mondiale, la principale source de C. mydas méditerranéennes pour le commerce; les tortues étaient préparées à Iskenderun et les produits exportés vers l'Europe.

Les grandes prises commerciales et le grand commerce des tortues ont décliné en Méditerranée au cours des deux dernières décennies, probablement en raison de plusieurs facteurs, dont: le développement de la législation protectrice, les restrictions à la commercialisation de la faune (découlant, par exemple, de la Convention sur le commerce international des espèces sauvages de flore et de faune menacées d'extinction), le déclin de la consommation de mets de luxe, tels que la soupe à la tortue, et la diminution des réserves de tortues elles-mêmes. 
Selon l'information actuelle, toujours incomplète, la prise volontaire de tortues est aujourd'hui généralement restreinte à la Tunisie (où l'on prend chaque année jusqu'à 6.000 Caretta à des fins alimentaires), à Malte, à la Sicile et aux îles voisines, ainsi qu'à la Sardaigne (où l'on prend et consomme beaucoup moins de tortues), ainsi qu'à l'Egypte (pour laquelle on ignore la quantité de tortues capturées). Il $n^{\prime} y$ a pas eu confirmation d'une prise intentionnelle de Chelonia (bien que l'espèce puisse faire l'objet de prises en Egypte), et les actuelles populations, de proportions relativement modestes, ne pourraient certainement résister à une exploitation intensive.

Tableau 28. Résumé de l'historique des prises commerciales de tortues en des sites majeurs connus de la Méditerranée.

Données de Sella (1982). Les chiffres représentent des estimations minimales des prises. Par exemple, les données pour Israël ne se rapportent qu'à une entreprise de pêche; on sait que d'autres sont intervenues au cours de la période en cause, mais nous n'avons aucune information à leur sujet. On sait aussi que des tortues sont capturées dans d'autres pays, mais sans connaître le détail.

\begin{tabular}{lll} 
Région & Espèces & Nombre/an Prise totale \\
\hline
\end{tabular}

Israël $\quad$ C.mydas $\quad \begin{array}{r}\text { années } 20- \\ \text { début des } \\ \text { années } 30\end{array}$

Turquie $\quad \underline{\text { C.mydas }}$ Début des

années 50

- années 60 environ $2.000>30.000$

Dans l'ensemble, l'exploitation volontaire ne semble pas constituer une menace majeure pour les tortues de Méditerranée, mais on peut s'inquiéter sérieusement de l'ampleur des prises de Caretta que l'on signale pour les eaux tunisiennes. Des tortues baguées en Italie et à Zante sont recapturées en Tunisie et, comme les retours de bagues ne représentent toujours qu'une très faible proportion du nombre d'animaux bagués, il est raisonnable de supposer que cette pêche est cause de forte mortalité parmi les populations de Caretta de la Méditerranée centrale. Conjuguée à d'autres facteurs adverses dont souffrent ces populations, particulièrement aux sévères perturbations des plages de ponte de Zante, une telle mortalité provoquée deviendra sans doute intolérable à long terme.

Il importe donc de réduire les prises à un niveau compatible avec la survie à longue échéance des populations; compte tenu du piètre état actuel de conservation des tortues de la Méditerranée, une prise quasi nulle est souhaitable. Néanmoins, comme Lescure (comm. pers.) l'a souligné, les prises de tortues sont aujourd'hui, en Tunisie, d'une grande importance socio-économique; la chair de tortue est meilleur marché que celle du poisson et constitue une source d'alimentation accessible aux collectivités côtières. 


\subsection{POLLUTION}

On sait peu de choses sur les polluants marins et leur impact sur les tortues en mer et la plupart des études sont très récentes. Dans les eaux maltaises, sur 99 Caretta examinées, 20 se sont révélées contaminées, dont 17 d'entre elles ayant du pétrole brut dans la cavité buccale et l'intestin et quelques-unes des objets en plastique ou en métal (Gramentz, 1988). Bien qu'on ne l'ait pas encore évaluée avec précision, la pollution par le pétrole est probablement un problème quasi général dans le bassin méditerranéen.

Certains détritus rejetés par les navires échouent certainement sur les plages fréquentées par les tortues, notamment à Chypre et à l'Est de la Turquie, mais leurs effets sur les femelles reproductrices et les nouveaux-nés ne sont pas très nets. Les débris de plastique en mer risquent de poser un problème supplémentaire; on a observé, en effet, que les Caretta et Dermochelys avalent des détritus en plastique (qui peuvent flotter dans l'eau à la manière des coelentérés pélagiques qui constituent une part importánte de l'alimentation des deux espèces) (Van Nierop et Den Hartog, 1984). Ce phénomène n'est pas étranger à la mort de certains spécimens.

Dans le sud de l'Italie et le sud-est de la Turquie, les résidus des métaux lourds et de l'agrochimie sont supposés polluer les eaux où vivent des tortues (notamment la principale zone de nidification de la tortue verte en Méditerrannée). Il faut faire des études plus approfondies sur ces zones en particulier et sur le phénomène en général; bien que moins ouvertement nuisible que les prises accidentelles ou l'aménagement des plages, la pollution est potentiellement une menace tout aussi grande pour les populations de tortues. 


\section{BSPECES NON reproductriceS ET OCCASIONNELLES}

\subsection{OBSERVATIONS GENRRALES}

La présente section intéresse quatre espèces, dont $I^{\prime}$ une seulement, la tortue-cuir Dermochelys coriacea, est signalée régulièrement (quoique rarement) en Méditerranée. Il est possible que quelques couvées de Dermochelys soient déposées de temps à autre sur des plages méditerranéennes, mais, dans $l^{\prime}$ hypothèse où il n'y aurait aucun nidification régulier, le nombre assez important de tortues à cuir signalées dans le bassin doit représenter une immigration ayant son origine dans les colonies reproductrices de l'Atlantique.

Deux autres espèces, la tortue à écaille (Eretmochelys imbricata) et Lepidochelys kempii, sont connues en Méditerranée, mais on présume qu'il ne s'agit que d'immigrantes accidentelles. Sous l'angle documentaire, ces tortues sont représentées respectivement par trois et par un spécimens de musée. Une quatrième espèce, Lepidochelys olivacea, est peut-être aperçue très rarement, mais nous n'avons aucun document relatif à des spécimens ayant leur origine en Méditerranée.

\subsection{LA TORTUE-CUIR (DERMOCHELYS CORIACEA)}

Une étude récente (Lescure et coll., sous presse) conclut que, si Dermochelys est connue au moins depuis la fin du XVIIIe siècle comme faisant partie de la faune marine de la Méditerranée, ses nidifications ont toujours été extrêmement rares.

Un petit nombre de tortues-cuir femelles ont été capturées en Méditerranée et disséquées; on a trouvé des ovules bien développés dans celles qui avaient été prises au printemps, et des ovaires réduits dans les animaux capturés à l'automne. Comme l'ont écrit Lescure et coll., ce fait inciterait à supposer que la tortue-cuir pondrait dans le bassin méditerranéen. Bien que l'on ait signalé des émergences ou des nidifications apparents en Israël, en Italie et à Malte, nous ne connaissons aucun témoignage bien documenté du nidification de Dermochelys en Méditerranée.

Delaugerre (1988) cite une publication de 1858 qui signalait un nidification de tortue à cuir sur la côte barbaresque du Maroc; mais il note qu'on ne peut aujourd'hui contrôler cette indication. Selon Sella (1982), si Dermochelys est plutôt rare en Israël, on y a trouvé des signes de nidification possible; mais les seules données citées à l'appui se rapportent à des empreintes aperçues en juin 1963 sur une plage de Palmachim, au sud de Tel Aviv. On a attribué les empreintes à cette espèce en raison de leur largeur $(1,10 \mathrm{~m})$, mais ce $n^{\prime}$ est pas concluant, et $I^{\prime}$ on n'a trouvé aucun nid. Bruno (1978) signale des nidifications apparents de Dermochelys, à la suite de la découverte de deux jeunes sur la plage de Macconi (province de Gela), sur la côte méridionale de Sicile, en septembre 1961, ainsi que d'veufs dans le même secteur en juin 1967; d'autres observateurs ont exprimé des réserves quant à des témoignages dispersés et contradictoires, et ne sont nullement convaincus que des pontes ont eu lieu (Argano, 1979; Lescure et coll., sous presse). Plus récemment, Gramentz (in litt., 26 juillet 1988) a signalé une émergence de tortues-cuir à Lampedusa (Italie), qui n'a pas été suivie de nidification réussi. 
La meilleure preuve que des nidifications accomplis peuvent avoir eu lieu en Méditerranée est constituée par la capture occasionnelle de jeunes et très jeunes spécimens. Delaugerre (1988) estime qu'un très jeune animal (longueur de carapace $73 \mathrm{~mm}$ ), conservé depuis 1835 au Muséum national d'histoire naturelle de Paris (MNHNP 632), et que l'on dit d'origine méditerranéenne, constitue le seul témoignage que Dermochelys ait niché dans le bassin, ce qui ne peut être considéré comme concluant, vu les fréquentes erreurs de localisation dont sont l'objet les spécimens de musée aussi anciens. Un deuxième spécimen, qui se trouve aujourd'hui au musée de Florence (MZUF 12142), a été trouvé près de Messine, en Sicile, en avril 1896, et il a quelque $66 \mathrm{~mm}$ de longueur de carapace. Ces deux spécimens ont environ $10 \mathrm{~mm}$ de plus que n'en ont les nouveau-nés (de la population de Guyane française), et pourraient avoir moins d'un an d'âge. Au moins deux autres jeunes Dermochelys auraient été capturées sur les côtes de Sicile (Bruno, 1978), mais nous n'avons ni détails ni spécimen.

En résumé, on n'a jamais vu de Dermochelys femelle émerger pour nicher, ou nidifiant effectivement, sur une plage méditerranéenne, et il n'existe aucune documentation valable d'émergence cu de nidification. Bien qu'il semble tout à fait possible, et même probable, qu'il y a quelques nids chaque année sur les côtes méditerranéennes, il n'y a sans doute pas de plages de ponte régulières, et aucun témoignage ne suggère que Dermochelys ait jamais niché en Méditerranée, du moins en nombre important.

On a cependant signalé souvent la tortue à cuir en mer, principalement dans le centre et l'ouest de la Méditerranée, bien que les témoignages (résumés par Delaugerre, 1988) existent pour à peu près tous les pays du pourtour méditerranéen, à l'exception apparemment de l'Albanie et du Maroc. Il s'agit ici, de manière générale, d'une espèce essentiellement pélagique qui affectionne les eaux ouvertes.

On signale généralement des animaux isolés, mais de petits groupes ont été observés au voisinage du détroit de Gibraltar, où 24 individus (par troupes de 2 à 11) ont été vus en moins de quatre ans (Delaugerre, 1988). Fernandez et Moreno (1984) indiquent avoir vu 11 Dermochelys mortes sur des plages de Ceuta (enclave espagnule sur la côte marocaine, du côté sud du détroit) en novembre-décembre 1980, et un individu chaoue fois, en juillet 1982 et août 1983. La zone du détroit de Gibraltar présente la plus forte densité de signalements de tortues-cuir du bassin méditerranéen, probablement surtout en raison du fait que les individus ou les groupes qui se déplacent à travers ce bras de mer relativement étroit sont nécessairement moins éparpillés et plus aisément remarqués.

Néanmoins, comme c'est le cas pour toutes les tortues vues en mer, la distribution des signalements reflète autant celle des observateurs que celles des tortues, et des groupes semblables pourraient se trouver ailleurs en Méditerranée. Selon Delaugerre (1988), les tortues en question pénètrent en Méditerranée en été et retournent dans l'Atlantique en hiver, et elles proviennent sans doute de vastes colonies reproductrices de Guyane française. Aucun passage par le détroit n'a encore été démontré par baguage ou par d'autres moyens. 


\subsection{LA TORTUE A ECAILLE ERETHOCHELYS IMBRICATA}

Bien qu'on dise souvent que cette espèce se rencontre en Méditerranée, on n'a jamais signalé de nidification, et très peu de présence en mer (la plupart des mentions de l'espèce, dans la documentation, répètent simplement des mentions antérieures qui ne sont pas elles-mêmes bien fondées). Trois spécimens de musée d'Eretmochelys, dont un au moins semble avoir une provenance méditerranéenne relativement sure, constituent les seules preuves apparemment tangibles de l'existence de l'espèce dans le bassin. Un spécimen de jeune femelle (récemment redécouvert au Musée d'histoire naturelle de Marseille) a été capturé près de cette ville en 1909 (Fretey, 1987; Delaugerre, 1988). Gramentz (in litt, 26 juillet 1988) rapporte qu'on a également pris Eretmochelys près des côtes méridionales de la Sicile et à Malte, et que ces prises sont aujourd'hui respectivement représentées par des spécimens situés à Palerme et au Musée d'histoire naturelle, Mdina, à Malte. En outre, Gruvel (1931) semble avoir vu personnellement Eretmochelys en Méditerranée orientale.

La tortue à écaille ne peut être considérée, en Méditerranée, que comme une espèce accidentelle et occasionnelle, et les spécimens qui pourraient exister dans le bassin y pénètrent probablement par le détroit de Gibraltar ou le canal de Suez.

\subsection{LEPIDOCHELYS REMPII}

Cette espèce, comme la tortue à écaille, ne doit être considérée que comme accidentelle et occasionnelle en Méditerranée. Comme la seule plage de ponte connue de cette espèce très menacée se trouve dans le golfe du Mexique, et que l'on sait qu'un nombre appréciable d'individus se déplacent vers le nord et l'est en suivant les courants de l'Atlantique septentrionale, il semble certain que si des individus pénètrent en Méditerranée, ils doivent le faire par le détroit de Gibraltar. Le seul témoignage documenté de la présence de l'espèce en Méditerranée est un specimen conservé au Musée d'histoire naturelle de Mdina, à Malte, et qui a initialement été capturé à environ un mille marin de l'entrée du grand port de La Valette, en octobre 1929 (Brongersma et Carr, 1983).

\subsection{LEPIDOCHELYS OLIVACEA}

Le seul indice de l'existence de cette espèce en Méditerranée est la constatation, par Mertens (1959), de la présence d'un individu dans l'Aquarium de Rhodes (Grèce), que cet auteur a identifié comme étant L. olivacea. Comme l'ont fait observer Brongersma et Carr (1983), il est possible que la tortue ait été importée de l'extérieur de la Méditerranée, et il $n^{\prime} y$ a jamais eu de signalement bien établi de l'espèce dans le bassin. 
7.

Le texte et le tableau qui suivent résument les principaux protocoles et conventions internationaux contenant des dispositions en vue de la protection des tortues marines de la Méditerranée.

Il importe de noter que l'efficacité de tels accords dépend a. de l'efficacité de la législation nationale adoptée pour permettre au pays qui les a ratifiés de remplir ses obligations; b. de la rigueur avec laquelle ladite législation est appliquée. Le droit national n'a pas toujours été formulé au moment de la ratification, et l'application laisse souvent à désirer.

Les plages de ponte situées dans les Etats parties à la Convention de Berne ont été largement aménagées au détriment des populations de tortues; celles-ci sont capturées en grand nombre dans certains pays où les prises sont théoriquement prohibitées par la législation nationale et contreviennent à la Convention sur le commerce international des espèces de faune et de flors sauvages menacées d'extinction (voir infra), qui interdit l'importation, de la région méditerranéenne, d'espèces désignées à l'annexe I; nous n'avons pas d'exemple d'efforts internationaux de gestion des espèces au cours de la phase migratoire. Il apparait donc clairement que les accords internationaux existants pour la protection des tortues sont violés par plusieurs pays et ne sont certainement pas bien utilisés pour améliorer la préservation des tortues marines de la Méditerranée.

Tableau 29. Principaux accords internationaux intéressant les tortues marines de la Méditerranée, et Etats signataires.

Clef: * = Etat partie (dans quelques cas, l'Etat a signé l'accord mais ne l'a pas encore ratifié: il n'est pas alors considéré comme partie). Sources des données: $\mathrm{a}=$ de Klemm (1989), $\mathrm{b}=$ WCMC (Centre mondial de contrôle de la conservation), $c=$ Alliance pour la nature/PNUE (1988).

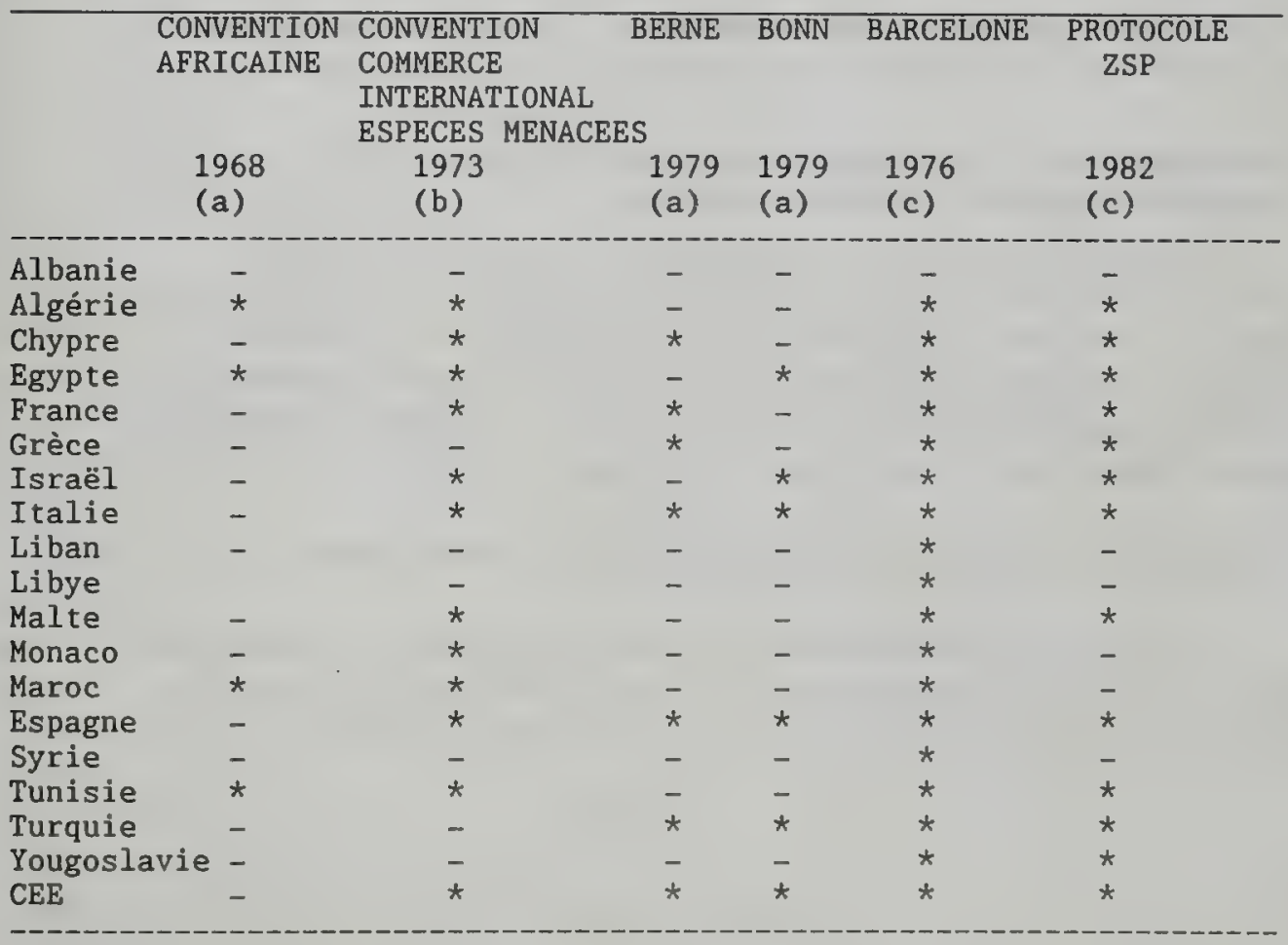




\section{1) Convention africaine sur la conservation de la nature et} des ressources naturelles

Toutes les tortues marines figurent dans la catégorie A de I'annexe à cette Convention, et doivent ainsi faire l'objet d'une protection totale. Le commerce national ou international des espèces protégées est interdit, sauf possession d'un permis.

2) Convention sur le commerce international des espèces de faune et de flore sauvages menacées d'extinction

Toutes les tortues marines figurent à l'annexe I de cette Convention. Cette annexe énumère les espèces menacées d'extinction et qui sont ou pourraient être touchées par le négoce. Le commerce international est soumis à une règlementation particulièrement stricte ayant pour but d'assurer leur survie, et il ne doit être autorisé que dans des cas exceptionnels. L'importation de spécimens de la Méditerranée est également interdite en $1^{\prime}$ 'absence d'un permis préalable, dont la délivrance est étroitement règlementée. En outre, tous les Etats membres de la CEE non séparément parties à cette convention doivent se conformer au Règlement CEE $3626 / 82$, qui rend la Convention applicable au sein de la Communauté.

3) Convention relative à la conservation de la vie sauvage et des habitats naturels de l'Burope (Convention de Berne)

Toutes les tortues marines de la Méditerranée figurent à l'annexe II. L'article 6 exige que chaque partie contractante prenne les mesures législatives et administratives appropriées et nécessaires pour assurer une protection spéciale de telles espèces. La Convention interdit: la capture délibérée, la garde et l'abattage, la destruction ou l'endommagement délibérés des sites de reproduction ou de nidification, la destruction ou l'enlèvement délibérés des oeufs, la perturbation, la possession et le commerce lorsqu'ils sont contraires aux buts de la Convention. L'article 10 impose aux parties de coordonner leurs efforts pour la protection des espèces migratoires énumérées à l'annexe II (et à l'annexe III), passant ou séjournant sur leur territoire.

4) Convention sur la protection des espèces migratoires d'animaux sauvages (Convention de Bonn)

Toutes les tortues marines de Méditerranée y figurent à $I^{\prime}$ annexe $I$, en tant qu'espèces migratoires menacées d'extinction. Les parties ont l'obligation d'assurer à ces espèces une protection stricte. Toutes les tortues marines de la Méditerranée figurent également à l'annexe II. Les parties doivent s'efforcer de conclure des accords pour la protection des espèces migratoires qui passent ou séjournent dans leurs limites territoriales.

5) Convention pour la protection de la Méditerranée contre la pollution (Convention de Barcelone)

La Convention de Barcelone oblige les parties contractantes à prendre toutes les mesures appropriées pour réduire ou prévenir la pollution et protéger le milieu marin. Adoption: 18 février 1976; entrée en vigueur: 12 février 1978. 
6) Protocole sur les zones spécialement protégées (ZSP) de la Méditerranée

Le dernier des trois protocoles élaborés dans le cadre de la Convention de Barcelone. Son objet est la protection des espèces menacées de Méditerranée et des zones importantes pour leur survie. Adopté à Genève, le 2 avril 1989. Le phoque moine de la Méditerranée, Monachus monachus, et les tortues marines ont occupé ou occupent un rang tout à fait prioritaire en vue de l'action menée ou à mener au cours de la période 1985-95 (Déclaration de Gênes, 9-13 septembre 1985). On a préparé, vers la fin 1989, un "plan d'action pour la préservation des tortues marines de la Méditerranée" (Anon. e, 1989). 


\section{REMERCIEMENTS}

Plusieurs personnes ont très aimablement consacré une partie de leur temps et de leur énergie pour aider à la préparation du présent rapport, en fournissant des copies, des témoignages et renseignements inédits, en répondant à des questionnaires (ou en transmettant des données de ce genre, dans le passé, au Centre de contrôle de la protection de l'UICN). Tout l'intérêt que représentait ce rapport est le fruit de leur coopération et l'auteur exprime sa sincère gratitude à tous ceux qui lui sont venus en aide de cette manière, dont:

M. Amina, R. Argano, H. Baccar, S. Balzan, A.E. Baldacchino,

V. van den Berk, L.D. Brongersma, L.F. Cassar, A. Chalabi, A.E. Chircop,

M. Cocco, K. Corbett, M. Delaugerre, A. Demetropoulos, J. Fretey,

R. Geldiay, D. Gramentz, M. Hadjichristophorou, R. Jesu, M. Kasparek,

H.H. Kouyoumjian, F. Koyuncuoglu, Z. Kuller, E. Lanfranco, L. Laurent, J. Lescure, D. Margaritoulis, J. Mayol, H. Mendelssohn, C. Mutlu, M.G. di Palma, S. Pisanty, R. Sagarminaga, J. Sultana, A. Taha,

L. Venizelos, C. Whitmore.

Des observations écrites sur la première version du présent rapport ont été reçues de $V$. van den Berk, $K$. Corbett,

M. Hadjichristophorou, C. de Klemm, D. Margaritoulis, J. Maigret, J. Robbins, B. Rolle et J. Vournas. Des commentaires oraux de plusieurs autres sources ont été formulés lors de la deuxième réunion du groupe d'experts des tortues marines, qui s'est tenue au Conseil de 1'Europe en octobre 1989. Les observations relatives aux faits et aux interprétations ont été incorporées, dans toute la mesure du possible, dans le présent texte, et l'auteur exprime sa reconnaissance à tous les intéressés.

Cette version définitive contient une information nouvelle se rapportant principalement à l'Afrique du Nord, à la Grèce et à la Turquie. Je dois des remerciements particuliers à Luc Laurent, qui m'a transmis des exemplaires de rapports relatifs à son travail sur le terrain au Maroc, en Algérie et en Tunisie; à Dimitri Margaritoulis et $\mathrm{T}$. Arapis, qui $\mathrm{m}^{\prime}$ ont fourni des rapports sur des travaux effectués en Grèce par la Société de protection des tortues marines; et à Miriam Langeveld, pour des exemplaires de son rapport (avec G. Sarigül) d'un travail sur le terrain dans le sud-est de la Turquie. Beaucoup de nouveaux renseignements sur la Turquie ont été tirés du rapport de I. Baran et $M$. Kasparek, qui présentent les résultats de l'enquête de plage menée en 1988 par le Fonds mondial pour la nature, la CEE et le DHKD.

Je dois aussi de grands remerciements à Veronica Greenwood pour son méticuleux travail d'édition et pour la production d'un exemplaire photocopiable. 


\section{REFERENCES}

Anon. a. (1987). "On the spot appraisal" of Caretta caretta conservation at Laganas Bay, Zakynthos, Greece. 25-27 June 1987. Document T-PVS (87) 19, presented at 1st meeting of Group of Experts on Caretta caretta and Chelonia mydas (Bern Convention), 1-2 September, Strasbourg.

Anon. b. (Corbett, K.). (1987). "On the spot appraisal" of Caretta caretta conservation at Laganas Bay, Zakynthos, Greece. 25-27 June 1987. Document T-PVS (87) 18, presented at 1st meeting of Group of Experts on Caretta caretta and Chelonia mydas (Bern Convention), 1-2 September, Strasbourg.

Anon. c. (Corbett, K., Koester, V. [Chairman of the Standing Committee of the Bern Convention] and Ribaut, J.P. [Head of the Environment Conservation and Management Division, Council of Europe] (1989). On the spot appraisal of Caretta caretta turtle conservation at Zakynthos (Greece). Document T-PVS (89) 37.

Anon. d. (Woods, R., Ed.). (1989). 1988, 1989, summary of Annual Reports. Marine Turtle Project Update. No. 1. Pp. 1-4 (Newsletter).

Anon. e. (1989). Report of the Sixth Ordinary Meeting of the Contracting Parties to the Convetnion for the Protection of the Mediterranean Sea against Pollution and its related Protocols. Athens, 3-6 0ctober 1989. UNEP(OCA)/MED IG. 1/5/ 1 November 1989.

Argano, R. (1979). Preliminary report on Western Mediterranean Sea Turtles. Unpublished annual report on WWF Project No. 1474.

Argano, R., and Baldari, F. (1983). Status of Western Mediterranean Sea Turtles. Rapp. Comm. int. Mer Médit., 28(5): 233-235. (not seen, cited by Lescure in litt.).

Ashkenazi, S., and Sofner, A. (1988). Conservation of the endangered sea turtles Chelonia mydas and Caretta caretta in Israel. Rapports et Procès-Verbaux des Réunions. Condensés des travaux présentés lors du XXXI Congrès-Assemblée Plénière, Athènes (Grèce) Volume 31 (2): 286, V-III6. C.I.E.S.M., Monaco.

Baran, I. (1987). Amphibians and Reptiles. In, Anon., Biological Diversity in Turkey. Environmental Problems Foundation of Turkey, Ankara. Pp. 163-175.

Baran, I., and Kasparek, M. (1988). Study on the nesting sites and population status of the Loggerhead Turtle and the Green Turtle in Turkey. Unpublished interim report to WWF.

Baran, I. and Kasparek, M. (1989a). Marine Turtles - Turkey. Status survey 1988 and recommendations for conservation and management. World Wide Fund for Nature.

Baran, I. and Kasparek, M. (1989b). On the whereabouts of immature sea turtles (Caretta caretta and Chelonia mydas) in the eastern Mediterranean. Zoology of the Middle East 3: 31-36.

Bjorndal, K. (Ed.) (1982). Biology and Conservation of Sea Turtles,

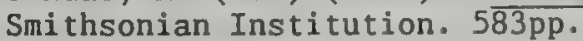


Brongersma, L.D. (1982). Marine turtles of the Eastern Atlantic Ocean. In Bjorndal, K. (Ed.). Biology and Conservation of Sea Turtles, Smithsonian Institution, pp. 407-416.

Brongersma, L.D., and Carr, A.F. (1983). Lepidochelys kempii (Garman) from Malta. Proceedings Koninklijke Nederlandse Akademie van Wetenschappen, Series C, 86(4): 445-454.

Bruno, S. (1973). Problemi di conservazione nell campo dell' erpetologica. Atti 3rd Simp. naz. Conserv. Nat. Bari, 2: 117-226.

Bruno, S. (1978). Le tartarughe nei mari italiani e nel Mediterraneao. Natura e montagna, Bologna, 25(3): 5-17.

Caminas, J.A. (1988). Incidental captures of Caretta caretta (L.) with surface long-lines in the Western Mediterranean. Rapports et Procès-Verbaux des Réunions. Condensés des travaux présentés lors du XXXI Congrès-Assemblée Plénière, Athènes (Grèce) Volume $31(2): 285$, V-III4. C.I.E.S.M., Monaco.

Capocaccio, L. (1966). Variabilità della popolazione mediterranea di Caretta caretta (L.) (Testudines). Annali del Museo Civico di Storia Naturale di Genova, 76: 1-22.

Carr, A. (1986). Rips, FADS, and Little Loggerheads. Bioscience $36(2): 92-100$.

Carr, A. (1987). New Perspectives on the Pelagic Stage of Sea Turtle Development. Conservation Biology 1(2): 103-121.

Dean, J.M., and Talbert, 0.R. (1975). The Loggerhead turtles of Kiawah Island. Environmental Inventory of Kiawah Island, pp.T-1 to T-19. Colombia, South Carolina, Environmental Research Centre (cited by Shabica, not seen).

Delaugerre, M. (1988 [1987]). Statut des tortues marines de la Corse (et de la Méditerranée). Vie Milieu 37(3/4): 243-264.

De Metrio, G., and Megalofonou, P. (1988). Mortality of marine turtles (Caretta caretta L. and Dermochelys coriacea L.) consequent to accidental capture in the Gulf of Taranto. Rapports et ProcèsVerbaux des Réunions. Condensés des travaux présentés lors du XXXI Congrès-Assemblèe Plénière, Athènes (Grèce) Volume $31(2): 285$, V-III5. C.I.E.S.M., Monaco.

De Metrio, G., Petrosino, G., Matarrese, A., Tursi, A., and Montanaro, C. (1983). Importance of the fishery activities with drift lines on the populations of Caretta caretta (L.) and Dermochelys coriacea (L.) (Reptilia, Testudines), in the Gulf of Taranto. 0ebalia N.S., 9:43-53.

Demetropoulos, A., and Hadjichristophorou, A. (1986). In litt., 28 June.

Demetropoulos, A., and Hadjichristophorou, A. (1987). Turtles and Turtle Conservation in Cyprus (handout for visitors to Lara Turtle Station). Department of Fisheries, Nicosia.

Deraniyagala, P.E.P. (1951). Mass movement in some marine turtles and cuttle fish. Spolia Zeylan., Bull. Nat. Mus. Ceylon, 26:1,17. 
Despott, G. (1915). The reptiles of the Maltese Islands. The Zoologist 19 (No. 891): 821-827.

Doumergue, M. (1899). Essai sur la faune erpétologique de l'Oranie. Bull. soc. géogr. Archéol. Oran. 19-21, 404 pp, 27pl. (not seen, cited by Laurent, 1989a).

Dumont, M. (1973). Le point sur les Tortues marines. Bêtes et Nature 108: 34-36 (not seen, cited by Delaugerre).

Dumont, M. (1974). Les Chéloniens de France. Le courrier de la nature 33: $224-227$.

Ehrhart, L.M., and Witherington, B.E. (1987). Human and natural causes of marine turtle nest and hatchling mortality and their relationship to hatchling production on an important Florida nesting beach. Florida Game and Fresh Water Fish Commission, Nongame Wildlife Programme. Technical Report $N^{0} 1$.

Estrada, M., Vives, F., and Alcaraz, M. (1985). Life and the productivity of the open sea. Chapter 6, pp. 148-197, in Margalef, $M$. (Ed.) Western Mediterranean. Key Environment Series, Pergamon Press Ltd.

Fernandez, P.G., and Moreno, S.C. (1984). Embarrancamiento masivo de ejemplares de Tortuga Laud (Dermochelys coriacea L.) en las costas de Ceuta (Espana, Norte de Africa). Acta Vertebrata, Doñana 11(2): 312-320.

Flower, S.S. (1933). Notes on the recent reptiles and amphibians of Egypt, with a list of the species recorded from that Kingdom. Proceedings of the Zoological Society of London 1933: 735-851.

Fournet, J. (1853). Recherches sur la distribution et sur les modifications des caractères de quelques animaux aquatiques du bassin du Rhône. Ann. soc. Agric. Lyon 2(5): 1-120. (not seen, cited by Laurent, $1989 \mathrm{a})$.

Fretey, J. (1987). Les Tortues. Fascicule 44 in Beaufort, F. de (Ed.) Livre Rouge des Espèces Menacées en France, tome 2, Espèces Marines et Littorales Menacées. Secrétariat de la Faune et de la Flore, Muséum National d'Histoire Naturelle, Paris.

Frommhold, E. (1962). Herpetologische studien in Albanien. Aquarien Terrarien 10(?): 365-370.

Geldiay, R. (1984). Türkiye'nin ege ve akdeniz kiyilarinda yasayan deniz kaplumbagalarinin (Caretta c. caretta L. ve Chelonia m. mydas L.) populasyonlari ve korunmasi ile ilgili arastirmalar. Doga Bilim Dergisi, Ser. A, Cilt 8, Sayi 1: 66-75.

Geldiay, R., Koray, T., and Balik, S. (1982). Status of sea turtle populations (Caretta $c$. caretta and Chelonia $\mathrm{m}$. mydas) in the northern Mediterranean Sea, Turkey. In Bjorndal, K. (Ed.), Biology and Conservation of Sea Turtles, Smithsonian Institution, pp. 425-434.

Gramentz, D. (1986a). Loggerhead Turtles at Lampedusa, Italy. Marine Turtle Newsletter 36:3. 
Gramentz, D. (1986b). Cases of contamination of sea turtles with hydrocarbons. U.N. ROCC INFO N ${ }^{\circ}$ 17: 1-3. Regional 0il Combating Centre, Manoel Island, Malta.

Gramentz, D. (1988). Involvement of Loggerhead Turtle with the plastic, metal, and hydrocarbon pollution in the Central

Mediterranean. Marine Pollution Bulletin 19(1): 11-13.

Groombridge, B. (1982). The IUCN Amphibia-Reptilia Red Data Book. Part 1, Testudines, Crocodylia, Rhynchocephalia. IUCN, Gland, Switzerland and Cambridge, UK.

Groombridge, B. and Luxmoore, R. (1989). The Green Turtle and Hawksbill (Reptilia: Cheloniidae): world status, exploitation and trade. Secretariat of the Convention on International Trade in Endangered Species of Wild Fauna and Flora, Lausanne. Pp. i-vi, $1-601$.

Groombridge, B. and Whitmore, C. (1989). Marine turtle survey in Northern Cyprus. Marine Turtle Newsletter 47: 5-8.

Gruvel, A. (1931). Les Etats de Syrie. (Richesses marines et fluviales. Exploitation actuelle - Avenir). Bibliothèque de la Faune des Colonies Françaises III. Paris, Société d'Editions Géographiques, Maritimes et Coloniales.

Henwood, T.A. (1987). Movements and seasonal changes in Loggerhead Turtle Caretta caretta aggregations in the vicinity of Cape Canaveral, Florida (1978-84). Biological Conservation 40: 191-202.

Hornell, J. (1935). Report on the Fisheries of Palestine. Published on behalf of the Government of Palestine by the Crown Agents for the Colonies, Millbank, London.

Hsü, K.J. (1972). When the Mediterranean dried up. Scientific American, December 1972.

IUCN (1988). 1988 IUCN Red List of Threatened Animals. IUCN, Gland, Switzerland and Cambridge, UK.

IUCN/UNEP (1988). The Mediterranean Monk Seal (Reijnders, P.J.H. et al., Eds.). IUCN, Gland, Switzerland.

Klemm, C. de (1989). Comments on the report to the Council of Europe on Marine Turtles in the Mediterranean (Legal Aspects). Paper T-PVS (89) 20 circulated at 2nd meeting of Group of Experts on Marine Turtles, Strasbourg, 2-3 0ctober 1989.

Kuller, Z. (1986). In litt., 23 November.

Langeveld, M.J. and Sarigül, G. (1989). Sea turtles nesting on beaches of south-east Turkey. A preliminary report with emphasis on the protection of the nesting beaches. Society for the Advancement of Oceanography (SBNO), Amsterdam.

Lanteri, A. (1982). Note sur un important rassemblement de Caretta caretta (Reptilia, Testudines) au large de la Côte Oranaise. Bull. Soc. Herp. Fr., 23: 63-65. 
Laurent, L. (1989a). Les tortues marines en Algérie et au Maroc (Méditerranée). Rapport de mission. Centre d'Activités Régionales pour les Aires Spécialement Protégées. Greenpeace Méditerranée. 48

Laurent, L. (1989b). Report on the status of the Mediterranean Marine Turtle. Presented at Expert Meeting on the Conservation of Mediterranean Marine Turtles, Nicosia, 4-6 July 1989. RAC/SPA-UNPE/MEDU WG. $1 / 3$.

Laurent, L. (in press). Observations pélagiques de la Caouanne, Caretta caretta (Chelonii, Cheloniidae) en Méditerranée occidentale. Bulletin de la Société Herpetologique de France.

Laurent, L., Nouira, S., Jeudy de Grissac, A. and Bradai, M.N. (in press). Les Tortues Marines de Tunisie; premières données. Bulletin de la Société Herpétologique de Franc.

Lescure, J., Delaugerre, M., and Laurent, L. (in press). La nidification de la Tortue Luth, Dermochelys coriacea (Vandelli, 1761) en Méditerranée. Mésogee.

Lortet, L. (1883). Etudes Zoologiques sur la Faune du Lac de Tibériade Suivies d'un Aperçu sur la Faune des Lacs d'Antioche et de Homs. I. Poissons et Reptiles du Lac de Tibériade et de quelques autres Parties de la Syrie. Archives du Muséum d'Histoire Naturelle de Lyon, III.

Lortet, L. (1887). Observations sur les Tortues Terrestres et Paludines du Bassin de la Méditerranée. Archives du Muséum d'Histoire Naturelle de Lyon, IV.

Loveridge, A., and Williams, E.E. (1957). Revision of the African tortoises and turtles of the suborder Cryptodira. Bulletin of the Museum of Comparative Zoology, Harvard 115(6): 163-557.

Maldonado, A. (1985). Evolution of the Mediterranean basins and a detailed reconstruction of the Cenozoic paleoceanography. In: Margalef, M. (Ed.), Western Mediterranean. Key Environments Series, Pergamon Press Ltd. Chapter 2, pp. 17-59.

Mann, T.M. (1977). Impact of developed coastline on nesting and hatchling sea turtles in southeastern Florida. Master's Thesis, Florida Atlantic University.

Manzella, S.A., Fontaine, C.T., and Schroeder, B.A. (1988). Loggerhead sea turtle travels from Padre Island, Texas to the mouth of the Adriatic Sea. Marine Turtle Newsletter 42: 7.

Margaritoulis, D. (1980). Nesting of the sea turtles Caretta caretta on Zakynthos Island. Nature: Bulletin of the Hellenic Society for the Protection of Nature. Athens, No. 22: 41-43.

Margaritoulis, D. (1982). Observations on Loggerhead Sea Turtle Caretta caretta activity during three nesting seasons (1977-1979) in Zakynthos, Greece. Biological Conservation 24: 193-204.

Margaritoulis, D. (1986). Captures and strandings of the Leatherback Sea Turtle, Dermochelys coriacea, in Greece (1982-1984). Journal of Herpetology 20(3): 471-474. 
Margaritoulis, D. (1987a). Nesting activity and factors affecting breeding of the Loggerhead Sea Turtle Caretta caretta (L.) in Greece. Ministry of the Environment, Physical Planning and Public Works, Athens. Final Report on contract No. ENV-790-GR.

Margaritoulis, D. (1987b). Monitoring of Loggerhead Sea Turtle nesting in the Bay of Kiparissia, Greece. Sea Turtle Protection Society, Athens. Pp. 1-12, plus figures.

Margaritoulis, D. (1988a). Post-nesting movements of Loggerhead Sea Turtles tagged in Greece. Rapports et Procès-Verbaux des Réunions. Condensés des travaux présentés lors du XXXI Congrès-Assemblée Plénière, Athènes (Grèce) Volume 31 (2): 284, V-III3. C.I.E.S.M., Monaco.

Margaritoulis, D. (1988b). Nesting of the Loggerhead Sea Turtle Caretta caretta on the shores of Kiparissia bay, Greece, in 1987. Mésogée 48: 59-65.

Margaritoulis, D., Arapis, T., Kornaraki, E., and Mytilineou, C. (1986). Three specimens of the Green Sea Turtle Chelonia mydas (L.) recorded in Greece. Biologia Gallo-hellenica 12: 237-243.

Marinos, P. (1981). On the distribution of sea turtles in Greek waters. Marine Turtle Newsletter 19: 13-14.

Marinos, P. (1982). Final Report. WWF/IUCN Project No. 1822. Greece, Sea Turtle Conservation, Island of Zakynthos.

Mayol, J., and Castello Mas, M. (1983). Contribución al conocimiento de la Tortuga Boba en las Baleares. Ministerio de Agricultura, Pesca y Alimentación. ICONA, Palma de Mallorca.

Mertens, R. (1959). Zur kenntnis der lacerten auf der Insel Rhodos. Senckenbergiana biologica $40(1 / 2): 15-24$.

Mortimer, J.A. (1982). Factors influencing beach selection by nesting sea turtles. In Bjorndal, K. (Ed.). Biology and Conservation of Sea Turtles, Smithsonian Institution, pp. 45-51.

Mrosovsky, N. (1983). Conserving Sea Turtles. The British Herpetological Society, London. $176 \mathrm{pp}$.

Ogren, L., and McVea, C. (1982). Apparent hibernation by sea turtles in North American waters. In Bjorndal, K. (Ed.). Biology and Conservation of Sea Turtles, Smithsonian Institution, pp. 127-132.

Palma, M.G. di (1978). Notizie sulle tartarughe maxine in Sicilia (Reptilia, Testudines). Il Naturalista Siciliano (4th ser.) 2(1-2): $1-6$.

Panou, A. and Moschonas, S. (1990). Incidental catches of Loggerhead turtles Caretta caretta in swordfish long lines in the Ionian Sea, Greece.

Pérès, J.M. (1985). History of the Mediterranean biota and the colonization of the depths. In: Margalef, M. (Ed.), Western Mediterranean. Key Environment Series, Pergamon Pres Ltd. Chapter 7, pp. 198-232. 
Pritchard, P., et al.; Bjorndal, K., and Balazs, G.H. (Eds.). (1983). Manual of sea turtle research and conservation techniques. Second edition. Centre for Environmental Education, Washington, D.C.

Ramsey, A.I.G. (1970). A search for the Green Turtles of the South Turkish coast, 1970 (with comment on Cyprus). Unpublished report submitted to the Fauna and Flora Preservation Society.

Raymond, P.W. (1984). Sea turtle hatchling disorientation and artificial beachfront lighting. Centre for Environmental Education, Washington, D.C.

Sarigül, G. (1989). Report to the Council of Europe Experts Meeting on marine turtles, 2-3 0ctober 1989, Strasbourg. 6pp.

Schleich, H.-H. (1984). Studies on the herpetology of Kouf National Park and adjacent areas of Cyrenaika. Final Report, Project 9044. IUCN, Gland, Switzerland.

Shabica, S.V. (1982). Planning for protection of sea turtle habitat. In, Bjorndal, K. (Ed.). Biology and Conservation of Sea Turtles. Smithsonian Institution, pp. 513-518.

Sutherland, J.M. (1984). Report on the biology and conservation of the Loggerhead Turtle Caretta caretta, from the Greek Island Zakynthos. Unpublished report.

Sutherland, J.M. (undated). Marine Turtle research project. Ionian Islands, Greece. 1987 season. Unpublished report.

Thibault, J.-C., Delaugerre, M., and Noblet, J.-F. (1984). Livre Rouge des Vertebrés menacés de la Corse. Parc naturel régional de la Corse, Ajaccio.

Van den Berk, V.M., Langeveld, M.J. and Sarigül, G. (1988). Observations of Cheloniidae and Trionychidae along the Cukurova coast, southern Turkey, Spring 1986 and 1987. In: Van der Have et al., (Eds.), South Turkey Project. A survey of waders and water $\overline{\text { fow } 1}$ in the Cukurova deltas, spring 1987. DHKD and WIWO, WIWO Report No. 22; Chapter 13, pp. 167-171.

Van Nierop, M.M., and Den Hartog, J.C. (1984). A study on the gut contents of five juvenile Loggerhead turtles, Caretta caret ta (Linnaeus) (Reptilia, Cheloniidae), from the south-eastern part of the North Atlantic 0cean, with emphasis on coelenterate identification. Zoologische Mededelingen 59(4): 35-54.

Venizelos, L. (1986). Guest editorial: Greek loggerheads face dangers. Marine Turtle Newsletter 39, p. 1.

Venizelos, L. (1987). Unpublished report prepared for 1st meeting of Group of Experts on Caretta caretta and Chelona mydas (Bern Convention), 1-2 September, Strasbourg.

Venizelos, L. (1988). Report released at Press Conference at the office of the Hellenic Society for the Protection of Nature, Athens, 12 July 1988.

Venizelos, L. (1989). Unpublished report. Zakynthos, 28 August 1989. 
Voesenek, L.A.C.J., and van Rooy, P.T.J.C. (1984). Herpetological Research on Eastern Sardinia: Proposal for a Biogenetic Reserve. Unpublished report to the Council of Europe. Research Institute for Nature Management, Arnhem; Dept. of Animal Ecology, Catholic University, Nijmegen; Societas Europaea Herpetologica.

Whitmore, C., Sarigül, G. and Yazgan, N. (in press, 1990). New legislation to protect sea turtle nesting areas in Turkey at Patara, the Göksu Delta and Ekinchik. Marine Turtle Newsletter.

Witham, R. (1982). Disruption of Sea Turtle habitat with emphasis on human influence. In, Bjorndal, K. (Ed.). Biology and Conservation of Sea Turtles, Smithsonian Institution. pp. 519-522.

Witham, R. (1985). Managing Florida (U.S.A.) Sea Turtle populations. Proceedings of the Symposium on Endangered Marine Animals and Marine Parks. Cochin, India. 12-16 January.

Worth, D.F., and Smith, J.B. (1976). Marine turtle nesting on Hutchinson Island, Florida, in 1973. Florida Marine Research Publication, 18: 1-17. 


\section{Sales agents for publications of the Council of Europe Agents de vente des publications du Conseil de l'Europe}

\author{
AUSTRALIA / AUSTRALIE \\ Hunter Publications \\ 58A, Gipps Street \\ AUS-3066 COLLINGWOOD, Victoria

\section{AUSTRIA / AUTRICHE} \\ Gerold und Co. \\ Graben 31 \\ A-1011 VIENNA 1 \\ BELGIUM / BELGIQUE \\ La Librairie européenne S.A. \\ 244 , rue de la Loi \\ B-1040 BRUSSELS
}

\section{CANADA}

Renouf Publishing Company Limited 1294 Algoma Road CDN-OTTAWA ONT KIB 3W8

\section{CYPRUS / CHYPRE}

\section{MAM}

The House of the Cyprus Book

P.O. Box 1722

CY-NICOSIA

\section{DENMARK / DANEMARK}

Munksgaard

Book and Subscription Service

P.O. Box 2148

DK-1016 COPENHAGEN K

\section{FINLAND / FINLANDE}

Akateeminen Kirjakauppa

Keskuskatu 1

P.O. Box 128

SF-00101 HELSINKI

\section{GERMANY / ALLEMAGNE}

Verlag Dr. Hans Heger

Herderstraße 56

Postfach 201363

D-5300 BONN

\section{GREECE / GRÈCE}

Librairie Kauffmann

28 , rue Stadiou

GR-ATHENS 132

\section{ICELAND / ISLANDE}

Bókabúd Máls og menningar

Laugavegi 18

IS-REYKJAVIK 101

\author{
IRELAND / IRLANDE \\ Government Stationery Office \\ Publications Section \\ Bishop Street \\ IRL-DUBLIN 8
}

ITALY / IT ALIE

Libreria Commissionaria Sansoni

Via Benedetto Fortini, 120/10

Casella Postale 552

I-50125 FLORENCE

\section{LUXEMBOURG}

Librairie Bourbon

(Imprimerie Saint-Paul)

11 , rue Bourbon

L-1249 LUXEMBOURG

\section{MALAYSIA / MALAISIE \\ Library Building \\ University of Malaya \\ P.O. Box 1127 \\ Jalan Pantai Baru \\ 59700 KUALA LUMPUR}

\section{NETHERLANDS / PAYS-BAS \\ InOr-publikaties \\ Noorderwal 38 \\ Postbus 14 \\ NL-7240 BA LOCHEM}

\section{NEW ZEALAND/}

NOUVELLE-ZÉLANDE

Government Printing Office

Mulgrave Street

(Private Bag)

NZ-WELLINGTON

\section{NORWAY / NORVÈGE}

Akademika, A/S Universitetsbokhandel P.O. Box 84

Blindern

$\mathrm{N}-0314$ OSLO

\section{PAKISTAN}

Tayyab M.S. Commercial Services

P.O. Box 16006

A-2/3, Usman Ghani Road

Manzoor Colony

PAK-KARACHI-44

\section{PORTUGAL}

Livraria Portugal

Rua do Carmo, 70

P-1200 LISBON
SPAIN / ESPAGNE

Mundi-Prensa Libros S.A.

Castelló 37

E-28001 MADRID

Libreria de la Generalitat

Rambla dels Estudis, 118

E-08002 BARCELONA

\section{SRI LANKA}

Centre for Curriculum Advancement 78 Eachamottai Road CL-JAFFNA

SWEDEN/SUÈDE

A ktiebolaget C.E. Fritzes

Regeringsgatan 12

Box 16356

S-10327 STOCKHOLM

SWITZERLAND / SUISSE

Buchhandlung Heinimann \& Co.

Kirchgasse 17

CH-8001 ZURICH

Librairie Payot

6 , rue Grenus

CH-1211 GENEVA 11

TAIWAN - HONG KONG SINGAPORE / SINGAPOUR

Mappamundi Taiwan

7 FI. 258, Nanking E. Rd.

Sec. 3 Taipei

TAIWAN R.O.C.

\section{TURKEY / TURQUIE}

Librairie Haset Kitapevi A.S. 469, Istiklâl Caddesi

Beyoglu

TR-IST ANBUL

\section{UNITED KINGDOM/ ROYAUME-UNI}

\section{HMSO}

Agency Section

51 Nine Elms Lane

GB-LONDON SW8 5DR

UNITED STATES and CANADA /

ÉTATS-UNIS et CANADA

Manhattan Publishing Company

1 Croton Point Avenue, P.O. Box 650

CROTON, N.Y. 10520

\section{STRASBOURG}

Mésange S.A.

Groupe Berger-Levrault

23, place Broglie

F-67081 STRASBOURG Cedex 


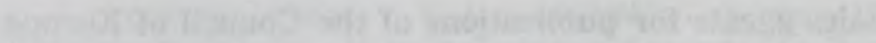

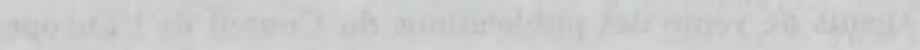

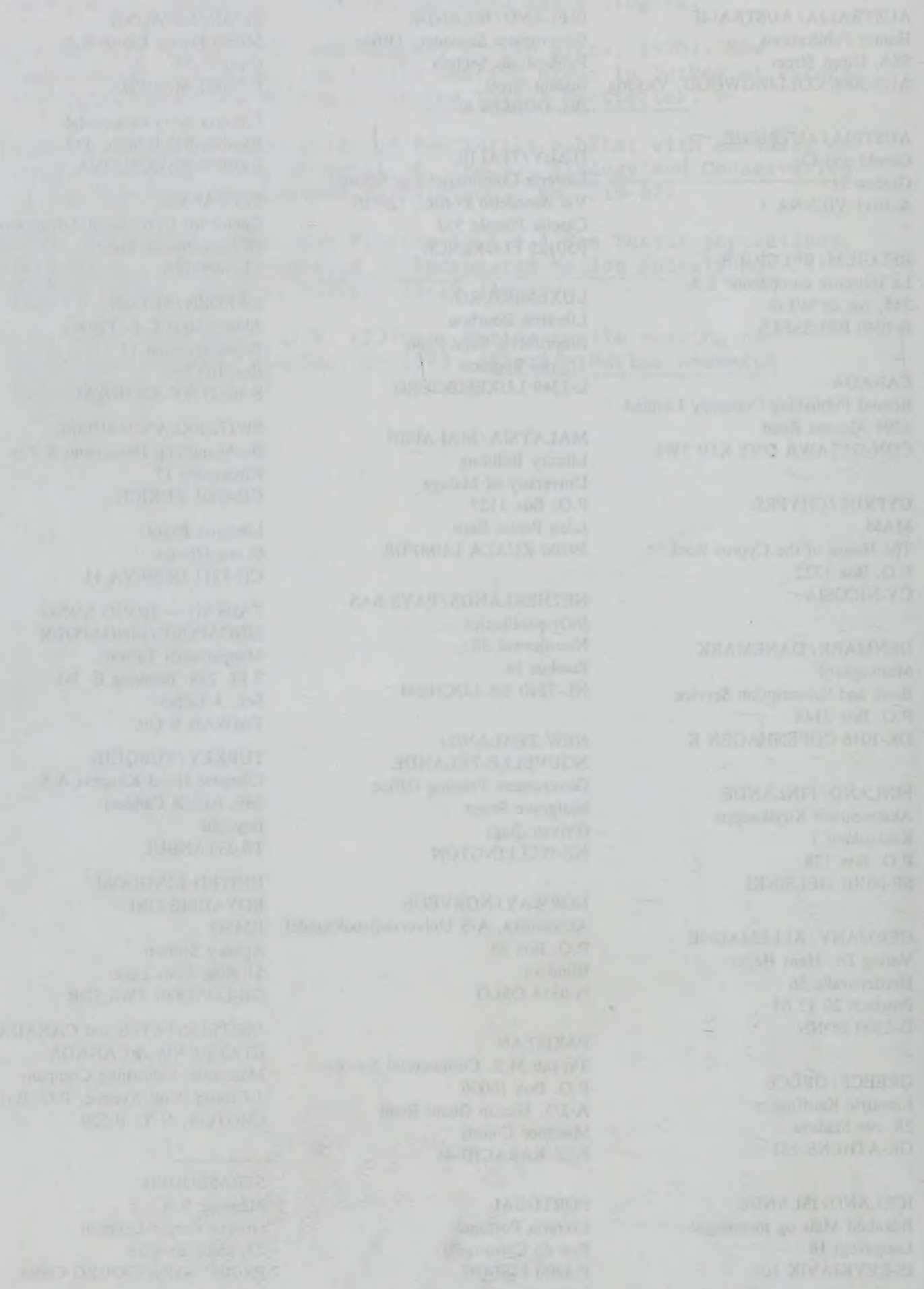



I 24372
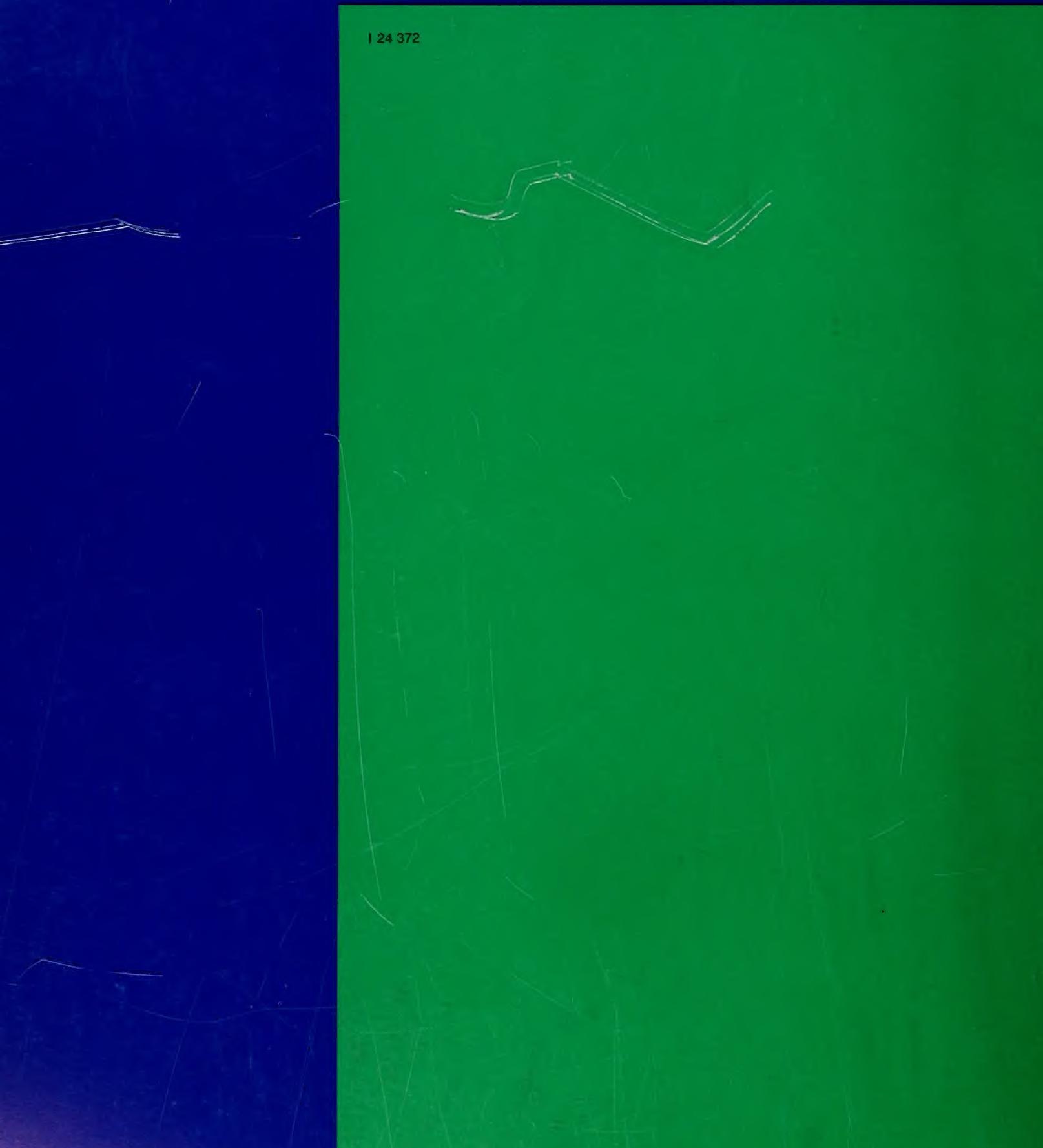

ISBN 92-871-1871-X 